$\operatorname{Argonne} \underset{\bigotimes}{\bigotimes}$

\title{
October 2007 Monitoring Results for Morrill, Kansas
}

\author{
Environmental Science Division
}




\begin{abstract}
About Argonne National Laboratory
Argonne is a U.S. Department of Energy laboratory managed by UChicago Argonne, LLC under contract DE-AC02-06CH11357. The Laboratory's main facility is outside Chicago, at 9700 South Cass Avenue, Argonne, Illinois 60439. For information about Argonne, see www.anl.gov.
\end{abstract}

\title{
Availability of This Report
}

This report is available, at no cost, at http://www.osti.gov/bridge. It is also available on paper to the U.S. Department of Energy and its contractors, for a processing fee, from:

U.S. Department of Energy

Office of Scientific and Technical Information

P.O. Box 62

Oak Ridge, TN 37831-0062

phone (865) 576-8401

fax (865) 576-5728

reports@adonis.osti.gov

\section{Disclaimer}

This report was prepared as an account of work sponsored by an agency of the United States Government. Reference herein to any specific commercial product, process, or service by trade name, trademark, manufacturer, or otherwise, does not necessarily constitute or imply its endorsement, recommendation, or favoring by the United States Government or any agency thereof. The views and opinions of document authors expressed herein do not necessarily state or reflect those of the United States Government or any agency thereof, Argonne National Laboratory, or UChicago Argonne, LLC. 


\section{October 2007 Monitoring Results for Morrill, Kansas}

by

Applied Geosciences and Environmental Management Section

Environmental Science Division, Argonne National Laboratory

March 2008

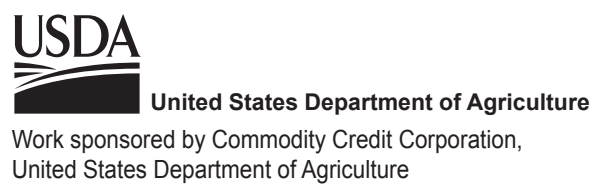




\section{Contents}

Notation.

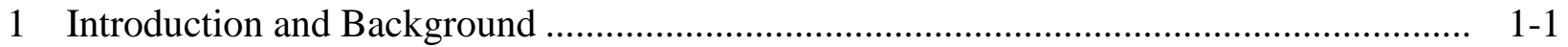

2 Sample Collection and Analysis Activities.......................................................... 2-1

$2.1 \quad$ Measurement of Groundwater Levels............................................................ $2-1$

2.2 Monitoring and Private Well Sampling and Analyses............................................ 2-1

2.3 Surface Water and Sediment Sampling and Analyses ........................................ 2-2

2.4 Vegetation Sampling and Analyses ........................................................................ 2-3

2.5 Handling and Disposal of Investigation-Derived Waste ...................................... 2-3

2.6 Quality Control for Sample Collection, Handling, and Analysis ........................... 2-4

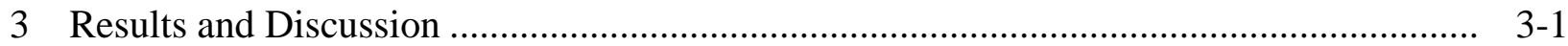

3.1 Groundwater Level Data............................................................................. $3-1$

3.2 Groundwater Analysis Results..................................................................... 3-2

3.2.1 Results of the Groundwater Analyses for VOCs ...................................... 3-2

3.2.2 Results of Groundwater Analyses for Indicators of Possible Contaminant

Degradation........................................................................................ 3-2

3.3 Surface Water and Sediment Analysis Results ............................................. 3-3

3.4 Vegetation Analysis Results ......................................................................... $3-3$

4 Conclusions and Recommendations ............................................................... $4-1$

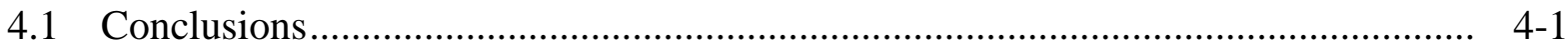

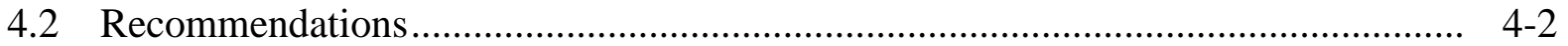

5 References

Appendix A: Sequence of Sampling Activities at Morrill, Kansas, in October 2007 ............ A-1

Appendix B: Data Summary for Verification VOCs Analyses by Envirosystems, Inc.......... B-1

\section{Figures}

$1.1 \quad$ Monitoring network at Morrill, as of October 2007 ........................................... 1-3

2.1 Locations of surface water and creek bed sediment sampling along Terrapin Creek at Morrill in October 2007 
2.2 Locations of native vegetation (tree branch and leaf tissue) sampling along Terrapin Creek at Morrill in July 2007

3.1a Potentiometric surface at Morrill, based on water levels measured manually on January 8, 2007

3.1b Potentiometric surface at Morrill, based on water levels measured manually on July 27, 2007

3.2a Hydrographs summarizing results of long-term water level monitoring in wells MW1S-MW4S and MW6S-MW8S at Morrill, from June 16, 2006 to January 8, 2007

3.2b Hydrographs summarizing results of long-term water level monitoring in wells MW1S-MW4S and MW6S-MW8S at Morrill, from January 1, 2007, to July 27, 2007

3.3 Carbon tetrachloride levels in groundwater at Morrill, October 2007.

3.4 Lateral extent of the carbon tetrachloride contamination in groundwater at Morrill, as interpreted on the basis of sampling and analysis in October 2007 and the flow direction determined on January 8, 2007, and July 27, 2007

4.1 Carbon tetrachloride levels in groundwater at Morrill in October 2003 and October 2007

\section{Tables}

3.1 Goundwater levels at Morrill, measured by hand on January 8, July 27, and October 1-3, 2007

3.2 Results of analyses at the AGEM Laboratory for volatile organic compounds in groundwater samples collected at Morrill, October 2003 to October 2007.

3.3 Field measurements for groundwater samples collected at Morrill, October 2003 to October 2007.

3.4 Results of attenuation parameter analyses for groundwater samples collected at Morrill, October 2003 to October 2007.

3.5 Results of analyses at the AGEM Laboratory for volatile organic compounds in surface water and sediment samples collected at Morrill, March 2007 to October 2007. 
3.6 Results of analyses at the AGEM Laboratory for volatile organic compounds in vegetation samples collected at Morrill in October 2006 to July 2007

A.1 Sequence of sampling activities at Morrill in October 2007................................ A-2 


\section{Notation}

AGEM Applied Geosciences and Environmental Management

AMSL above mean sea level

BGL below ground level

${ }^{\circ} \mathrm{C} \quad$ degree(s) Celsius

CCC Commodity Credit Corporation

COC chain of custody

EPA U.S. Environmental Protection Agency

$\mathrm{ft} \quad$ foot (feet)

gal gallon(s)

hr hour

in. inch(es)

KDHE Kansas Department of Health and Environment

$\mu \mathrm{g} / \mathrm{kg} \quad$ microgram(s) per kilogram

$\mu \mathrm{g} / \mathrm{L} \quad$ microgram(s) per liter

$\mu \mathrm{S} / \mathrm{cm} \quad$ microsiemen(s) per centimeter

$\mathrm{mg} / \mathrm{L} \quad$ milligram(s) per liter

$\mathrm{mV} \quad$ millivolt(s)

$\mathrm{nM}$ nanomolar

ORP oxidation-reduction potential

TOC top of casing

USDA U.S. Department of Agriculture

VOC volatile organic compound 


\section{October 2007 Monitoring Results for Morrill, Kansas}

\section{Introduction and Background}

In September 2005, the Commodity Credit Corporation of the U.S. Department of Agriculture (CCC/USDA) initiated periodic sampling of groundwater in the vicinity of a grain storage facility formerly operated by the CCC/USDA at Morrill, Kansas. On the basis of available information, the CCC/USDA believes that one or more third parties operated this facility after termination of the CCC/USDA's lease in 1971. The sampling at Morrill is being performed on behalf of the CCC/USDA by Argonne National Laboratory, in accord with a monitoring program approved by the Kansas Department of Health and Environment (KDHE), to monitor levels of carbon tetrachloride contamination identified in the groundwater at this site (Argonne 2004, 2005a). Under the KDHE-approved monitoring plan (Argonne 2005b), the groundwater has been sampled twice yearly for a recommended period of two years. The samples are analyzed for volatile organic compounds (VOCs), as well as for selected geochemical parameters to aid in the evaluation of possible natural contaminant degradation (reductive dechlorination) processes in the subsurface environment. The sampling is presently conducted in a network of 12 monitoring wells and 3 private wells (Figure 1.1), at locations approved by the KDHE. The scope of the originally approved monitoring has been expanded to include vegetation sampling (initiated in October 2006) and surface water and stream bed sediment sampling (initiated in March 2007).

The analytical results for groundwater sampling events at Morrill in September 2005, March 2006, September 2006, and March 2007 were documented previously (Argonne 2006a, $2007 \mathrm{c}, \mathrm{e})$. The results have demonstrated the presence of carbon tetrachloride contamination, at levels exceeding the KDHE Tier 2 Risk-Based Screening Level $(5.0 \mu \mathrm{g} / \mathrm{L})$ for this compound, in a groundwater plume extending generally south-southeastward from the former CCC/USDA facility, toward Terrapin Creek at the south edge of the town. Little clear pattern of change in the concentrations observed at the individual monitoring points and little plume migration have been observed in previous monitoring events. Low levels $(\leq 1.3 \mu \mathrm{g} / \mathrm{L})$ of carbon tetrachloride have persistently been detected at monitoring well MW8S, however, along an intermittent tributary to Terrapin Creek. This observation suggests a possible risk of contamination of the surface waters of the creek. In light of these findings, in 2006 the CCC/USDA recommended expansion of the approved monitoring program to include the collection and analysis of surface water samples along Terrapin Creek (Argonne 2007e). At the request of the KDHE (KDHE 2007a), locations for both surface water and shallow sediment sampling were discussed with the KDHE in January 
2007. An addendum to the existing monitoring plan and a standard operating procedure (SOP AGEM-15) for sediment sampling were submitted to the KDHE on the basis of these discussions (Argonne 2007a,b).

This report presents the results of groundwater, surface water, and sediment sampling performed at Morrill in October 2007, in accord with the monitoring plan (Argonne 2005b) and the addendum to that plan (Argonne 2007a). To supplement these studies, Argonne also sampled natural vegetation along Terrapin Creek in October 2006, April 2007, and July 2007 for analyses for VOCs. The results of the plant tissue analyses are included in this report.

The October 2007 groundwater sampling at Morrill represents the fifth and final monitoring event performed under the recommended two-year monitoring program approved by the KDHE. 


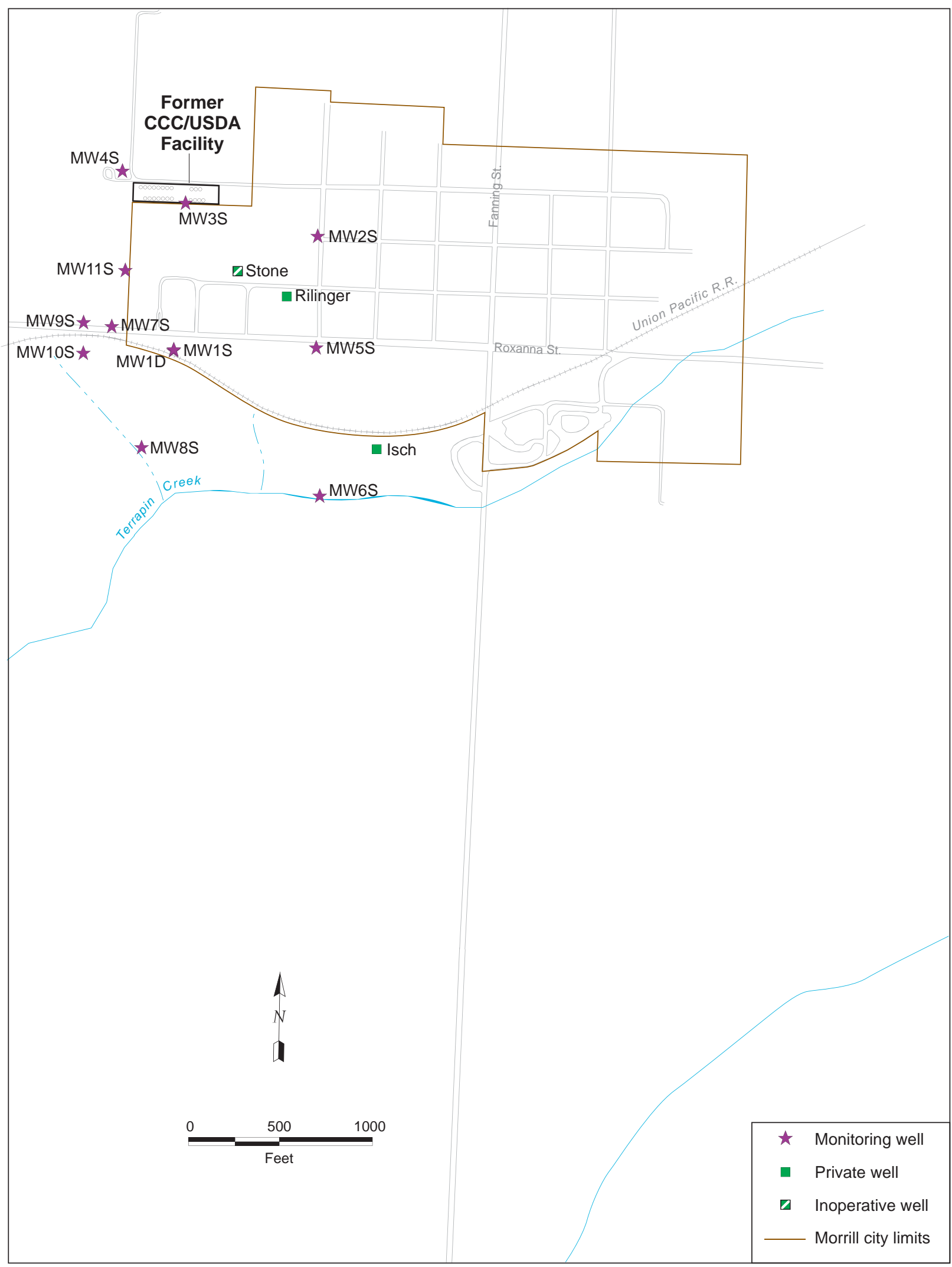

FIGURE 1.1 Monitoring network at Morrill, as of October 2007. 


\section{Sample Collection and Analysis Activities}

\subsection{Measurement of Groundwater Levels}

Manual water level measurements were made in conjunction with well sampling events. Before each well was purged in preparation for sampling, a water level indicator was used (where possible) to measure the depth to groundwater and the total depth from the top of the well casing, with an accuracy of $\pm 0.1 \mathrm{ft}$.

Monitoring wells MW1D and MW1S-MW11S and the Stone, Isch, and Rilinger private wells (Figure 1.1) were sampled on October 1-3, 2007. Monitoring well MW4S was resampled on January 11, 2008, because of a quality control concern related to the sample collected on October 3, 2007. The Rilinger well was resampled on October 8, 2007, and January 11, 2008, because of a concern regarding the representativeness of the sample collected on October 3 , 2007. (See Section 2.6 for further details.) Manual water level measurements were made each time a sample was collected.

Data recorders currently installed in wells MW1S-MW4S and MW6S-MW8S are gathering long-term data on the groundwater elevation and gradient at Morrill. The data recorders in these wells were downloaded on January 8, 2007, and on July 27, 2007. Water levels were measured manually in all monitoring wells on these dates, as well as in conjunction with the sampling event on October 1-3, 2007.

The groundwater level data are discussed in Section 3.1.

\subsection{Monitoring and Private Well Sampling and Analyses}

After measurement of water levels and dissolved oxygen concentrations, the wells were purged of a minimum of three well volumes. Field measurements of temperature, $\mathrm{pH}$, and conductivity were taken, while purging continued until the measurements stabilized. Field measurements of carbon dioxide, iron(II), and oxidation-reduction potential (ORP) were made for the evaluation of possible biodegradation processes, as outlined in the monitoring plan (Argonne 2005b). All sampling and field analyses were performed in accord with procedures in 
the Master Work Plan (Argonne 2002). The sequence of activities during the October well sampling event is summarized in Appendix A.

Groundwater samples for VOCs and selected geochemical analyses identified in the monitoring plan (Argonne 2005b) were collected in appropriate laboratory containers, labeled, packaged, and chilled to $4^{\circ} \mathrm{C}$ by placement in ice-filled coolers. The samples were shipped via an overnight delivery service to the Applied Geosciences and Environmental Management (AGEM) Laboratory at Argonne for VOCs analyses with U.S. Environmental Protection Agency (EPA) Method 524.2 (EPA 1995). Separate aliquots of selected samples (chosen in the field) were shipped to Envirosystems, Inc., Columbia, Maryland, for verification VOCs analyses.

Samples for laboratory analyses of degradation parameters were collected and shipped to TestAmerica Laboratories (formerly Severn-Trent Laboratories), South Burlington, Vermont. The analyses included dissolved chloride, sulfate, nitrate, and phosphate concentrations by EPA Method 300; total alkalinity by EPA Method 310.1; nitrate/nitrite nitrogen by EPA Method 353.2; sulfide by EPA Method 376.2; total organic carbon by EPA Method 415.1; and dissolved metals (aluminum, calcium, iron, magnesium, manganese, phosphorus, potassium, silicon, sodium, and zinc) by EPA Method 6010 (EPA 1998a). Analyses for the natural attenuation indicators methane, ethane, and ethene were conducted with Method RSK-175 (Kampbell and Vandegrift 1998). Prior sampling events included analyses for the attenuation parameters nitrite nitrogen (by EPA Method 354.1 at TestAmerica Laboratories) and dissolved hydrogen (by Method AM20GAX at Microseeps Laboratory, Pittsburgh, Pennsylvania).

The analytical results are presented and discussed in Section 3.2.

\subsection{Surface Water and Sediment Sampling and Analyses}

At the request of the KDHE (2007a), surface water samples and corresponding samples of the underlying shallow sediments in the creek bed were collected for VOCs analyses on October 8, 2007, at five locations along Terrapin Creek (Figure 2.1), as outlined in the addendum (Argonne 2007a) to the monitoring plan (Argonne 2005b). The sampling was conducted in accord with procedures in the Master Work Plan (Argonne 2002) and SOP AGEM-15 (Argonne 2007b). Surface water flow in Terrapin Creek south of Morrill originates at the outfall from an earthen dam and retention pond located approximately 1,900 ft southwest of the former 
CCC/USDA facility (Figure 2.1). Surface water and sediment sampling location SMB, which is directly downstream of this outfall, is believed to lie upgradient, or cross-gradient, to groundwater flow (and hence possible contaminant migration) from the vicinity of the former CCC/USDA facility. (See Section 3.1.) Sampling locations SM1-SM4 were selected to lie downgradient and downstream from the carbon tetrachloride detections previously identified at MW8S and elsewhere in the monitoring well network.

Samples of surface water were collected in appropriate containers, labeled, preserved at $4^{\circ} \mathrm{C}$, and shipped by an overnight delivery service to the AGEM Laboratory for VOCs analyses with EPA Method 524.2 (EPA 1995). Samples of the shallow creek bed sediments were collected by directly scooping the materials into appropriate laboratory containers (Argonne 2006b, 2007b). The samples were labeled, preserved on dry ice, and shipped to the AGEM Laboratory for sample preparation and VOCs analyses with modified EPA Methods 5030B and 8260B

The results of the analyses are presented and discussed in Section 3.3.

\subsection{Vegetation Sampling and Analyses}

Samples of natural vegetation, consisting of branch and leaf tissue material recovered from mature trees, were collected on July 26, 2007, at 18 locations along Terrapin Creek and its intermittent tributaries south and southeast of the former CCC/USDA facility (Figure 2.2). The sampling locations were selected to lie in the apparent direction of groundwater flow from the former facility. The tissue samples were collected in appropriate laboratory containers, labeled, preserved on dry ice, and shipped to the AGEM Laboratory for VOCs analyses by a headspace technique based on a modification of EPA Method 5021 (http://www.epa.gov/epahome/index/).

The analytical results for the vegetation samples are presented and discussed in Section 3.4.

\subsection{Handling and Disposal of Investigation-Derived Waste}

Purge water generated as potentially contaminated investigation-derived waste was containerized on-site. The accumulated purge water was sampled and analyzed by Pace 
Analytical Services, Inc., Lenexa, Kansas. Methods used were EPA Method 5030/8260 for VOCs, EPA Method 504.1 for ethylene dibromide (EDB), and EPA Method 300 for nitrate as nitrogen. Carbon tetrachloride was detected at $1.3 \mu \mathrm{g} / \mathrm{L}$, but EDB and other VOCs were not detected. Nitrate was present at $7.7 \mathrm{mg} / \mathrm{L}$. With the approval of KDHE, the water was disposed of at the Sabetha, Kansas, publicly owned treatment works on October 25, 2007 (Sabetha 2007).

\subsection{Quality Control for Sample Collection, Handling, and Analysis}

The quality control/quality assurance procedures followed during the October 2007 monitoring event are described in detail in the Master Work Plan (Argonne 2002) and SOP AGEM-15 (Argonne 2007b). These procedures are summarized as follows:

- Sample collection and handling activities were monitored by the documentation of samples as they were collected and the use of chain-ofcustody forms and custody seals to ensure sample integrity during handling and shipment.

- Samples designated for VOCs analyses were received with custody seals intact and at the appropriate preservation temperature. All samples were analyzed within the required holding times.

- Quality control samples (a field blank, an equipment rinsate, and trip blanks) were collected to monitor sample collection and handling activities. Method blanks were analyzed to monitor analytical methodologies. The detection of carbon tetrachloride in the rinsate sample indicated that the trace concentration of carbon tetrachloride (estimated at $0.5 \mu \mathrm{g} / \mathrm{L}$ ) detected in the initial sample from upgradient monitoring well MW4S resulted from incomplete equipment decontamination prior to collection of the sample. In subsequent resampling of MW04, carbon tetrachloride was not detected. Other quality control samples were free of carbon tetrachloride and chloroform contamination.

- The Rilinger well had been returned to service only a brief interval before it was sampled on October 3, 2007, upon reconnection to electrical power. This 
well had not previously been pumped for some time. On October 3, 2007, the sample was collected after the pump had been allowed to run for several minutes. The carbon tetrachloride value measured $(13 \mu \mathrm{g} / \mathrm{L})$ was surprisingly high in comparison to previous results (not detected to $2.6 \mu \mathrm{g} / \mathrm{L}$ in five sampling events from June 2004 to May 2007). The Rilinger well was resampled on October 8,2007, after the pump had been allowed to run for five minutes, with an estimated carbon tetrachloride concentration of $0.4 \mu \mathrm{g} / \mathrm{L}$. In an additional resampling on January 11, 2008, after the outside tap had run for ten minutes, a carbon tetrachloride concentration of $6.2 \mu \mathrm{g} / \mathrm{L}$ was measured. This last concentration is considered to be the most representative value for the Rilinger well during the reporting period; it is shown in the figures in this document.

- Groundwater samples were analyzed for VOCs at the AGEM Laboratory with the purge-and-trap method on a gas chromatograph-mass spectrometer system. Calibration checks with each sample delivery group were required to be within $\pm 20 \%$ of the standard. Surrogate standard determinations performed on samples and blanks were within the specified range of $80-120 \%$ for all samples, in either the initial analysis or a successful reanalysis.

- In accordance with the procedures defined in the Master Work Plan (Argonne 2002), the analyses of water samples at the AGEM Laboratory were verified at a second laboratory. Two groundwater samples were analyzed according to the EPA's Contract Laboratory Program methodology by Envirosystems, Inc. For the samples from monitoring wells MW1S and MW11S, lower carbon tetrachloride concentrations were reported by Envirosytems than by the AGEM Laboratory. In contrast, for the chloroform results, agreement between the two laboratories was good. Summary pages for the verification organic analyses are in Appendix B.

- Samples shipped to TestAmerica Laboratories for attenuation parameter analyses were received with custody seals intact and at the appropriate preservation conditions. All samples were analyzed within the required holding times. (Although the analyses of some samples for nitrate with EPA Method 300 did occur beyond the 48-hr holding time limit, the primary 
analyses of preserved sample volumes for total nitrogen with EPA Method 353.2 were within the holding time limit.) Results were within the acceptable limits for laboratory quality control samples prepared and analyzed with the samples to evaluate accuracy and precision. 


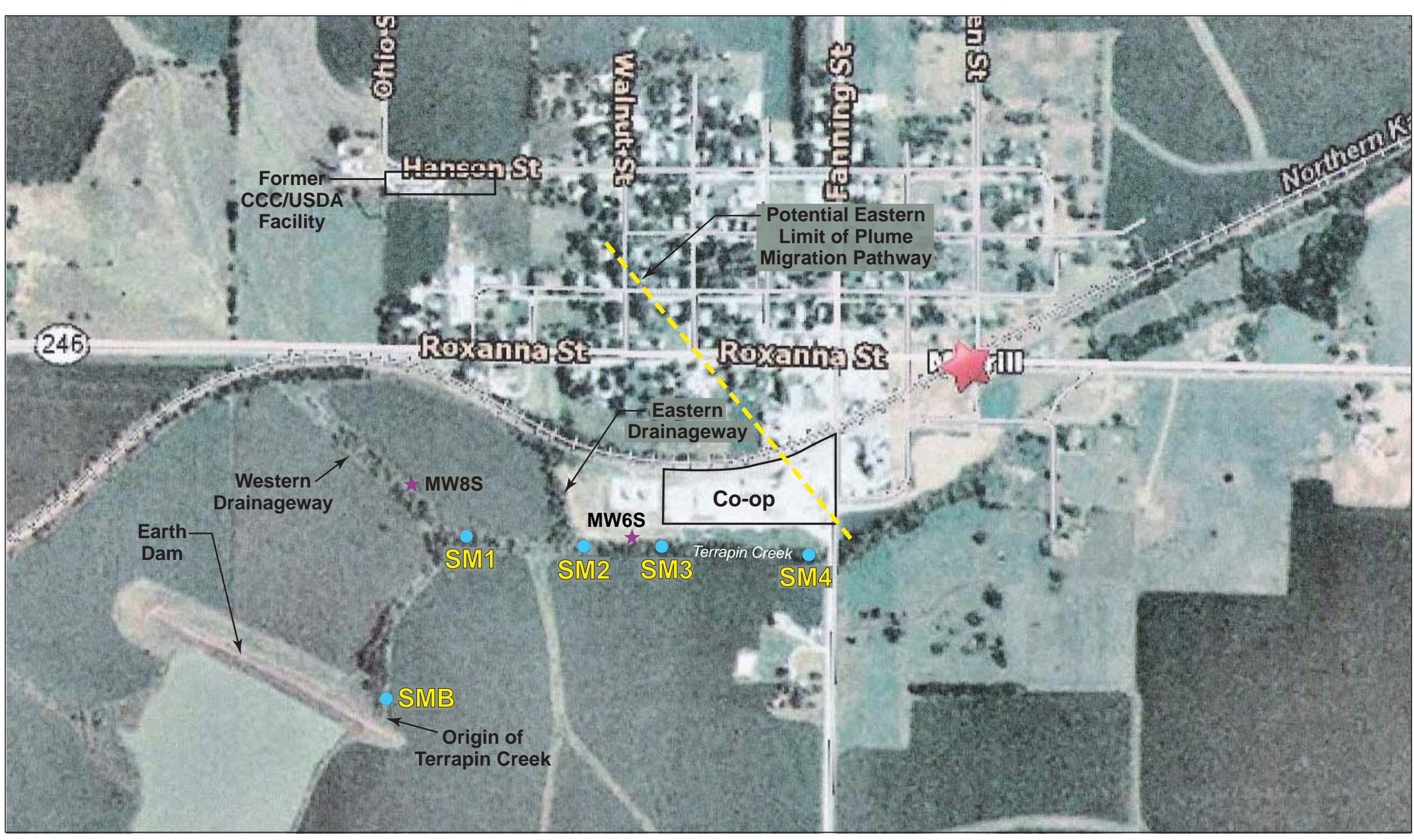

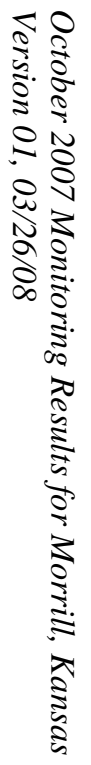

FIGURE 2.1 Locations of surface water and creek bed sediment sampling (SM1-SM4, SMB) along Terrapin Creek at Morrill in October 2007. 


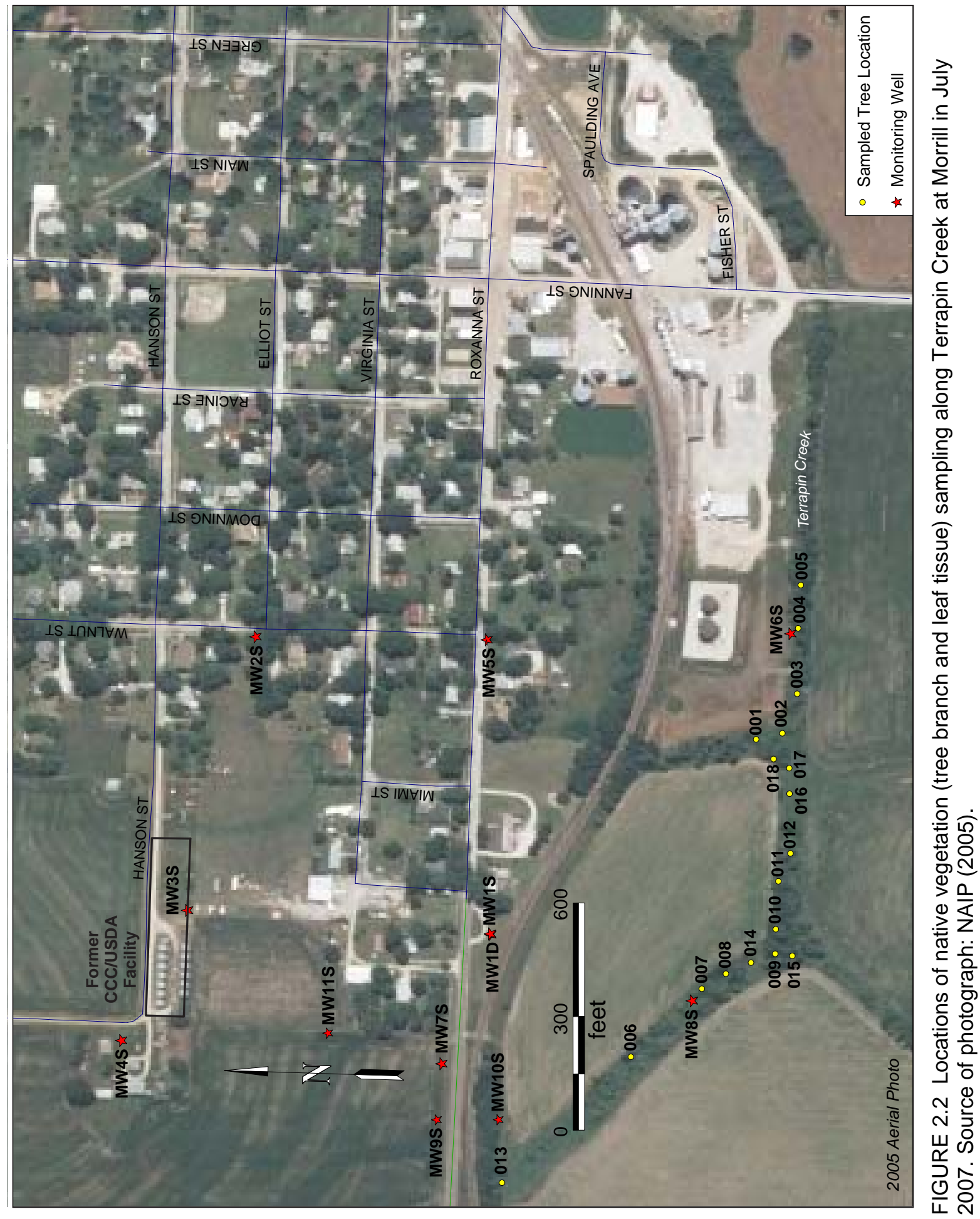




\section{Results and Discussion}

\subsection{Groundwater Level Data}

Depths to groundwater were measured manually in all available monitoring wells on January 8, 2007, July 27, 2007, and October 1-3, 2007 (during sampling). The hand-measured water level data are in Table 3.1. The tables are grouped at the end of the Section 3 text, before the figures.

The potentiometric surface at Morrill, based on manual measurements on January 8, 2007, and July 27, 2007, is depicted in Figures 3.1a,b, respectively. The recent results are consistent with previous measurements, indicating a groundwater flow direction toward the south-southeast from the former CCC/USDA facility. Persistently low water levels observed at MW11S (Figures 3.1a,b of this report and Figure 4.3 in Argonne 2007e) empirically suggest the apparent presence of a groundwater "sink" southwest of the former facility in the vicinity of this monitoring well.

Data for the long-term recording transducers installed in monitoring wells MW1S-MW4S and MW6S-MW8S for the periods (1) June 16, 2006, through January 8, 2007, and (2) January 1, 2007, through July 27, 2007, are summarized in Figures 3.2a,b, respectively. Groundwater levels at the site rose markedly in April-May 2007 and then returned to more typical levels. Responses to individual recharge events are most prominent in the traces for wells MW1S, MW3S, and MW7S. The traces for wells MW6S and MW8S show the least variation over time, in keeping with the shallow groundwater at these locations near Terrapin Creek.

The cause for the drawdown spikes apparent in the hydrograph for monitoring well MW8S in late July and August 2006 is unknown. Continued monitoring has not yet suggested an explanation. 


\subsection{Groundwater Analysis Results}

\subsubsection{Results of the Groundwater Analyses for VOCs}

The analytical data for VOCs in the groundwater samples collected in October 2007 are in Table 3.2, together with data for the previous sampling events conducted under the KDHEapproved monitoring plan (Argonne 2005b). The October 2007 data for carbon tetrachloride are illustrated in Figure 3.3.

Carbon tetrachloride was detected at 8 of the 15 monitoring locations, at concentrations ranging from $<1 \mu \mathrm{g} / \mathrm{L}$ (at MW8S) to a maximum of $61 \mu \mathrm{g} / \mathrm{L}$ at MW3S. Low levels of chloroform $(<1 \mu \mathrm{g} / \mathrm{L}$ to a maximum of $2.7 \mu \mathrm{g} / \mathrm{L}$ at MW1S) were detected in association with the carbon tetrachloride at 6 of the 8 locations (all locations except MW8S and the Stone well).

In comparison to the March 2007 sampling event, the present results indicate that carbon tetrachloride concentrations have increased somewhat at three locations (MW1S, MW11S, Rilinger). The concentrations were unchanged or decreased at the remaining sampling locations. Figure 3.4 indicates the position of the contaminant plume in October 2007. Table 3.2 demonstrates no consistent patterns of changing contaminant levels or migration since the CCC/USDA began its investigations at Morrill in 2003.

\subsubsection{Results of Groundwater Analyses for Indicators of Possible Contaminant Degradation}

The results of field measurements and selected laboratory geochemical analyses of the groundwater samples are summarized in Tables 3.3 and 3.4. The reported parameters can be used to estimate whether the in situ conditions at Morrill are suitable for possible degradation of carbon tetrachloride by natural anaerobic (reductive dechlorination) processes, as outlined in KDHE (2001) and EPA (1998b) guidance for the evaluation of these conditions.

In monitoring to date, no substantial evidence has been found for anaerobic biodegradation of carbon tetrachloride at Morrill. Though the data density and distribution of sampling points could affect this interpretation to an extent, the available results do not suggest that better sampling coverage would reveal a significant potential for anaerobic biodegradation of carbon tetrachloride at this site. 


\subsection{Surface Water and Sediment Analysis Results}

The results of VOCs analyses of the surface water and shallow sediment samples collected (at the request of the KDHE) along Terrapin Creek are in Table 3.5.

No carbon tetrachloride was detected in the surface water samples at an analytical method detection limit of $0.1 \mu \mathrm{g} / \mathrm{L}$. Similarly, no carbon tetrachloride was identified in the associated sediment samples at an analytical method detection limit of $1.0 \mu \mathrm{g} / \mathrm{kg}$. The October 2007 results therefore indicate that the surface waters and underlying sediments of Terrapin Creek have not been impacted by carbon tetrachloride contamination.

\subsection{Vegetation Analysis Results}

Experience gained by the CCC/USDA and Argonne in monitoring of the phytoremediation treatment programs in operation at the Murdock, Nebraska, former CCC/USDA grain storage facility and at the Argonne National Laboratory facility near Chicago, Illinois (Argonne 2005c, 2006b, 2007d; LaFreniere et al., 2006) has demonstrated that the detection of VOCs in native vegetation, and particularly in the branch tissues of trees, can provide a sensitive indicator of the presence of VOCs in the surface water or shallow groundwater to which these plants are exposed. This experience has also demonstrated, however, that the occurrence of VOCs contamination in branch tissues is associated with seasonal uptake of water by the trees during the active growing season; VOC levels in the plant tissues appear to fall rapidly as the trees enter their dormancy period during the fall and winter months.

At the request of the CCC/USDA, branch and leaf tissue samples from mature native trees were collected for VOCs analyses at 18 locations along Terrapin Creek and along several intermittent tributary drainages that feed into Terrapin Creek, in the area south and downgradient of the former CCC/USDA facility. The tissue samples were collected on July 26, 2007, at or near the peak of the growing season.

The analytical data for VOCs in the branch and leaf tissue samples are in Table 3.6. Trace concentrations of carbon tetrachloride were identified in branch tissue at two of the vegetation sampling locations (MR007 and MR014; Figure 2.2), at an analytical method detection limit of $0.1 \mu \mathrm{g} / \mathrm{kg}$. The observed results are qualitatively consistent with the absence of carbon tetrachloride in surface waters and shallow sediments discussed in Section 3.3, as well as with 
the nondetectable to trace concentrations of carbon tetrachloride identified in the shallow groundwater at monitoring well locations MW6S and MW8S (Section 3.2.1) along Terrapin Creek. Experience in the CCC/USDA's project at Murdock, Nebraska (Argonne 2007f), indicates that the mature trees along the creek banks would readily take up carbon tetrachloride if it was present in the water within reach of their root systems; the contaminant would then be observed in the tissue analyses. 
TABLE 3.1 Groundwater levels at Morrill, measured by hand on January 8, July 27, and October 1-3, 2007.

\begin{tabular}{|c|c|c|c|c|c|c|c|c|c|}
\hline \multirow[b]{3}{*}{ Well } & & & \multirow{3}{*}{$\begin{array}{l}\text { Top of Casing } \\
\text { Elevation }^{\mathrm{b}} \\
\text { (ft AMSL) }\end{array}$} & \multicolumn{2}{|c|}{ January 8, 2007} & \multicolumn{2}{|c|}{ July 27,2007} & \multicolumn{2}{|c|}{ October 1-3, 2007} \\
\hline & \multicolumn{2}{|c|}{ Horizontal Location $^{\mathrm{a}}$ (ft) } & & Depth to & Groundwater & Depth to & Groundwater & Depth to & Groundwater \\
\hline & Northing & Easting & & (ft BGL) & (ft AMSL) & (ft BGL) & (ft AMSL) & (ft BGL) & (ft AMSL) \\
\hline MW1S & 589130.20 & 1957316.76 & 1124.68 & 26.79 & 1097.89 & 22.20 & 1102.48 & 21.65 & 1103.03 \\
\hline MW1D & 589130.20 & 1957316.76 & 1124.63 & 26.43 & 1098.20 & 22.17 & 1102.46 & 22.75 & 1101.88 \\
\hline MW2S & 589789.61 & 1958063.43 & 1137.07 & 37.83 & 1099.24 & 30.50 & 1106.57 & 31.17 & 1105.90 \\
\hline MW3S & 589929.06 & 1957333.78 & 1135.76 & 31.73 & 1104.03 & 22.31 & 1113.45 & 22.70 & 1113.06 \\
\hline MW4S & 590083.24 & 1956982.15 & 1143.61 & 42.72 & 1100.89 & 30.18 & 1113.43 & 31.11 & 1112.50 \\
\hline MW5S & 589182.24 & 1958089.03 & 1122.21 & 26.50 & 1095.71 & 18.47 & 1103.74 & 19.55 & 1102.66 \\
\hline MW6S & 588385.33 & 1958149.44 & 1090.97 & 4.93 & 1086.04 & 4.77 & 1086.20 & 5.00 & 1085.97 \\
\hline MW7S & 589238.96 & 1956967.99 & 1119.86 & 20.40 & 1099.46 & 13.28 & 1106.58 & 12.40 & 1107.46 \\
\hline MW8S & 588590.43 & 1957169.82 & 1098.53 & 3.31 & 1095.22 & 3.06 & 1095.47 & 2.20 & 1096.33 \\
\hline MW9S & 589243.57 & 1956819.91 & 1118.31 & 19.29 & 1099.02 & 13.35 & 1104.96 & 14.00 & 1104.31 \\
\hline MW10S & 589081.48 & 1956829.05 & 1110.78 & 11.62 & 1099.16 & 6.32 & 1104.46 & 6.95 & 1103.83 \\
\hline MW11S & 589542.25 & 1957031.81 & 1133.08 & 36.45 & 1096.63 & 30.90 & 1102.18 & 31.55 & 1101.53 \\
\hline
\end{tabular}

a Horizontal coordinates are target location centers. Northings and eastings are Kansas State Plane Coordinates. Horizontal datum is North American Datum (NAD) 83.

b Vertical datum is National Geodetic Vertical Datum (NGVD) 88.

Source: Schwab-Eaton, Manhattan, Kansas. 
TABLE 3.2 Results of analyses at the AGEM Laboratory for volatile organic compounds in groundwater samples collected at Morrill, October 2003 to October 2007.

\begin{tabular}{|c|c|c|c|c|c|}
\hline \multirow[b]{2}{*}{ Location } & \multirow{2}{*}{$\begin{array}{l}\text { Screen } \\
\text { Interval } \\
\text { (ft BGL) }\end{array}$} & \multirow[b]{2}{*}{$\begin{array}{l}\text { Sample } \\
\text { Date }\end{array}$} & \multicolumn{3}{|c|}{ Concentration ( $\mu \mathrm{g} / \mathrm{L})$} \\
\hline & & & $\begin{array}{c}\text { Carbon } \\
\text { Tetrachloride }\end{array}$ & Chloroform & $\begin{array}{c}\text { Methylene } \\
\text { Chloride }\end{array}$ \\
\hline \multirow[t]{7}{*}{ MW1S } & $11-51$ & $10 / 23 / 03$ & 33 & 1.6 & $\mathrm{ND}^{\mathrm{a}}$ \\
\hline & & $6 / 2 / 04$ & 19 & $0.9 \mathrm{~J}^{\mathrm{b}}$ & ND \\
\hline & & 9/13/05 & 35 & 1.7 & ND \\
\hline & & $3 / 22 / 06$ & 40 & 1.8 & ND \\
\hline & & $9 / 20 / 06$ & 23 & $0.9 \mathrm{~J}$ & ND \\
\hline & & $3 / 21 / 07$ & 23 & 1.1 & ND \\
\hline & & $10 / 1 / 07$ & $56(56-37)$ & $2.7(2.7-1.8)$ & ND \\
\hline \multirow[t]{7}{*}{ MW1D } & $63-88$ & $10 / 22 / 03$ & ND & ND & ND \\
\hline & & $6 / 2 / 04$ & ND & ND & ND \\
\hline & & 9/13/05 & ND & ND & ND \\
\hline & & $3 / 19 / 06$ & ND & ND & $0.4 \mathrm{~J} \mathrm{~B}^{\mathrm{c}}$ \\
\hline & & $9 / 20 / 06$ & ND & ND & ND \\
\hline & & $3 / 21 / 07$ & ND & ND & ND \\
\hline & & $10 / 1 / 07$ & ND & ND & ND \\
\hline \multirow[t]{7}{*}{ MW2S } & $13-53$ & $10 / 22 / 03$ & ND & ND & ND \\
\hline & & $6 / 2 / 04$ & ND & ND & ND \\
\hline & & 9/14/05 & ND & ND & ND \\
\hline & & $3 / 21 / 06$ & ND & ND & ND \\
\hline & & 9/18/06 & ND & ND & ND \\
\hline & & $3 / 22 / 07$ & ND & ND & ND \\
\hline & & $10 / 3 / 07$ & ND & ND & ND \\
\hline \multirow[t]{7}{*}{ MW3S } & $18-48$ & $10 / 23 / 03$ & 89 & 2.7 & ND \\
\hline & & $6 / 2 / 04$ & 110 & 3.2 & ND \\
\hline & & 9/13/05 & 101 & 3.2 & ND \\
\hline & & $3 / 23 / 06$ & 91 & 2.6 & ND \\
\hline & & $9 / 20 / 06$ & 49 & 1.5 & ND \\
\hline & & $3 / 22 / 07$ & 84 & 2.3 & ND \\
\hline & & $10 / 3 / 07$ & 61 & 2.0 & ND \\
\hline \multirow[t]{8}{*}{ MW4S } & $17-47$ & $10 / 21 / 03$ & ND & ND & ND \\
\hline & & $6 / 4 / 04$ & ND & ND & ND \\
\hline & & 9/14/05 & ND & ND & ND \\
\hline & & $3 / 21 / 06$ & ND & ND & ND \\
\hline & & $9 / 18 / 06$ & ND & ND & ND \\
\hline & & $3 / 22 / 07$ & ND & ND & ND \\
\hline & & $10 / 3 / 07$ & $0.5 \mathrm{~J} \mathrm{R}^{\mathrm{d}}$ & ND & ND \\
\hline & & $1 / 11 / 08$ & ND & ND & ND \\
\hline \multirow[t]{7}{*}{ MW5S } & $15-55$ & $10 / 22 / 03$ & 5.8 & ND & ND \\
\hline & & $6 / 2 / 04$ & 7.0 & ND & ND \\
\hline & & 9/13/05 & 6.3 & $0.2 \mathrm{~J}$ & ND \\
\hline & & 3/22/06 & 7.3 & $0.2 \mathrm{~J}$ & ND \\
\hline & & $9 / 20 / 06$ & 6.4 & $0.3 \mathrm{~J}$ & ND \\
\hline & & $3 / 22 / 07$ & 6.5 & $0.4 \mathrm{~J}$ & ND \\
\hline & & $10 / 3 / 07$ & 4.0 & $0.3 \mathrm{~J}$ & ND \\
\hline
\end{tabular}


TABLE 3.2 (Cont.)

\begin{tabular}{|c|c|c|c|c|c|}
\hline \multirow[b]{2}{*}{ Location } & \multirow{2}{*}{$\begin{array}{l}\text { Screen } \\
\text { Interval } \\
\text { (ft BGL) }\end{array}$} & \multirow[b]{2}{*}{$\begin{array}{l}\text { Sample } \\
\text { Date }\end{array}$} & \multicolumn{3}{|c|}{ Concentration $(\mu \mathrm{g} / \mathrm{L})$} \\
\hline & & & $\begin{array}{c}\text { Carbon } \\
\text { Tetrachloride }\end{array}$ & Chloroform & $\begin{array}{c}\text { Methylene } \\
\text { Chloride }\end{array}$ \\
\hline \multirow[t]{6}{*}{ MW6S } & $10-25$ & $6 / 3 / 04$ & ND & ND & ND \\
\hline & & $9 / 14 / 05$ & ND & ND & ND \\
\hline & & $3 / 20 / 06$ & ND & ND & ND \\
\hline & & 9/18/06 & ND & ND & ND \\
\hline & & $3 / 21 / 07$ & ND & ND & ND \\
\hline & & $10 / 2 / 07$ & ND & ND & ND \\
\hline \multirow[t]{6}{*}{ MW7S } & $20-45$ & $6 / 3 / 04$ & 18 & ND & ND \\
\hline & & 9/12/05 & 43 & 1.1 & ND \\
\hline & & 3/22/06 & 21 & $0.4 \mathrm{~J}$ & ND \\
\hline & & 9/19/06 & 38 & $0.7 \mathrm{~J}$ & ND \\
\hline & & 3/20/07 & 16 & $0.4 \mathrm{~J}$ & ND \\
\hline & & $10 / 1 / 07$ & 8.1 & $0.2 \mathrm{~J}$ & ND \\
\hline \multirow[t]{6}{*}{ MW8S } & $10-25$ & $6 / 3 / 04$ & ND & ND & ND \\
\hline & & $9 / 14 / 05$ & $0.9 \mathrm{~J}$ & ND & ND \\
\hline & & 3/20/06 & $0.6 \mathrm{~J}$ & ND & $0.4 \mathrm{~J} \mathrm{~B}$ \\
\hline & & $9 / 19 / 06$ & 1.3 & ND & ND \\
\hline & & 3/20/07 & $0.6 \mathrm{~J}$ & ND & ND \\
\hline & & $10 / 2 / 07$ & $0.8 \mathrm{~J}$ & ND & ND \\
\hline \multirow[t]{4}{*}{ MW9S } & $38.83-53.83$ & 3/22/06 & ND & ND & ND \\
\hline & & $9 / 19 / 06$ & ND & ND & ND \\
\hline & & $3 / 20 / 07$ & ND & ND & ND \\
\hline & & $10 / 1 / 07$ & ND & ND & ND \\
\hline \multirow[t]{4}{*}{ MW10S } & $30-45$ & $3 / 21 / 06$ & ND & ND & ND \\
\hline & & $9 / 18 / 06$ & ND & ND & ND \\
\hline & & $3 / 21 / 07$ & ND & ND & ND \\
\hline & & $10 / 1 / 07$ & ND & ND & ND \\
\hline \multirow[t]{4}{*}{ MW11S } & $53-68$ & 3/22/06 & 39 & $0.9 \mathrm{~J}$ & ND \\
\hline & & 9/19/06 & 53 & 1.0 & ND \\
\hline & & 3/20/07 & 37 & $0.8 \mathrm{~J}$ & ND \\
\hline & & $10 / 1 / 07$ & 54 (54-29) & $1.2(1.2-0.6 \mathrm{~J})$ & ND \\
\hline \multirow[t]{6}{*}{ Isch } & Unk & 2/19/04 & ND & ND & ND \\
\hline & & $9 / 14 / 05$ & ND & ND & ND \\
\hline & & $3 / 23 / 06$ & ND & ND & ND \\
\hline & & 9/19/06 & ND & ND & ND \\
\hline & & 3/22/07 & ND & ND & ND \\
\hline & & $10 / 3 / 07$ & ND & ND & ND \\
\hline \multirow[t]{8}{*}{ Rillinger } & Unk & $6 / 4 / 04$ & ND & ND & ND \\
\hline & & 9/14/05 & 2.6 & $0.1 \mathrm{~J}$ & ND \\
\hline & & $3 / 19 / 06$ & ND & ND & $0.4 \mathrm{~J} \mathrm{~B}$ \\
\hline & & $9 / 19 / 06$ & ND & ND & ND \\
\hline & & 3/29/07 & 1.3 & 1.1 & ND \\
\hline & & $10 / 3 / 07^{e}$ & 13 & $0.4 \mathrm{~J}$ & ND \\
\hline & & $10 / 8 / 07$ & $0.4 \mathrm{~J}$ & ND & ND \\
\hline & & $1 / 11 / 08$ & 6.2 & $0.5 \mathrm{~J}$ & ND \\
\hline
\end{tabular}


TABLE 3.2 (Cont.)

\begin{tabular}{|c|c|c|c|c|c|}
\hline \multirow[b]{2}{*}{ Location } & \multirow{2}{*}{$\begin{array}{l}\text { Screen } \\
\text { Interval } \\
\text { (ft BGL) }\end{array}$} & \multirow[b]{2}{*}{$\begin{array}{l}\text { Sample } \\
\text { Date }\end{array}$} & \multicolumn{3}{|c|}{ Concentration ( $\mu \mathrm{g} / \mathrm{L})$} \\
\hline & & & $\begin{array}{c}\text { Carbon } \\
\text { Tetrachloride }\end{array}$ & Chloroform & $\begin{array}{c}\text { Methylene } \\
\text { Chloride }\end{array}$ \\
\hline \multirow[t]{6}{*}{ Stone } & $43^{f}$ & 6/4/04 & 10 & ND & ND \\
\hline & & $9 / 14 / 05$ & 2.6 & $0.3 \mathrm{~J}$ & ND \\
\hline & & $3 / 19 / 06$ & 14 & $0.8 \mathrm{~J}$ & $0.4 \mathrm{~J} \mathrm{~B}$ \\
\hline & & $9 / 19 / 06$ & 2.1 & ND & ND \\
\hline & & $3 / 22 / 07$ & 5.4 & $0.3 \mathrm{~J}$ & ND \\
\hline & & $10 / 3 / 07$ & 2.8 & ND & ND \\
\hline
\end{tabular}

a ND, not detected at instrument detection limit of $0.1 \mu \mathrm{g} / \mathrm{L}$.

b Qualifier J indicates an estimated concentration below the method quantitation limit of $1.0 \mu \mathrm{g} / \mathrm{L}$.

c Qualifier B indicates that the contaminant was present in the associated method blank.

d Qualifier R indicates that the contaminant was present in the associated equipment rinsate. Resampling confirmed that the well was free of contamination.

e Value considered non-representative because of recent reactivation of well. Well resampled on October 8, 2007, and January 11, 2008.

f Total depth. 
TABLE 3.3 Field measurements for groundwater samples collected at Morrill, October 2003 to October 2007.

\begin{tabular}{|c|c|c|c|c|c|c|c|c|c|}
\hline \multirow[b]{2}{*}{ Location } & \multirow{2}{*}{$\begin{array}{l}\text { Screen } \\
\text { Interval } \\
\text { (ft BGL) }\end{array}$} & \multirow[b]{2}{*}{$\begin{array}{l}\text { Sample } \\
\text { Date }\end{array}$} & \multirow[b]{2}{*}{$\begin{array}{c}\text { Temperature } \\
\left({ }^{\circ} \mathrm{C}\right)\end{array}$} & \multirow[b]{2}{*}{$\mathrm{pH}$} & \multicolumn{4}{|c|}{ Concentration $(\mathrm{mg} / \mathrm{L})$} & \multirow[b]{2}{*}{$\begin{array}{l}\text { ORP } \\
(\mathrm{mV})\end{array}$} \\
\hline & & & & & $\begin{array}{l}\text { Conductivity } \\
(\mu \mathrm{S} / \mathrm{cm})\end{array}$ & $\begin{array}{l}\text { Dissolved } \\
\text { Oxygen }\end{array}$ & $\begin{array}{l}\text { Carbon } \\
\text { Dioxide }\end{array}$ & Iron(II) & \\
\hline \multirow[t]{7}{*}{ MW1S } & $11-51$ & $10 / 23 / 03$ & 14.6 & 7.14 & 933 & $\_a$ & - & - & 13 \\
\hline & & 6/2/04 & 14.4 & 7.16 & 970 & - & - & - & - \\
\hline & & 9/13/05 & 15.3 & 6.95 & 1174 & 7.17 & 55 & 0 & 200 \\
\hline & & $3 / 22 / 06$ & 15.5 & 7.23 & 927 & 9.94 & 40 & 0.01 & 220 \\
\hline & & $9 / 20 / 06$ & 15.7 & 7.12 & 973 & 7.52 & 40 & 0.03 & NR \\
\hline & & $3 / 21 / 07$ & 16.6 & 6.48 & 960 & 5.45 & 40 & 0 & 88 \\
\hline & & $10 / 1 / 07$ & 16.0 & 6.80 & 886 & 6.79 & 30 & 0 & 128 \\
\hline \multirow[t]{7}{*}{ MW1D } & $63-88$ & 10/22/03 & 14.9 & 6.87 & 2620 & - & - & - & 25 \\
\hline & & $6 / 2 / 04$ & 13.9 & 6.87 & 2460 & - & - & - & - \\
\hline & & 9/13/05 & 15.5 & 6.56 & 2470 & - & - & - & - \\
\hline & & $3 / 19 / 06$ & 12.9 & 6.95 & 2460 & 5.11 & - & 0 & 230 \\
\hline & & 9/20/06 & 12.5 & 6.93 & 2690 & - & - & - & - \\
\hline & & $3 / 21 / 07$ & 15.3 & 6.39 & 2540 & 0.08 & 40 & 0.39 & 12 \\
\hline & & $10 / 1 / 07$ & 16.3 & 6.60 & 2230 & 6.79 & 45 & 0.44 & 5 \\
\hline \multirow[t]{7}{*}{ MW2S } & $13-53$ & 10/22/03 & 16.2 & 6.86 & 875 & - & - & - & 20 \\
\hline & & 6/2/04 & 16.9 & 7.07 & 861 & - & - & - & - \\
\hline & & 9/14/05 & 15.2 & 6.94 & 801 & 7.85 & 65 & - & 142 \\
\hline & & $3 / 21 / 06$ & 13.0 & 7.07 & 863 & 9.40 & 25 & 0.14 & 262 \\
\hline & & $9 / 18 / 06$ & 13.6 & 6.99 & 844 & 6.81 & 80 & 0 & 69 \\
\hline & & $3 / 22 / 07$ & 15.2 & 6.40 & 790 & 5.82 & 30 & 0 & 69 \\
\hline & & $10 / 3 / 07$ & 16.8 & 6.97 & 703 & 6.70 & 30 & 0.01 & 269 \\
\hline \multirow[t]{7}{*}{ MW3S } & $18-48$ & $10 / 23 / 03$ & 16.8 & 7.23 & 655 & - & - & - & 6 \\
\hline & & $6 / 2 / 04$ & 14.2 & 7.23 & 664 & - & - & - & - \\
\hline & & 9/13/05 & 14.6 & 7.13 & 663 & 8.82 & 100 & 0 & 223 \\
\hline & & $3 / 23 / 06$ & 8.9 & 7.16 & 662 & 6.74 & 25 & 0.08 & 269 \\
\hline & & 9/20/06 & 12.9 & 7.15 & 669 & 7.64 & - & 0 & 105 \\
\hline & & $3 / 22 / 07$ & 15.0 & 6.44 & 578 & 5.90 & 30 & 0.17 & 261 \\
\hline & & 10/3/07 & 15.3 & 6.97 & 594 & 0.38 & 20 & 0 & 282 \\
\hline \multirow[t]{8}{*}{ MW4S } & $17-47$ & $10 / 21 / 03$ & NR & 7.17 & 758 & - & - & - & - \\
\hline & & $6 / 4 / 04$ & 15.4 & 6.93 & 769 & - & - & - & - \\
\hline & & 9/14/05 & 15.4 & 7.30 & 751 & 8.00 & 50 & 0 & 174 \\
\hline & & $3 / 21 / 06$ & 6.7 & 7.25 & 729 & 10.9 & 25 & 0 & 154 \\
\hline & & $9 / 18 / 06$ & 13.1 & 7.25 & 728 & 8.05 & 50 & 0 & 41 \\
\hline & & $3 / 22 / 07$ & 14.2 & 6.53 & 765 & 5.91 & 25 & 0.10 & 78 \\
\hline & & $10 / 3 / 07$ & 16.4 & 6.95 & 715 & 7.40 & 30 & 0.10 & 281 \\
\hline & & $1 / 11 / 08$ & 11.3 & 7.56 & 751 & - & - & - & - \\
\hline \multirow[t]{7}{*}{ MW5S } & $15-55$ & 10/22/03 & 15.3 & 7.10 & 816 & - & - & - & 6 \\
\hline & & $6 / 2 / 04$ & 14.3 & 7.21 & 817 & - & - & - & - \\
\hline & & 9/13/05 & 16.0 & 7.04 & 763 & 13.9 & 60 & 0 & 228 \\
\hline & & $3 / 22 / 06$ & 13.9 & 7.25 & 781 & 4.52 & 35 & 0.06 & 234 \\
\hline & & $9 / 20 / 06$ & 13.9 & 7.19 & 787 & 5.82 & 35 & 0 & 73 \\
\hline & & 3/22/07 & 15.5 & 6.50 & 436 & 3.98 & 30 & 0.08 & 159 \\
\hline & & $10 / 3 / 07$ & 16.5 & 7.18 & 850 & 1.87 & 25 & 0.04 & 268 \\
\hline
\end{tabular}


TABLE 3.3 (Cont.)

\begin{tabular}{|c|c|c|c|c|c|c|c|c|c|}
\hline \multirow[b]{2}{*}{ Location } & \multirow{2}{*}{$\begin{array}{l}\text { Screen } \\
\text { Interval } \\
\text { (ft BGL) }\end{array}$} & \multirow[b]{2}{*}{$\begin{array}{l}\text { Sample } \\
\text { Date }\end{array}$} & \multirow[b]{2}{*}{$\begin{array}{c}\text { Temperature } \\
\left({ }^{\circ} \mathrm{C}\right)\end{array}$} & \multirow[b]{2}{*}{$\mathrm{pH}$} & \multicolumn{4}{|c|}{ Concentration $(\mathrm{mg} / \mathrm{L})$} & \multirow[b]{2}{*}{$\begin{array}{l}\text { ORP } \\
(\mathrm{mV})\end{array}$} \\
\hline & & & & & $\begin{array}{l}\text { Conductivity } \\
(\mu \mathrm{S} / \mathrm{cm})\end{array}$ & $\begin{array}{c}\text { Dissolved } \\
\text { Oxygen }\end{array}$ & $\begin{array}{l}\text { Carbon } \\
\text { Dioxide }\end{array}$ & Iron(II) & \\
\hline \multirow[t]{6}{*}{ MW6S } & $10-25$ & $6 / 3 / 04$ & 15.1 & 6.89 & 2410 & - & - & - & - \\
\hline & & $9 / 14 / 05$ & 14.1 & 7.06 & 2350 & 0.01 & 60 & 0 & 54 \\
\hline & & $3 / 20 / 06$ & 9.8 & 6.91 & 2360 & 1.37 & 60 & 0.38 & 89 \\
\hline & & $9 / 18 / 06$ & 12.5 & 6.96 & 2410 & 0.08 & 85 & 0.35 & -29 \\
\hline & & $3 / 21 / 07$ & 18.0 & 6.34 & 2450 & 0.12 & 40 & 0.78 & 75 \\
\hline & & $10 / 2 / 07$ & 17.1 & 7.33 & 2280 & 0.20 & 35 & 0.19 & 61 \\
\hline \multirow{6}{*}{ MW7S } & $20-45$ & $6 / 3 / 04$ & 13.8 & 7.19 & 763 & - & - & - & - \\
\hline & & $9 / 12 / 05$ & 15.0 & 7.26 & 760 & 8.35 & 50 & 0 & 240 \\
\hline & & $3 / 22 / 06$ & 15.2 & 7.32 & 740 & 5.52 & 25 & 0.03 & 268 \\
\hline & & 9/19/06 & 13.2 & 7.15 & 764 & 7.37 & 25 & 0 & 114 \\
\hline & & $3 / 20 / 07$ & 14.6 & 6.43 & 750 & 5.31 & 30 & 0 & 95 \\
\hline & & $10 / 1 / 07$ & 15.6 & 6.99 & 725 & 7.76 & 35 & 0.01 & 269 \\
\hline \multirow[t]{6}{*}{ MW8S } & $10-25$ & $6 / 3 / 04$ & 12.8 & 7.12 & 941 & - & - & - & - \\
\hline & & $9 / 14 / 05$ & 14.1 & 7.30 & 853 & 0.02 & 40 & 0 & 65 \\
\hline & & $3 / 20 / 06$ & 12.5 & 7.04 & 954 & 0.90 & 30 & 0.05 & 153 \\
\hline & & 9/19/06 & 11.8 & 7.09 & 903 & 0.58 & 50 & 0.13 & 284 \\
\hline & & $3 / 20 / 07$ & 11.0 & 6.52 & 1026 & 0.77 & 30 & 0 & 76 \\
\hline & & $10 / 2 / 07$ & 15.2 & 6.76 & 607 & 2.66 & 25 & 0.02 & 209 \\
\hline \multirow[t]{4}{*}{ MW9S } & $38.83-53.88$ & $3 / 22 / 06$ & 14.6 & 7.17 & 715 & 0.41 & 35 & 0 & 25 \\
\hline & & $9 / 19 / 06$ & 13.0 & 7.08 & 707 & 0.10 & 55 & 0 & 113 \\
\hline & & $3 / 20 / 07$ & 14.2 & 6.39 & 714 & 0.21 & 20 & 0 & 40 \\
\hline & & $10 / 1 / 07$ & 15.5 & 7.05 & 664 & 5.50 & 30 & 0 & 191 \\
\hline \multirow[t]{4}{*}{ MW10S } & $30-45$ & $3 / 21 / 06$ & 6.3 & 7.11 & 701 & 2.10 & 40 & 0.01 & 88 \\
\hline & & $9 / 18 / 06$ & 14.3 & 7.17 & 701 & 0.04 & 60 & 0.08 & 24 \\
\hline & & $3 / 21 / 07$ & 14.5 & 6.51 & 720 & 0.88 & 30 & 0 & 11 \\
\hline & & $10 / 1 / 07$ & 16.3 & 6.97 & 664 & 0.35 & 35 & 0.04 & 248 \\
\hline \multirow[t]{4}{*}{ MW11S } & 53-68 & $3 / 22 / 06$ & 14.8 & 7.33 & 762 & 9.40 & 30 & 0.06 & 237 \\
\hline & & $9 / 19 / 06$ & 13.0 & 7.24 & 764 & 1.42 & 30 & 0.02 & 158 \\
\hline & & $3 / 20 / 07$ & 14.6 & 6.33 & 782 & 3.90 & 30 & 0 & 76 \\
\hline & & $10 / 1 / 07$ & 16.4 & 6.49 & 624 & 6.57 & 35 & 0.04 & 241 \\
\hline \multirow[t]{6}{*}{ Isch } & Unk & $2 / 19 / 04$ & - & - & - & - & - & - & - \\
\hline & & $9 / 14 / 05$ & 20.4 & 6.73 & 2300 & - & - & - & - \\
\hline & & $3 / 23 / 06$ & 13.0 & 7.23 & 9400 & - & - & - & - \\
\hline & & $9 / 19 / 06$ & - & - & - & - & - & - & - \\
\hline & & $3 / 22 / 07$ & - & - & - & - & - & - & - \\
\hline & & $10 / 3 / 07$ & - & - & - & - & - & - & - \\
\hline \multirow[t]{7}{*}{ Rillinger } & Unk & $6 / 4 / 04$ & 15.9 & 6.99 & 2450 & - & - & - & - \\
\hline & & $9 / 14 / 05$ & - & - & - & - & - & - & - \\
\hline & & $3 / 19 / 06$ & 11.9 & 7.05 & 2550 & - & - & - & - \\
\hline & & $9 / 19 / 06$ & - & - & - & - & - & - & - \\
\hline & & $3 / 29 / 07$ & - & - & - & - & - & - & - \\
\hline & & $10 / 3 / 07$ & - & - & - & - & - & - & - \\
\hline & & $1 / 11 / 08$ & 12.2 & 7.46 & 884 & - & - & - & - \\
\hline
\end{tabular}


Version 00, 01/25/08

TABLE 3.3 (Cont.)

\begin{tabular}{|c|c|c|c|c|c|c|c|c|c|}
\hline \multirow[b]{2}{*}{ Location } & \multirow{2}{*}{$\begin{array}{l}\text { Screen } \\
\text { Interval } \\
\text { (ft BGL) }\end{array}$} & \multirow[b]{2}{*}{$\begin{array}{l}\text { Sample } \\
\text { Date }\end{array}$} & \multirow[b]{2}{*}{$\begin{array}{c}\text { Temperature } \\
\left({ }^{\circ} \mathrm{C}\right)\end{array}$} & \multirow[b]{2}{*}{$\mathrm{pH}$} & \multicolumn{4}{|c|}{ Concentration (mg/L) } & \multirow[b]{2}{*}{$\begin{array}{l}\text { ORF } \\
(\mathrm{mV}\end{array}$} \\
\hline & & & & & $\begin{array}{c}\text { Conductivity } \\
(\mu \mathrm{S} / \mathrm{cm})\end{array}$ & $\begin{array}{c}\text { Dissolved } \\
\text { Oxygen }\end{array}$ & $\begin{array}{l}\text { Carbon } \\
\text { Dioxide }\end{array}$ & Iron(II) & \\
\hline \multirow[t]{6}{*}{ Stone } & $43^{b}$ & $6 / 4 / 04$ & 17.1 & 7.35 & 682 & - & - & - & - \\
\hline & & $9 / 14 / 05$ & 17.3 & 6.81 & 638 & - & - & - & - \\
\hline & & $3 / 19 / 06$ & 12.9 & 6.42 & 650 & - & - & 0 & 213 \\
\hline & & $9 / 19 / 06$ & 16.7 & 7.12 & 639 & - & - & - & - \\
\hline & & $3 / 22 / 07$ & 16.7 & 6.58 & 679 & 4.71 & 35 & 0.28 & 19 \\
\hline & & $10 / 3 / 07$ & 16.1 & 6.97 & 564 & 7.07 & 25 & 0.07 & 225 \\
\hline
\end{tabular}

a Not measured.

b Total depth. 
TABLE 3.4 Results of attenuation parameter analyses for groundwater samples collected at Morrill, October 2003 to October 2007.

\begin{tabular}{|c|c|c|c|c|c|c|c|c|c|c|c|c|c|c|}
\hline \multirow[b]{2}{*}{ Location } & \multirow[b]{2}{*}{$\begin{array}{l}\text { Screen } \\
\text { Interval } \\
\text { (ft BGL) }\end{array}$} & \multirow[b]{2}{*}{$\begin{array}{l}\text { Sample } \\
\text { Date }\end{array}$} & \multicolumn{12}{|c|}{ Concentration $(\mathrm{mg} / \mathrm{L})$} \\
\hline & & & $\begin{array}{c}\text { Total } \\
\text { Alkalinity }\end{array}$ & Chloride & Sulfate & Sulfide & Nitrate & Nitrite & $\begin{array}{l}\text { Nitrate/Nitrite } \\
\text { Nitrogen }\end{array}$ & Phosphate & Aluminum & Calcium & Iron & Magnesium \\
\hline \multirow[t]{6}{*}{ MW1S } & $11-51$ & $10 / 23 / 03$ & 323 & 67.8 & 32.5 & - & 14.3 & - & 13.5 & $\mathrm{U}<0.2^{\mathrm{a}}$ & $U<0.2$ & 123 & $U<0.1$ & 31.4 \\
\hline & & $6 / 2 / 04$ & - & 84.6 & 35.1 & - & 11.2 & $U<0.005$ & 11.8 & $U<0.2$ & $U<0.2$ & 118 & $U<0.1$ & 27.5 \\
\hline & & $9 / 13 / 05$ & 304 & 85.4 & 31.1 & $U<0.02$ & 14.2 & 0.0212 & 14.5 & $U<0.2$ & $U<0.2$ & 126 & $U<0.1$ & 28.2 \\
\hline & & $3 / 22 / 06$ & 311 & 87.0 & 39.3 & $U<0.02$ & 15.2 & $U<0.005$ & 14.2 & $U<0.2$ & $U<0.2$ & 120 & $U<0.1$ & 27.8 \\
\hline & & $3 / 21 / 07$ & 300 & 79.0 & 32.0 & $U<0.02$ & $11.0 \mathrm{H}^{\mathrm{b}}$ & - & 12.0 & $U<0.2$ & $U<0.2$ & 114 & $U<0.1$ & 23.8 \\
\hline & & 10/1/07 & 290 & 170 & 40.0 & $U<0.02$ & 6.60 & - & 14.0 & $U<0.2$ & $U<0.2$ & 116 & $U<0.1$ & 26.3 \\
\hline \multirow[t]{7}{*}{ MW1D } & $63-88$ & $10 / 22 / 03$ & 278 & 19.0 & 1430 & - & $\mathrm{U}<0.2$ & - & $U<0.1$ & $U<0.2$ & $U<0.2$ & 572 & 0.102 & 49.5 \\
\hline & & $6 / 2 / 04$ & - & 18.5 & 1340 & - & $\mathrm{U}<0.2$ & $U<0.005$ & $\mathrm{U}<0.1$ & $U<0.2$ & $U<0.2$ & 582 & $U<0.1$ & 52.0 \\
\hline & & 9/13/05 & - & - & - & - & - & - & - & - & - & - & - & - \\
\hline & & $3 / 19 / 06$ & - & - & - & - & - & - & - & - & - & - & - & - \\
\hline & & 9/20/06 & - & - & - & - & - & - & - & - & - & - & - & - \\
\hline & & $3 / 21 / 07$ & 270 & 23.0 & 1500 & $U<0.02$ & $U<0.1$ & - & $U<0.1$ & $U<0.2$ & $U<0.2$ & 567 & 0.180 & 47.6 \\
\hline & & $10 / 1 / 07$ & 180 & 29.0 & 630 & 0.053 & 2.40 & - & $\mathrm{U}<0.1$ & $\mathrm{U}<0.2$ & $U<0.2$ & 606 & 0.277 & 50.8 \\
\hline \multirow[t]{7}{*}{ MW2S } & $13-53$ & $10 / 22 / 03$ & 295 & 23.2 & 44.0 & - & 25.1 & - & 24.2 & $U<0.2$ & $U<0.2$ & 129 & $U<0.1$ & 31.0 \\
\hline & & $6 / 2 / 04$ & - & 17.1 & 61.8 & - & 16.2 & $U<0.005$ & 17.2 & $U<0.2$ & $U<0.2$ & 124 & $U<0.1$ & 25.8 \\
\hline & & $9 / 14 / 05$ & 289 & 11.0 & 56.6 & $U<0.02$ & 18.2 & $U<0.005$ & 20.4 & 0.307 & $U<0.2$ & 130 & $U<0.1$ & 21.4 \\
\hline & & $3 / 21 / 06$ & 301 & 23.3 & 51.0 & $U<0.02$ & 25.4 & $U<0.005$ & 25.2 & $U<0.2$ & $U<0.2$ & 125 & $U<0.1$ & 31.4 \\
\hline & & 9/18/06 & 306 & 15.1 & 71.3 & $U<0.02$ & 12.4 & - & 14.5 & $U<0.2$ & $U<0.2$ & 124 & $U<0.1$ & 24.3 \\
\hline & & 3/22/07 & 280 & 13.0 & 40.0 & $U<0.02$ & $19.0 \mathrm{H}$ & - & 20.0 & $U<0.2$ & $U<0.2$ & 102 & $U<0.1$ & 23.8 \\
\hline & & 10/3/07 & 250 & 8.20 & 39.0 & $U<0.02$ & 19.0 & - & 22.0 & $U<0.2$ & $U<0.2$ & 112 & $\mathrm{U}<0.1$ & 16.8 \\
\hline \multirow[t]{7}{*}{ MW3S } & $18-48$ & $10 / 23 / 03$ & 298 & 3.38 & 19.7 & - & 12.1 & - & 11.5 & $U<0.2$ & $U<0.2$ & 93.4 & $U<0.1$ & 17.5 \\
\hline & & $6 / 2 / 04$ & - & 3.12 & 20.6 & - & 10.1 & $U<0.005$ & 10.9 & $\mathrm{U}<0.2$ & $\mathrm{U}<0.2$ & 92.6 & $\mathrm{U}<0.1$ & 15.8 \\
\hline & & 9/13/05 & 280 & 3.28 & 28.0 & $U<0.02$ & 13.5 & $U<0.005$ & 14.6 & 0.374 & $U<0.2$ & 106 & $U<0.1$ & 15.6 \\
\hline & & 3/23/06 & 287 & 3.85 & 22.8 & $U<0.02$ & 15.2 & $U<0.005$ & 13.4 & $U<0.2$ & $U<0.2$ & 90.5 & $U<0.1$ & 15.5 \\
\hline & & $9 / 20 / 06$ & 294 & 4.18 & 23.8 & $U<0.02$ & 12.3 & - & 11.5 & $U<0.2$ & $U<0.2$ & 94.7 & $U<0.1$ & 14.3 \\
\hline & & $3 / 22 / 07$ & 290 & 4.40 & 23.0 & $U<0.02$ & $11.0 \mathrm{H}$ & - & 12.0 & $U<0.2$ & $U<0.2$ & 93.4 & $U<0.1$ & 14.4 \\
\hline & & $10 / 3 / 07$ & 280 & 5.30 & 23.0 & $U<0.02$ & 13.0 & - & 14.0 & $U<0.2$ & $U<0.2$ & 100 & $U<0.1$ & 14.7 \\
\hline \multirow[t]{5}{*}{ MW4S } & $17-47$ & $10 / 21 / 03$ & 327 & 11.1 & 21.3 & - & 19.9 & - & 18.3 & $U<0.2$ & $U<0.2$ & 89.5 & $U<0.1$ & 36.0 \\
\hline & & $6 / 4 / 04$ & - & 10.2 & 23.5 & - & 18.9 & $U<0.005$ & 19.3 & $U<0.2$ & $U<0.2$ & 86.2 & $U<0.1$ & 36.6 \\
\hline & & 9/14/05 & 292 & 10.3 & 24.6 & $U<0.02$ & 20.8 & $U<0.005$ & 22.9 & 0.271 & $U<0.2$ & 92.5 & $U<0.1$ & 34.1 \\
\hline & & 3/21/06 & 222 & 9.11 & 18.2 & $U<0.02$ & 16.2 & $U<0.005$ & 15.2 & $U<0.2$ & $U<0.2$ & 55.0 & $U<0.1$ & 23.4 \\
\hline & & 9/18/06 & 298 & 11.0 & 22.2 & $U<0.02$ & 19.6 & - & 18.9 & $U<0.2$ & - & - & - & - \\
\hline
\end{tabular}


TABLE 3.4 (Cont.)

\begin{tabular}{|c|c|c|c|c|c|c|c|c|c|c|c|c|c|c|}
\hline \multirow[b]{2}{*}{ Location } & \multirow{2}{*}{$\begin{array}{l}\text { Screen } \\
\text { Interval } \\
\text { (ft BGL) }\end{array}$} & \multirow[b]{2}{*}{$\begin{array}{l}\text { Sample } \\
\text { Date }\end{array}$} & \multicolumn{12}{|c|}{ Concentration (mg/L) } \\
\hline & & & $\begin{array}{c}\text { Total } \\
\text { Alkalinity }\end{array}$ & Chloride & Sulfate & Sulfide & Nitrate & Nitrite & $\begin{array}{l}\text { Nitrate/Nitrite } \\
\text { Nitrogen }\end{array}$ & Phosphate & Aluminum & Calcium & Iron & Magnesium \\
\hline MW5S & $15-55$ & $\begin{array}{r}10 / 22 / 03 \\
6 / 2 / 04 \\
9 / 13 / 05 \\
3 / 22 / 06 \\
9 / 20 / 06 \\
3 / 22 / 07 \\
10 / 3 / 07\end{array}$ & $\begin{array}{c}308 \\
- \\
292 \\
297 \\
295 \\
310 \\
310\end{array}$ & $\begin{array}{l}12.2 \\
11.7 \\
11.4 \\
13.1 \\
11.1 \\
12.0 \\
22.0\end{array}$ & $\begin{array}{l}53.3 \\
48.7 \\
41.4 \\
48.5 \\
37.0 \\
38.0 \\
65.0\end{array}$ & $\begin{array}{c}- \\
- \\
U<0.02 \\
U<0.02 \\
U<0.02 \\
U<0.02 \\
U<0.02\end{array}$ & $\begin{array}{c}20.4 \\
19.5 \\
18.3 \\
22.0 \\
19.8 \\
20.0 \mathrm{H} \\
14.0\end{array}$ & $\begin{array}{c}-\overline{0} \\
U<0.005 \\
0.0148 \\
U<0.005 \\
\quad- \\
\quad- \\
-\end{array}$ & $\begin{array}{l}19.1 \\
21.0 \\
20.9 \\
20.1 \\
19.2 \\
21.0 \\
15.0\end{array}$ & $\begin{array}{l}U<0.2 \\
U<0.2 \\
U<0.2 \\
U<0.2 \\
U<0.2 \\
U<0.2 \\
U<0.2\end{array}$ & $\begin{array}{l}U<0.2 \\
U<0.2 \\
U<0.2 \\
U<0.2 \\
U<0.2 \\
U<0.2 \\
U<0.2\end{array}$ & $\begin{array}{l}117 \\
106 \\
102 \\
99.1 \\
98.0 \\
95.6 \\
128\end{array}$ & $\begin{array}{l}U<0.1 \\
U<0.1 \\
U<0.1 \\
U<0.1 \\
U<0.1 \\
U<0.1 \\
U<0.1\end{array}$ & $\begin{array}{l}31.6 \\
30.5 \\
29.3 \\
28.4 \\
28.5 \\
27.7 \\
26.0\end{array}$ \\
\hline MW6S & $10-25$ & $\begin{array}{r}6 / 3 / 04 \\
9 / 14 / 05 \\
3 / 20 / 06 \\
9 / 18 / 06 \\
3 / 21 / 07 \\
10 / 2 / 07\end{array}$ & $\begin{array}{l}- \\
261 \\
263 \\
264 \\
270 \\
250\end{array}$ & $\begin{array}{l}25.8 \\
27.2 \\
31.4 \\
26.0 \\
28.0 \\
29.0\end{array}$ & $\begin{array}{l}1280 \\
1330 \\
1560 \\
1330 \\
1400 \\
1300\end{array}$ & $\begin{array}{c}- \\
0.0443 \\
U<0.02 \\
U<0.02 \\
U<0.02 \\
U<0.02\end{array}$ & $\begin{array}{c}0.339 \\
U<0.4 \\
0.321 \\
0.353 \\
U<0.1 \\
U<0.1\end{array}$ & $\begin{array}{c}U<0.005 \\
U<0.005 \\
0.0061 \\
- \\
- \\
-\end{array}$ & $\begin{array}{l}0.0694 \\
U<0.1 \\
U<0.1 \\
0.0142 \\
U<0.1 \\
U<0.1\end{array}$ & $\begin{array}{c}U<0.2 \\
0.272 \\
U<0.2 \\
U<0.2 \\
U<0.2 \\
U<0.2\end{array}$ & $\begin{array}{l}U<0.2 \\
U<0.2 \\
U<0.2 \\
U<0.2 \\
U<0.2 \\
U<0.2\end{array}$ & $\begin{array}{l}470 \\
481 \\
413 \\
461 \\
452 \\
481\end{array}$ & $\begin{array}{l}U<0.1 \\
U<0.1 \\
U<0.1 \\
0.13 B^{c} \\
U<0.1 \\
U<0.1\end{array}$ & $\begin{array}{r}111 \\
104 \\
86.2 \\
98.5 \\
93.8 \\
97.0\end{array}$ \\
\hline MW7S & $20-45$ & $\begin{array}{r}6 / 3 / 04 \\
9 / 12 / 05 \\
3 / 22 / 06 \\
9 / 19 / 06 \\
3 / 20 / 07 \\
10 / 1 / 07\end{array}$ & $\begin{array}{c}- \\
303 \\
304 \\
333 \\
290 \\
240\end{array}$ & $\begin{array}{l}12.8 \\
12.0 \\
9.76 \\
9.73 \\
11.0 \\
11.0\end{array}$ & $\begin{array}{l}19.3 \\
23.3 \\
25.8 \\
21.9 \\
20.0 \\
36.0\end{array}$ & $\begin{array}{c}\quad- \\
U<0.02 \\
U<0.02 \\
U<0.02 \\
U<0.02 \\
U<0.02\end{array}$ & $\begin{array}{c}16.7 \\
18.0 \\
19.8 \\
18.3 \\
17.0 \mathrm{H} \\
17.0\end{array}$ & $\begin{array}{c}0.0089 \\
U<0.005 \\
U<0.005 \\
\quad- \\
- \\
-\end{array}$ & $\begin{array}{l}17.6 \\
19.8 \\
18.4 \\
18.3 \\
17.0 \\
18.0\end{array}$ & $\begin{array}{c}U<0.2 \\
U<0.2 \\
U<0.2 \\
U<0.2 \\
U<0.2 \\
0.270\end{array}$ & $\begin{array}{l}U<0.2 \\
U<0.2 \\
U<0.2 \\
U<0.2 \\
U<0.2 \\
U<0.2\end{array}$ & $\begin{array}{c}100 \\
105 \\
96.9 \\
101 \\
96.3 \\
103\end{array}$ & $\begin{array}{l}U<0.1 \\
U<0.1 \\
U<0.1 \\
U<0.1 \\
U<0.1 \\
U<0.1\end{array}$ & $\begin{array}{l}21.9 \\
22.4 \\
20.4 \\
21.6 \\
20.5 \\
23.4\end{array}$ \\
\hline MW8S & $10-25$ & $\begin{array}{r}6 / 3 / 04 \\
9 / 14 / 05 \\
3 / 20 / 06 \\
9 / 19 / 06 \\
3 / 20 / 07 \\
10 / 2 / 07\end{array}$ & $\begin{array}{c}- \\
285 \\
302 \\
295 \\
300 \\
280\end{array}$ & $\begin{array}{l}14.3 \\
41.6 \\
26.8 \\
37.9 \\
35.0 \\
35.0\end{array}$ & $\begin{array}{l}163 \\
139 \\
159 \\
108 \\
130 \\
130\end{array}$ & $\begin{array}{c}\quad- \\
U<0.02 \\
U<0.02 \\
U<0.02 \\
U<0.02 \\
U<0.02\end{array}$ & $\begin{array}{c}5.85 \\
10.4 \\
8.97 \\
13.8 \\
10.0 \mathrm{H} \\
9.90\end{array}$ & $\begin{array}{c}U<0.005 \\
U<0.005 \\
0.0109 \\
\quad- \\
- \\
-\end{array}$ & $\begin{array}{l}6.52 \\
11.7 \\
8.39 \\
12.9 \\
8.10 \\
11.0\end{array}$ & $\begin{array}{c}U<0.2 \\
0.328 \\
U<0.2 \\
U<0.2 \\
U<0.2 \\
U<0.2\end{array}$ & $\begin{array}{c}U<0.2 \\
- \\
U<0.2 \\
U<0.2 \\
U<0.2 \\
U<0.2\end{array}$ & $\begin{array}{c}130 \\
- \\
110 \\
115 \\
120 \\
129\end{array}$ & $\begin{array}{c}U<0.1 \\
- \\
U<0.1 \\
U<0.1 \\
U<0.1 \\
U<0.1\end{array}$ & $\begin{array}{c}35.2 \\
- \\
28.5 \\
29.0 \\
34.0 \\
34.7\end{array}$ \\
\hline MW9S & $38.83-53.83$ & $\begin{array}{l}3 / 22 / 06 \\
9 / 19 / 06 \\
3 / 20 / 07 \\
10 / 1 / 07\end{array}$ & $\begin{array}{c}350 \\
- \\
290 \\
300\end{array}$ & $\begin{array}{l}5.97 \\
5.86 \\
5.90 \\
6.20\end{array}$ & $\begin{array}{l}57.1 \\
40.9 \\
21.0 \\
12.0\end{array}$ & $\begin{array}{l}U<0.02 \\
U<0.02 \\
U<0.02 \\
U<0.02\end{array}$ & $\begin{array}{c}0.349 \\
1.04 \\
3.90 \mathrm{H} \\
10.0\end{array}$ & $\begin{array}{c}0.0066 \\
- \\
- \\
-\end{array}$ & $\begin{array}{c}0.0427 \\
0.916 \\
8.40 \\
9.50\end{array}$ & $\begin{array}{l}U<0.2 \\
U<0.2 \\
U<0.2 \\
U<0.2\end{array}$ & $\begin{array}{l}U<0.2 \\
U<0.2 \\
U<0.2 \\
U<0.2\end{array}$ & $\begin{array}{l}76.9 \\
79.0 \\
75.5 \\
81.4\end{array}$ & $\begin{array}{l}U<0.1 \\
U<0.1 \\
U<0.1 \\
U<0.1\end{array}$ & $\begin{array}{l}33.7 \\
34.8 \\
31.2 \\
33.8\end{array}$ \\
\hline MW10S & $30-45$ & $\begin{array}{l}3 / 21 / 06 \\
9 / 18 / 06 \\
3 / 21 / 07 \\
10 / 1 / 07\end{array}$ & $\begin{array}{l}351 \\
322 \\
360 \\
330\end{array}$ & $\begin{array}{l}6.07 \\
5.95 \\
5.80 \\
5.70\end{array}$ & $\begin{array}{l}42.0 \\
39.2 \\
30.0 \\
16.0\end{array}$ & $\begin{array}{l}U<0.02 \\
U<0.02 \\
U<0.02 \\
U<0.02\end{array}$ & $\begin{array}{c}0.357 \\
0.411 \\
1.60 \mathrm{H} \\
5.60\end{array}$ & $\begin{array}{c}U<0.005 \\
- \\
- \\
-\end{array}$ & $\begin{array}{c}0.162 \\
U<0.1 \\
1.50 \\
4.00\end{array}$ & $\begin{array}{l}U<0.2 \\
U<0.2 \\
U<0.2 \\
U<0.2\end{array}$ & $\begin{array}{l}U<0.2 \\
U<0.2 \\
U<0.2 \\
U<0.2\end{array}$ & $\begin{array}{l}86.3 \\
82.9 \\
79.4 \\
83.8\end{array}$ & $\begin{array}{l}U<0.1 \\
U<0.1 \\
U<0.1 \\
U<0.1\end{array}$ & $\begin{array}{l}32.6 \\
31.3 \\
30.3 \\
33.8\end{array}$ \\
\hline
\end{tabular}


TABLE 3.4 (Cont.)

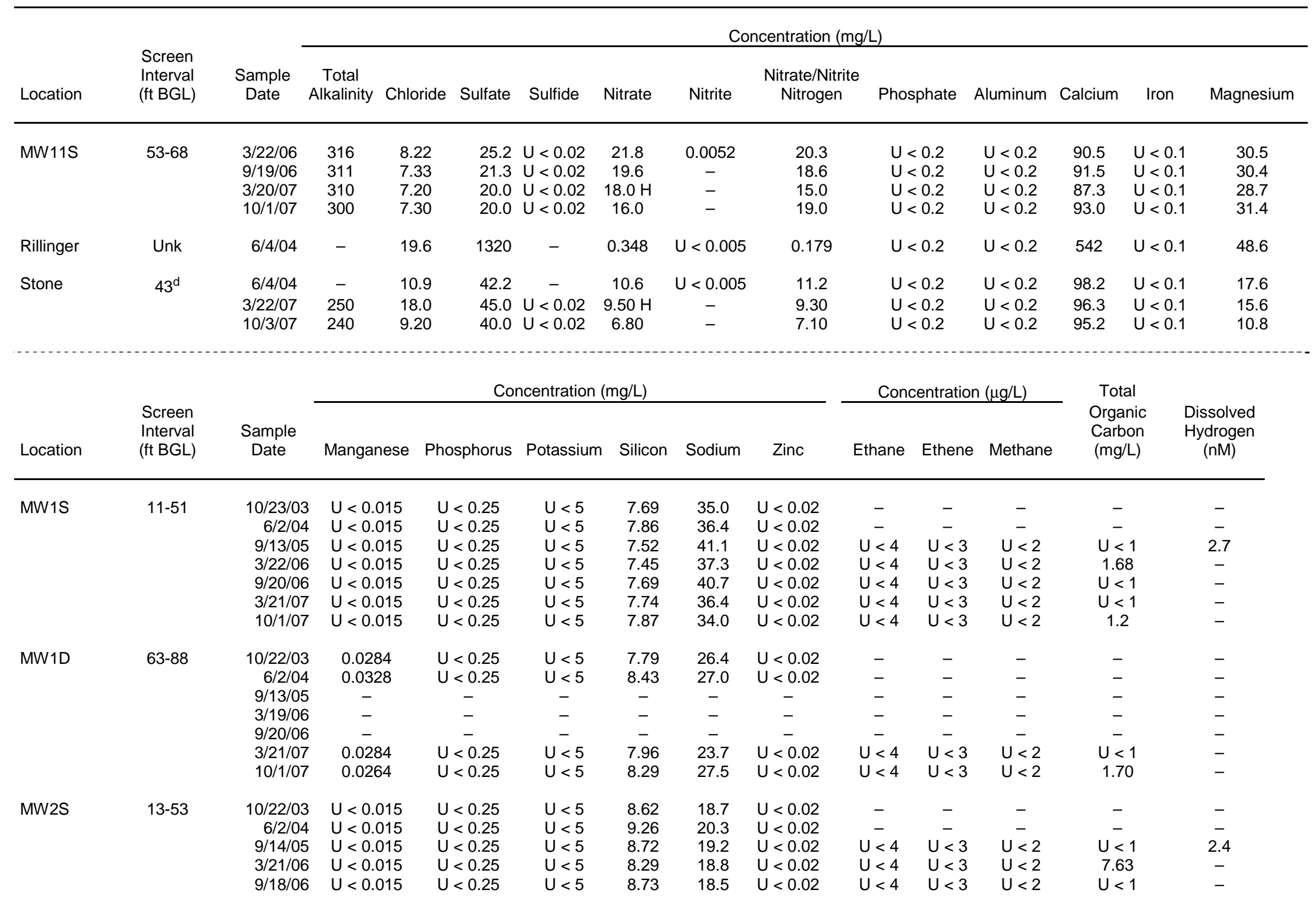


TABLE 3.4 (Cont.)

\begin{tabular}{|c|c|c|c|c|c|c|c|c|c|c|c|c|c|}
\hline \multirow[b]{2}{*}{ Location } & \multirow{2}{*}{$\begin{array}{l}\text { Screen } \\
\text { Interval } \\
\text { (ft BGL) }\end{array}$} & \multirow{2}{*}{$\begin{array}{c}\text { Sample } \\
\text { Date }\end{array}$} & \multicolumn{6}{|c|}{ Concentration $(\mathrm{mg} / \mathrm{L})$} & \multicolumn{3}{|c|}{ Concentration $(\mu \mathrm{g} / \mathrm{L})$} & \multirow{2}{*}{$\begin{array}{c}\text { Total } \\
\text { Organic } \\
\text { Carbon } \\
\text { (mg/L) }\end{array}$} & \multirow{2}{*}{$\begin{array}{l}\text { Dissolvec } \\
\text { Hydrogen } \\
\text { (nM) }\end{array}$} \\
\hline & & & Manganese & Phosphorus & Potassium & Silicon & Sodium & Zinc & Ethane & Ethene & Methane & & \\
\hline MW2S (cont.) & & $\begin{array}{l}3 / 22 / 07 \\
10 / 3 / 07\end{array}$ & $\begin{array}{l}U<0.015 \\
U<0.015\end{array}$ & $\begin{array}{l}U<0.25 \\
U<0.25\end{array}$ & $\begin{array}{l}U<5 \\
U<5\end{array}$ & $\begin{array}{l}8.44 \\
8.74\end{array}$ & $\begin{array}{l}15.9 \\
16.7\end{array}$ & $\begin{array}{l}U<0.02 \\
U<0.02\end{array}$ & $\begin{array}{l}U<4 \\
U<4\end{array}$ & $\begin{array}{l}U<3 \\
U<3\end{array}$ & $\begin{array}{l}U<2 \\
U<2\end{array}$ & $\begin{array}{l}U<1 \\
U<1\end{array}$ & $\begin{array}{l}- \\
-\end{array}$ \\
\hline MW3S & $18-48$ & $\begin{array}{r}10 / 23 / 03 \\
6 / 2 / 04 \\
9 / 13 / 05 \\
3 / 23 / 06 \\
9 / 20 / 06 \\
3 / 22 / 07 \\
10 / 3 / 07\end{array}$ & $\begin{array}{l}U<0.015 \\
U<0.015 \\
U<0.015 \\
U<0.015 \\
U<0.015 \\
U<0.015 \\
U<0.015\end{array}$ & $\begin{array}{l}U<0.25 \\
U<0.25 \\
U<0.25 \\
U<0.25 \\
U<0.25 \\
U<0.25 \\
U<0.25\end{array}$ & $\begin{array}{l}U<5 \\
U<5 \\
U<5 \\
U<5 \\
U<5 \\
U<5 \\
U<5\end{array}$ & $\begin{array}{l}7.34 \\
7.70 \\
7.04 \\
7.27 \\
7.32 \\
7.46 \\
7.76\end{array}$ & $\begin{array}{l}20.2 \\
23.5 \\
25.9 \\
20.1 \\
20.0 \\
20.9 \\
25.0\end{array}$ & $\begin{array}{l}U<0.02 \\
U<0.02 \\
U<0.02 \\
U<0.02 \\
U<0.02 \\
U<0.02 \\
U<0.02\end{array}$ & $\begin{array}{c}- \\
- \\
U<4 \\
U<4 \\
U<4 \\
U<4 \\
U<4\end{array}$ & $\begin{array}{c}- \\
- \\
U<3 \\
U<3 \\
U<3 \\
U<3 \\
U<3\end{array}$ & $\begin{array}{c}- \\
- \\
U<2 \\
U<2 \\
U<2 \\
U<2 \\
U<2\end{array}$ & $\begin{array}{c}- \\
- \\
U<1 \\
1.95 \\
U<1 \\
U<1 \\
U<1\end{array}$ & $\begin{array}{l}- \\
- \\
2.8 \\
- \\
- \\
- \\
-\end{array}$ \\
\hline MW4S & $17-47$ & $\begin{array}{r}10 / 21 / 03 \\
6 / 4 / 04 \\
9 / 14 / 05 \\
3 / 21 / 06 \\
9 / 18 / 06 \\
3 / 22 / 07 \\
10 / 3 / 07\end{array}$ & $\begin{array}{c}U<0.015 \\
U<0.015 \\
U<0.015 \\
U<0.015 \\
U<- \\
U<0.015 \\
U<0.015\end{array}$ & $\begin{array}{c}U<0.25 \\
U<0.25 \\
U<0.25 \\
U<0.25 \\
- \\
U<0.25 \\
U<0.25\end{array}$ & $\begin{array}{c}U<5 \\
U<5 \\
U<5 \\
U<5 \\
- \\
U<5 \\
U<5\end{array}$ & $\begin{array}{c}7.90 \\
8.26 \\
8.01 \\
5.85 \\
- \\
7.88 \\
8.19\end{array}$ & $\begin{array}{c}19.8 \\
18.9 \\
18.0 \\
12.9 \\
- \\
17.5 \\
17.8\end{array}$ & $\begin{array}{c}U<0.02 \\
U<0.02 \\
U<0.02 \\
U<0.02 \\
- \\
U<0.02 \\
U<0.02\end{array}$ & $\begin{array}{c}- \\
- \\
U<4 \\
U<4 \\
U<4 \\
U<4 \\
U<4\end{array}$ & $\begin{array}{c}- \\
- \\
U<3 \\
U<3 \\
U<3 \\
U<3 \\
U<3\end{array}$ & $\begin{array}{c}- \\
- \\
U<2 \\
U<2 \\
U<2 \\
U<2 \\
U<2\end{array}$ & $\begin{array}{c}- \\
- \\
U<1 \\
9.28 \\
U<1 \\
U<1 \\
U<1\end{array}$ & $\begin{array}{l}- \\
- \\
8.5 \\
- \\
- \\
- \\
-\end{array}$ \\
\hline MW5S & $15-55$ & $\begin{array}{r}10 / 22 / 03 \\
6 / 2 / 04 \\
9 / 13 / 05 \\
3 / 22 / 06 \\
9 / 20 / 06 \\
3 / 22 / 07 \\
10 / 3 / 07\end{array}$ & $\begin{array}{l}U<0.015 \\
U<0.015 \\
U<0.015 \\
U<0.015 \\
U<0.015 \\
U<0.015 \\
U<0.015\end{array}$ & $\begin{array}{l}U<0.25 \\
U<0.25 \\
U<0.25 \\
U<0.25 \\
U<0.25 \\
U<0.25 \\
U<0.25\end{array}$ & $\begin{array}{l}U<5 \\
U<5 \\
U<5 \\
U<5 \\
U<5 \\
U<5 \\
U<5\end{array}$ & $\begin{array}{l}8.32 \\
8.32 \\
8.55 \\
7.91 \\
7.79 \\
7.91 \\
8.91\end{array}$ & $\begin{array}{l}19.0 \\
19.0 \\
17.7 \\
16.3 \\
16.3 \\
16.1 \\
20.1\end{array}$ & $\begin{array}{l}U<0.02 \\
U<0.02 \\
U<0.02 \\
U<0.02 \\
U<0.02 \\
U<0.02 \\
U<0.02\end{array}$ & $\begin{array}{c}- \\
- \\
U<4 \\
U<4 \\
U<4 \\
U<4 \\
U<4\end{array}$ & $\begin{array}{c}- \\
- \\
U<3 \\
U<3 \\
U<3 \\
U<3 \\
U<3\end{array}$ & $\begin{array}{c}- \\
- \\
U<2 \\
U<2 \\
U<2 \\
U<2 \\
U<2\end{array}$ & $\begin{array}{c}- \\
- \\
U<1 \\
2.38 \\
U<1 \\
U<1 \\
U<1\end{array}$ & $\begin{array}{l}- \\
- \\
31 \\
- \\
- \\
- \\
-\end{array}$ \\
\hline MW6S & $10-25$ & $\begin{array}{r}6 / 3 / 04 \\
9 / 14 / 05 \\
3 / 20 / 06 \\
9 / 18 / 06 \\
3 / 21 / 07 \\
10 / 2 / 07\end{array}$ & $\begin{array}{c}0.268 \\
U<0.015 \\
0.263 \\
0.301 \\
0.286 \\
0.284\end{array}$ & $\begin{array}{l}U<0.25 \\
U<0.25 \\
U<0.25 \\
U<0.25 \\
U<0.25 \\
U<0.25\end{array}$ & $\begin{array}{l}U<5 \\
U<5 \\
U<5 \\
U<5 \\
U<5 \\
U<5\end{array}$ & $\begin{array}{l}10.4 \\
10.0 \\
9.71 \\
9.75 \\
9.74 \\
10.4\end{array}$ & $\begin{array}{l}36.5 \\
34.2 \\
29.0 \\
31.9 \\
31.0 \\
34.1\end{array}$ & $\begin{array}{c}U<0.02 \\
U<0.02 \\
U<0.02 \\
0.0212 \\
0.0247 \\
U<0.02\end{array}$ & $\begin{array}{c}- \\
U<4 \\
U<4 \\
U<4 \\
U<4 \\
U<4\end{array}$ & $\begin{array}{c}- \\
U<3 \\
U<3 \\
U<3 \\
U<3 \\
U<3\end{array}$ & $\begin{array}{c}- \\
U<2 \\
U<2 \\
U<2 \\
U<2 \\
U<2\end{array}$ & $\begin{array}{c}- \\
U<1 \\
U<1 \\
U<1 \\
U<1 \\
U<1\end{array}$ & $\begin{array}{l}- \\
- \\
- \\
- \\
- \\
-\end{array}$ \\
\hline MW7S & $20-45$ & $\begin{array}{r}6 / 3 / 04 \\
9 / 12 / 05 \\
3 / 22 / 06 \\
9 / 19 / 06 \\
3 / 20 / 07 \\
10 / 1 / 07\end{array}$ & $\begin{array}{l}U<0.015 \\
U<0.015 \\
U<0.015 \\
U<0.015 \\
U<0.015 \\
U<0.015\end{array}$ & $\begin{array}{l}U<0.25 \\
U<0.25 \\
U<0.25 \\
U<0.25 \\
U<0.25 \\
U<0.25\end{array}$ & $\begin{array}{l}U<5 \\
U<5 \\
U<5 \\
U<5 \\
U<5 \\
U<5\end{array}$ & $\begin{array}{l}7.76 \\
7.53 \\
7.26 \\
7.27 \\
7.37 \\
7.66\end{array}$ & \begin{tabular}{l|}
23.9 \\
26.7 \\
20.6 \\
22.0 \\
21.3 \\
23.6
\end{tabular} & $\begin{array}{l}U<0.02 \\
U<0.02 \\
U<0.02 \\
U<0.02 \\
U<0.02 \\
U<0.02\end{array}$ & $\begin{array}{c}- \\
U<4 \\
U<4 \\
U<4 \\
U<4 \\
U<4\end{array}$ & $\begin{array}{c}- \\
U<3 \\
U<3 \\
U<3 \\
U<3 \\
U<3\end{array}$ & $\begin{array}{c}- \\
U<2 \\
U<2 \\
U<2 \\
U<2 \\
U<2\end{array}$ & $\begin{array}{c}- \\
U<1 \\
2.00 \\
U<1 \\
U<1 \\
1.9\end{array}$ & $\begin{array}{l}- \\
7.2 \\
- \\
- \\
- \\
-\end{array}$ \\
\hline
\end{tabular}


TABLE 3.4 (Cont.)

\begin{tabular}{|c|c|c|c|c|c|c|c|c|c|c|c|c|c|}
\hline \multirow[b]{2}{*}{ Location } & \multirow{2}{*}{$\begin{array}{l}\text { Screen } \\
\text { Interval } \\
\text { (ft BGL) }\end{array}$} & \multirow{2}{*}{$\begin{array}{l}\text { Sample } \\
\text { Date }\end{array}$} & \multicolumn{6}{|c|}{ Concentration $(\mathrm{mg} / \mathrm{L})$} & \multicolumn{3}{|c|}{ Concentration $(\mu \mathrm{g} / \mathrm{L})$} & \multirow{2}{*}{$\begin{array}{c}\text { Total } \\
\text { Organic } \\
\text { Carbon } \\
\text { (mg/L) }\end{array}$} & \multirow{2}{*}{$\begin{array}{l}\text { Dissolved } \\
\text { Hydrogen } \\
\text { (nM) }\end{array}$} \\
\hline & & & Manganese & Phosphorus & Potassium & Silicon & Sodium & Zinc & Ethane & Ethene & Methane & & \\
\hline MW8S & $10-25$ & $\begin{array}{r}6 / 3 / 04 \\
9 / 14 / 05 \\
3 / 20 / 06 \\
9 / 19 / 06 \\
3 / 20 / 07 \\
10 / 2 / 07\end{array}$ & $\begin{array}{c}U<0.015 \\
- \\
U<0.015 \\
U<0.015 \\
U<0.015 \\
U<0.015\end{array}$ & $\begin{array}{c}U<0.25 \\
- \\
U<0.25 \\
U<0.25 \\
U<0.25 \\
U<0.25\end{array}$ & $\begin{array}{c}U<5 \\
- \\
U<5 \\
U<5 \\
U<5 \\
U<5\end{array}$ & $\begin{array}{l}8.50 \\
- \\
8.22 \\
8.74 \\
8.06 \\
9.34\end{array}$ & $\begin{array}{l}30.6 \\
- \\
24.3 \\
24.0 \\
27.0 \\
28.2\end{array}$ & $\begin{array}{c}U<0.02 \\
- \\
U<0.02 \\
U<0.02 \\
U<0.02 \\
U<0.02\end{array}$ & $\begin{array}{c}- \\
U<4 \\
U<4 \\
U<4 \\
U<4 \\
U<4\end{array}$ & $\begin{array}{c}- \\
U<3 \\
U<3 \\
U<3 \\
U<3 \\
U<3\end{array}$ & $\begin{array}{c}- \\
U<2 \\
U<2 \\
U<2 \\
U<2 \\
U<2\end{array}$ & $\begin{array}{c}- \\
U<1 \\
1.12 \\
1.44 \\
U<1 \\
U<1\end{array}$ & $\begin{array}{l}- \\
- \\
- \\
- \\
- \\
-\end{array}$ \\
\hline MW9S & $38.83-53.83$ & $\begin{array}{l}3 / 22 / 06 \\
9 / 19 / 06 \\
3 / 20 / 07 \\
10 / 1 / 07\end{array}$ & $\begin{array}{c}0.0701 \\
0.0334 \\
U<0.015 \\
U<0.015\end{array}$ & $\begin{array}{l}U<0.25 \\
U<0.25 \\
U<0.25 \\
U<0.25\end{array}$ & $\begin{array}{l}U<5 \\
U<5 \\
U<5 \\
U<5\end{array}$ & $\begin{array}{l}8.71 \\
8.14 \\
7.33 \\
7.64\end{array}$ & $\begin{array}{l}23.6 \\
23.8 \\
20.8 \\
21.9\end{array}$ & $\begin{array}{l}U<0.02 \\
U<0.02 \\
U<0.02 \\
U<0.02\end{array}$ & $\begin{array}{l}U<4 \\
U<4 \\
U<4 \\
U<4\end{array}$ & $\begin{array}{l}U<3 \\
U<3 \\
U<3 \\
U<3\end{array}$ & $\begin{array}{l}U<2 \\
U<2 \\
U<2 \\
U<2\end{array}$ & $\begin{array}{l}1.60 \\
U<1 \\
U<1 \\
2.30\end{array}$ & $\begin{array}{l}- \\
- \\
- \\
-\end{array}$ \\
\hline MW10S & $30-45$ & $\begin{array}{l}3 / 21 / 06 \\
9 / 18 / 06 \\
3 / 21 / 07 \\
10 / 1 / 07\end{array}$ & $\begin{array}{c}0.0644 \\
0.0366 \\
0.0442 \\
U<0.015\end{array}$ & $\begin{array}{l}U<0.25 \\
U<0.25 \\
U<0.25 \\
U<0.25\end{array}$ & $\begin{array}{l}U<5 \\
U<5 \\
U<5 \\
U<5\end{array}$ & $\begin{array}{l}8.90 \\
8.82 \\
7.81 \\
7.92\end{array}$ & $\begin{array}{l}28.2 \\
25.8 \\
23.2 \\
23.2\end{array}$ & $\begin{array}{l}U<0.02 \\
U<0.02 \\
U<0.02 \\
U<0.02\end{array}$ & $\begin{array}{l}U<4 \\
U<4 \\
U<4 \\
U<4\end{array}$ & $\begin{array}{l}U<3 \\
U<3 \\
U<3 \\
U<3\end{array}$ & $\begin{array}{l}U<2 \\
U<2 \\
U<2 \\
U<2\end{array}$ & $\begin{array}{c}4.26 \\
U<1 \\
U<1 \\
1.20\end{array}$ & $\begin{array}{l}- \\
- \\
- \\
-\end{array}$ \\
\hline MW11S & $53-68$ & $\begin{array}{l}3 / 22 / 06 \\
9 / 19 / 06 \\
3 / 20 / 07 \\
10 / 1 / 07\end{array}$ & $\begin{array}{l}U<0.015 \\
U<0.015 \\
U<0.015 \\
U<0.015\end{array}$ & $\begin{array}{l}U<0.25 \\
U<0.25 \\
U<0.25 \\
U<0.25\end{array}$ & $\begin{array}{l}U<5 \\
U<5 \\
U<5 \\
U<5\end{array}$ & $\begin{array}{l}7.48 \\
7.28 \\
7.38 \\
7.86\end{array}$ & $\begin{array}{l}22.6 \\
24.3 \\
23.4 \\
26.9\end{array}$ & $\begin{array}{l}U<0.02 \\
U<0.02 \\
U<0.02 \\
U<0.02\end{array}$ & $\begin{array}{l}U<4 \\
U<4 \\
U<4 \\
U<4\end{array}$ & $\begin{array}{l}U<3 \\
U<3 \\
U<3 \\
U<3\end{array}$ & $\begin{array}{l}U<2 \\
U<2 \\
U<2 \\
U<2\end{array}$ & $\begin{array}{l}2.19 \\
U<1 \\
U<1 \\
2.20\end{array}$ & $\begin{array}{l}- \\
- \\
- \\
-\end{array}$ \\
\hline Rillinger & Unk & $6 / 4 / 04$ & 0.0207 & $U<0.25$ & $U<5$ & 8.36 & 26.6 & $U<0.02$ & - & - & - & - & - \\
\hline Stone & $43^{d}$ & $\begin{array}{r}6 / 4 / 04 \\
3 / 22 / 07 \\
10 / 3 / 07\end{array}$ & $\begin{array}{l}U<0.015 \\
U<0.015 \\
U<0.015\end{array}$ & $\begin{array}{l}U<0.25 \\
U<0.25 \\
U<0.25\end{array}$ & $\begin{array}{l}U<5 \\
U<5 \\
U<5\end{array}$ & $\begin{array}{l}7.87 \\
7.44 \\
7.13\end{array}$ & $\begin{array}{l}15.6 \\
15.6 \\
19.6\end{array}$ & $\begin{array}{l}0.0296 \\
0.0322 \\
0.0993\end{array}$ & $\begin{array}{l}U<4 \\
U<4\end{array}$ & $\begin{array}{l}U<3 \\
U<3\end{array}$ & $\begin{array}{l}U<2 \\
U<2\end{array}$ & $\begin{array}{l}U<1 \\
1.10\end{array}$ & $\begin{array}{l}- \\
- \\
-\end{array}$ \\
\hline
\end{tabular}

a Qualifier U indicates the constituent was not detected at the indicated limit.

b Qualifier $\mathrm{H}$ indicates that the holding time before the analysis was exceeded.

c Qualifier B indicates that the constituent was present in the blank.

d Total depth. 
TABLE 3.5 Results of analyses at the AGEM Laboratory for volatile organic compounds in surface water and sediment samples collected at Morrill in March 2007 to October 2007. ${ }^{a}$

\begin{tabular}{|c|c|c|c|c|c|c|c|}
\hline \multirow[b]{2}{*}{ Location } & \multirow[b]{2}{*}{ Sample } & \multirow[b]{2}{*}{$\begin{array}{l}\text { Sample } \\
\text { Date }\end{array}$} & \multirow[b]{2}{*}{ Medium } & \multicolumn{4}{|c|}{ Concentration ( $\mu \mathrm{g} / \mathrm{L}$ in water; $\mu \mathrm{g} / \mathrm{kg}$ in sediment) } \\
\hline & & & & $\begin{array}{c}\text { Carbon } \\
\text { Tetrachloride }\end{array}$ & Chloroform & $\begin{array}{c}\text { Methylene } \\
\text { Chloride }\end{array}$ & $\begin{array}{c}\text { Quantitation } \\
\text { Limit }\end{array}$ \\
\hline SM1 & MRSM1-W-16572 & $3 / 22 / 07$ & Water & $N D^{b}$ & ND & ND & 1.0 \\
\hline SM1 & MRSM1-S-16573 & $3 / 22 / 07$ & Sediment & ND & ND & ND & 10 \\
\hline SM1 & MRSM1-W-16583 & $10 / 8 / 07$ & Water & ND & ND & ND & 1.0 \\
\hline SM1 & MRSM1-S-16584 & $10 / 8 / 07$ & Sediment & ND & ND & ND & 10 \\
\hline SM2 & MRSM2-W-16574 & $3 / 22 / 07$ & Water & ND & ND & ND & 1.0 \\
\hline SM2 & MRSM2-S-16575 & $3 / 22 / 07$ & Sediment & ND & ND & ND & 10 \\
\hline SM2 & MRSM2-W-16585 & $10 / 8 / 07$ & Water & ND & ND & ND & 1.0 \\
\hline SM2 & MRSM2-S-16586 & $10 / 8 / 07$ & Sediment & ND & ND & ND & 10 \\
\hline SM3 & MRSM3-W-16576 & $3 / 22 / 07$ & Water & ND & ND & ND & 1.0 \\
\hline SM3 & MRSM3-S-16577 & $3 / 22 / 07$ & Sediment & ND & ND & ND & 10 \\
\hline SM3 & MRSM3-W-16587 & $10 / 8 / 07$ & Water & ND & ND & ND & 1.0 \\
\hline SM3 & MRSM3-S-16588 & $10 / 8 / 07$ & Sediment & ND & ND & ND & 10 \\
\hline SM4 & MRSM4-W-16578 & $3 / 22 / 07$ & Water & ND & ND & ND & 1.0 \\
\hline SM4 & MRSM4-S-16579 & $3 / 22 / 07$ & Sediment & ND & ND & ND & 10 \\
\hline SM4 & MRSM4-W-16589 & $10 / 8 / 07$ & Water & ND & ND & ND & 1.0 \\
\hline SM4 & MRSM4-S-16590 & $10 / 8 / 07$ & Sediment & ND & ND & ND & 10 \\
\hline SMB & MRSMB-W-16570 & $3 / 22 / 07$ & Water & ND & ND & ND & 1.0 \\
\hline SMB & MRSMB-S-16571 & $3 / 22 / 07$ & Sediment & ND & ND & ND & 10 \\
\hline SMB & MRSMB-W-16581 & $10 / 8 / 07$ & Water & ND & ND & ND & 1.0 \\
\hline SMB & MRSMB-S-16582 & $10 / 8 / 07$ & Sediment & ND & ND & ND & 10 \\
\hline
\end{tabular}

a Analyses conducted at the AGEM Laboratory, Argonne, Illinois, with EPA Method 524.2 for surface water samples or modified EPA Method 5030B/8260B for sediment samples.

b ND, not detected at the instrument detection limit of $0.1 \mu \mathrm{g} / \mathrm{L}$ for surface water samples or $1.0 \mu \mathrm{g} / \mathrm{kg}$ for sediment samples. 
TABLE 3.6 Results of analyses at the AGEM Laboratory for volatile organic compounds in vegetation samples collected at Morrill in October 2006 to July $2007 .^{a}$

\begin{tabular}{|c|c|c|c|c|c|}
\hline \multirow[b]{2}{*}{ Location } & \multirow[b]{2}{*}{ Sample } & \multirow[b]{2}{*}{$\begin{array}{l}\text { Sample } \\
\text { Date }\end{array}$} & \multirow[b]{2}{*}{ Type } & \multicolumn{2}{|c|}{ Concentration $(\mu \mathrm{g} / \mathrm{kg})$} \\
\hline & & & & $\begin{array}{c}\text { Carbon } \\
\text { Tetrachloride }\end{array}$ & Chloroform \\
\hline MR001 & MR001-B-18959 & $10 / 14 / 06$ & Branch & $N D^{b}$ & 1.6 \\
\hline MR001 & MR001-L-18958 & $10 / 14 / 06$ & Leaf & ND & 3.3 \\
\hline MR001 & MR001-B-23173 & $4 / 2 / 07$ & Branch & ND & ND \\
\hline MR001 & MR001-B-23213 & $7 / 26 / 07$ & Branch & ND & ND \\
\hline MR001 & MR001-L-23212 & $7 / 26 / 07$ & Leaf & ND & ND \\
\hline MR002 & MR002-B-18961 & $10 / 14 / 06$ & Branch & ND & ND \\
\hline MR002 & MR002-L-18960 & $10 / 14 / 06$ & Leaf & ND & ND \\
\hline MR002 & MR002-B-23174 & $4 / 2 / 07$ & Branch & ND & 1.5 \\
\hline MR002 & MR002-B-23211 & $7 / 26 / 07$ & Branch & ND & ND \\
\hline MR002 & MR002-L-23210 & $7 / 26 / 07$ & Leaf & ND & 1.3 \\
\hline MR003 & MR003-B-18963 & $10 / 14 / 06$ & Branch & ND & 1.5 \\
\hline MR003 & MR003-L-18962 & $10 / 14 / 06$ & Leaf & ND & 1.8 \\
\hline MR003 & MR003-B-23175 & $4 / 2 / 07$ & Branch & ND & ND \\
\hline MR003 & MR003-B-23209 & $7 / 26 / 07$ & Branch & ND & ND \\
\hline MR003 & MR003-L-23208 & $7 / 26 / 07$ & Leaf & ND & 0.8 \\
\hline MR004 & MR004-B-18965 & $10 / 14 / 06$ & Branch & ND & 2.1 \\
\hline MR004 & MR004-L-18964 & $10 / 14 / 06$ & Leaf & ND & ND \\
\hline MR004 & MR004-B-23176 & $4 / 2 / 07$ & Branch & ND & ND \\
\hline MR004 & MR004-B-23205 & $7 / 26 / 07$ & Branch & ND & ND \\
\hline MR004 & MR004-L-23204 & $7 / 26 / 07$ & Leaf & ND & ND \\
\hline MR005 & MR005-B-18967 & $10 / 14 / 06$ & Branch & ND & ND \\
\hline MR005 & MR005-L-18966 & $10 / 14 / 06$ & Leaf & ND & 1.2 \\
\hline MR005 & MR005-B-23177 & $4 / 2 / 07$ & Branch & ND & ND \\
\hline MR005 & MR005-B-23207 & $7 / 26 / 07$ & Branch & ND & ND \\
\hline MR005 & MR005-L-23206 & $7 / 26 / 07$ & Leaf & ND & 1.4 \\
\hline MR006 & MR006-B-18969 & $10 / 14 / 06$ & Branch & ND & 3.8 \\
\hline MR006 & MR006-L-18968 & $10 / 14 / 06$ & Leaf & ND & ND \\
\hline MR006 & MR006-B-23161 & $4 / 2 / 07$ & Branch & ND & ND \\
\hline MR006 & MR006-B-23181 & $7 / 26 / 07$ & Branch & ND & ND \\
\hline MR006 & MR006-L-23180 & $7 / 26 / 07$ & Leaf & ND & 1.3 \\
\hline MR007 & MR007-B-18971 & $10 / 14 / 06$ & Branch & ND & 1.6 \\
\hline MR007 & MR007-L-18970 & $10 / 14 / 06$ & Leaf & ND & 2.0 \\
\hline MR007 & MR007-B-23162 & $4 / 2 / 07$ & Branch & ND & ND \\
\hline MR007 & MR007-B-23183 & $7 / 26 / 07$ & Branch & $<1(0.1)$ & ND \\
\hline MR007 & MR007-L-23182 & $7 / 26 / 07$ & Leaf & ND & 1.3 \\
\hline MR008 & MR008-B-18973 & $10 / 14 / 06$ & Branch & ND & 1.3 \\
\hline MR008 & MR008-L-18972 & $10 / 14 / 06$ & Leaf & ND & 2.5 \\
\hline MR008 & MR008-B-23163 & $4 / 2 / 07$ & Branch & ND & 0.8 \\
\hline MR008 & MR008-B-23185 & $7 / 26 / 07$ & Branch & ND & ND \\
\hline MR008 & MR008-L-23184 & $7 / 26 / 07$ & Leaf & ND & ND \\
\hline
\end{tabular}


TABLE 3.6 (Cont.)

\begin{tabular}{|c|c|c|c|c|c|}
\hline \multirow[b]{2}{*}{ Location } & \multirow[b]{2}{*}{ Sample } & \multirow[b]{2}{*}{$\begin{array}{c}\text { Sample } \\
\text { Date }\end{array}$} & \multirow[b]{2}{*}{ Type } & \multicolumn{2}{|c|}{ Concentration $(\mu \mathrm{g} / \mathrm{kg})$} \\
\hline & & & & $\begin{array}{c}\text { Carbon } \\
\text { Tetrachloride }\end{array}$ & Chloroform \\
\hline MR009 & MR009-B-18975 & $10 / 14 / 06$ & Branch & ND & 0.8 \\
\hline MR009 & MR009-L-18974 & $10 / 14 / 06$ & Leaf & ND & 2.5 \\
\hline MR009 & MR009-B-23165 & $4 / 2 / 07$ & Branch & ND & ND \\
\hline MR009 & MR009-B-23189 & $7 / 26 / 07$ & Branch & ND & ND \\
\hline MR009 & MR009-L-23188 & $7 / 26 / 07$ & Leaf & ND & 1.5 \\
\hline MR010 & MR010-B-18977 & $10 / 14 / 06$ & Branch & ND & 3.4 \\
\hline MR010 & MR010-L-18976 & $10 / 14 / 06$ & Leaf & ND & ND \\
\hline MR010 & MR010-B-23167 & $4 / 2 / 07$ & Branch & ND & ND \\
\hline MR010 & MR010-B-23193 & $7 / 26 / 07$ & Branch & ND & 0.8 \\
\hline MR010 & MR010-L-23192 & $7 / 26 / 07$ & Leaf & ND & 1.4 \\
\hline MR011 & MR011-B-18979 & $10 / 14 / 06$ & Branch & ND & 2.2 \\
\hline MR011 & MR011-L-18978 & $10 / 14 / 06$ & Leaf & ND & 2.6 \\
\hline MR011 & MR011-B-23168 & $4 / 2 / 07$ & Branch & ND & ND \\
\hline MR011 & MR011-B-23195 & $7 / 26 / 07$ & Branch & ND & ND \\
\hline MR011 & MR011-L-23194 & $7 / 26 / 07$ & Leaf & ND & 1.7 \\
\hline MR012 & MR012-B-18981 & $10 / 14 / 06$ & Branch & ND & 2.1 \\
\hline MR012 & MR012-L-18980 & $10 / 14 / 06$ & Leaf & ND & 2.9 \\
\hline MR012 & MR012-B-23169 & $4 / 2 / 07$ & Branch & ND & ND \\
\hline MR012 & MR012-B-23197 & $7 / 26 / 07$ & Branch & ND & ND \\
\hline MR012 & MR012-L-23196 & $7 / 26 / 07$ & Leaf & ND & 1.4 \\
\hline MR013 & MR013-B-18983 & $10 / 14 / 06$ & Branch & ND & ND \\
\hline MR013 & MR013-L-18982 & $10 / 14 / 06$ & Leaf & ND & 2.2 \\
\hline MR013 & MR013-B-23160 & $4 / 2 / 07$ & Branch & ND & ND \\
\hline MR013 & MR013-B-23179 & $7 / 26 / 07$ & Branch & ND & ND \\
\hline MR013 & MR013-L-23178 & $7 / 26 / 07$ & Leaf & ND & ND \\
\hline MR014 & MR014-B-23164 & 4/2/07 & Branch & ND & ND \\
\hline MR014 & MR014-B-23187 & $7 / 26 / 07$ & Branch & $<1(0.28)$ & ND \\
\hline MR014 & MR014-L-23186 & $7 / 26 / 07$ & Leaf & ND & ND \\
\hline MR015 & MR015-B-23166 & 4/2/07 & Branch & ND & 0.8 \\
\hline MR015 & MR015-B-23191 & $7 / 26 / 07$ & Branch & ND & ND \\
\hline MR015 & MR015-L-23190 & $7 / 26 / 07$ & Leaf & ND & ND \\
\hline MR016 & MR016-B-23170 & 4/2/07 & Branch & ND & 1.1 \\
\hline MR016 & MR016-B-23199 & $7 / 26 / 07$ & Branch & ND & ND \\
\hline MR016 & MR016-L-23198 & $7 / 26 / 07$ & Leaf & ND & ND \\
\hline MR017 & MR017-B-23171 & 4/2/07 & Branch & ND & ND \\
\hline MR017 & MR017-B-23203 & $7 / 26 / 07$ & Branch & ND & ND \\
\hline MR017 & MR017-L-23202 & $7 / 26 / 07$ & Leaf & ND & ND \\
\hline MR018 & MR018-B-23172 & $4 / 2 / 07$ & Branch & ND & ND \\
\hline MR018 & MR018-B-23201 & $7 / 26 / 07$ & Branch & ND & ND \\
\hline MR018 & MR018-L-23200 & $7 / 26 / 07$ & Leaf & ND & 3.2 \\
\hline
\end{tabular}


TABLE 3.6 (Cont.)

a Analyses conducted at the AGEM Laboratory, Argonne, Illinois, by using modified EPA Method 5021 (headspace analysis on a gas chromatograph with electron capture detection).

b ND, not detected at the method reporting limit of $0.1 \mu \mathrm{g} / \mathrm{kg}$ for carbon tetrachloride or $0.75 \mu \mathrm{g} / \mathrm{kg}$ for chloroform. 


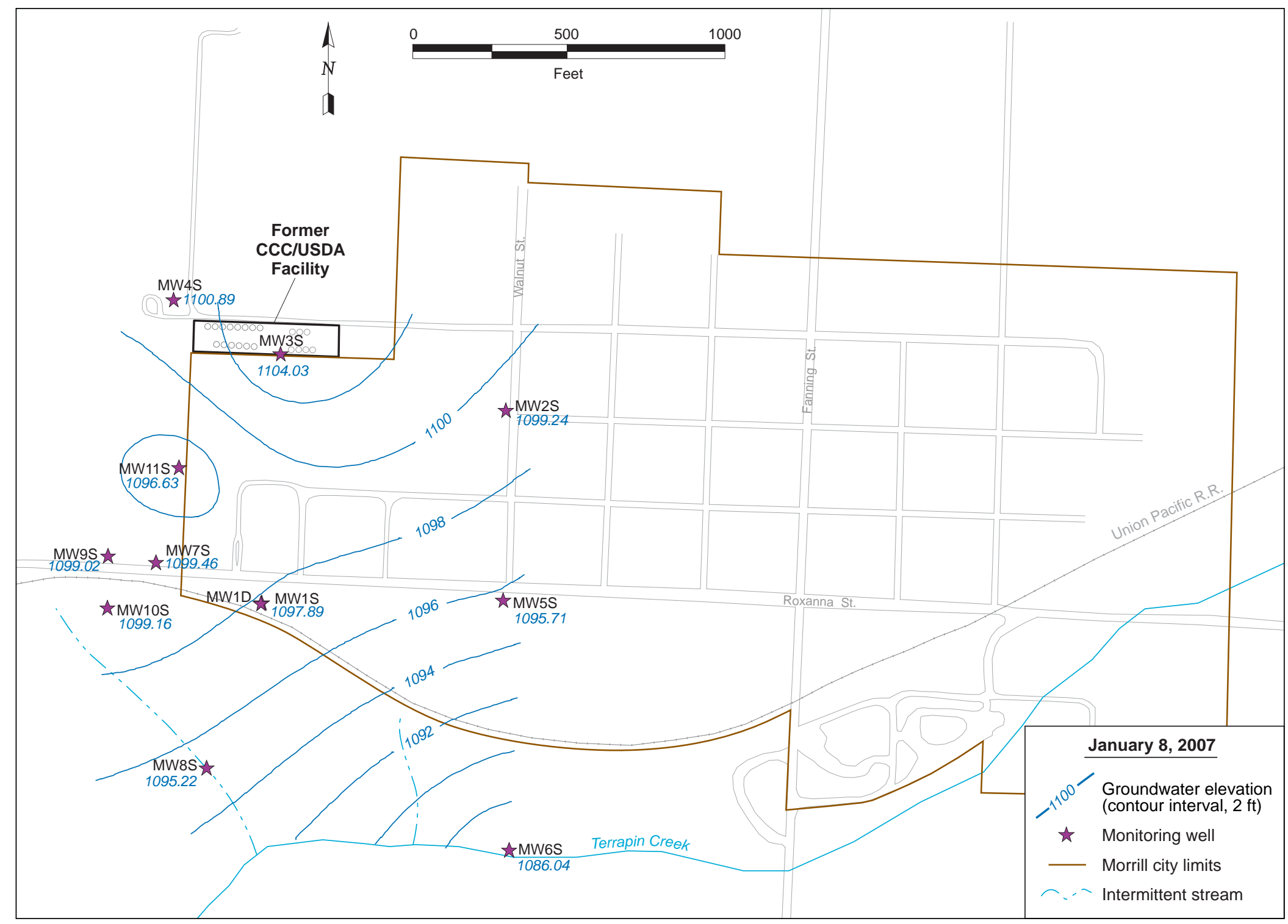

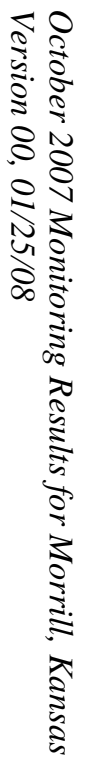

FIGURE 3.1a Potentiometric surface at Morrill, based on water levels measured manually on January 8, 2007. 


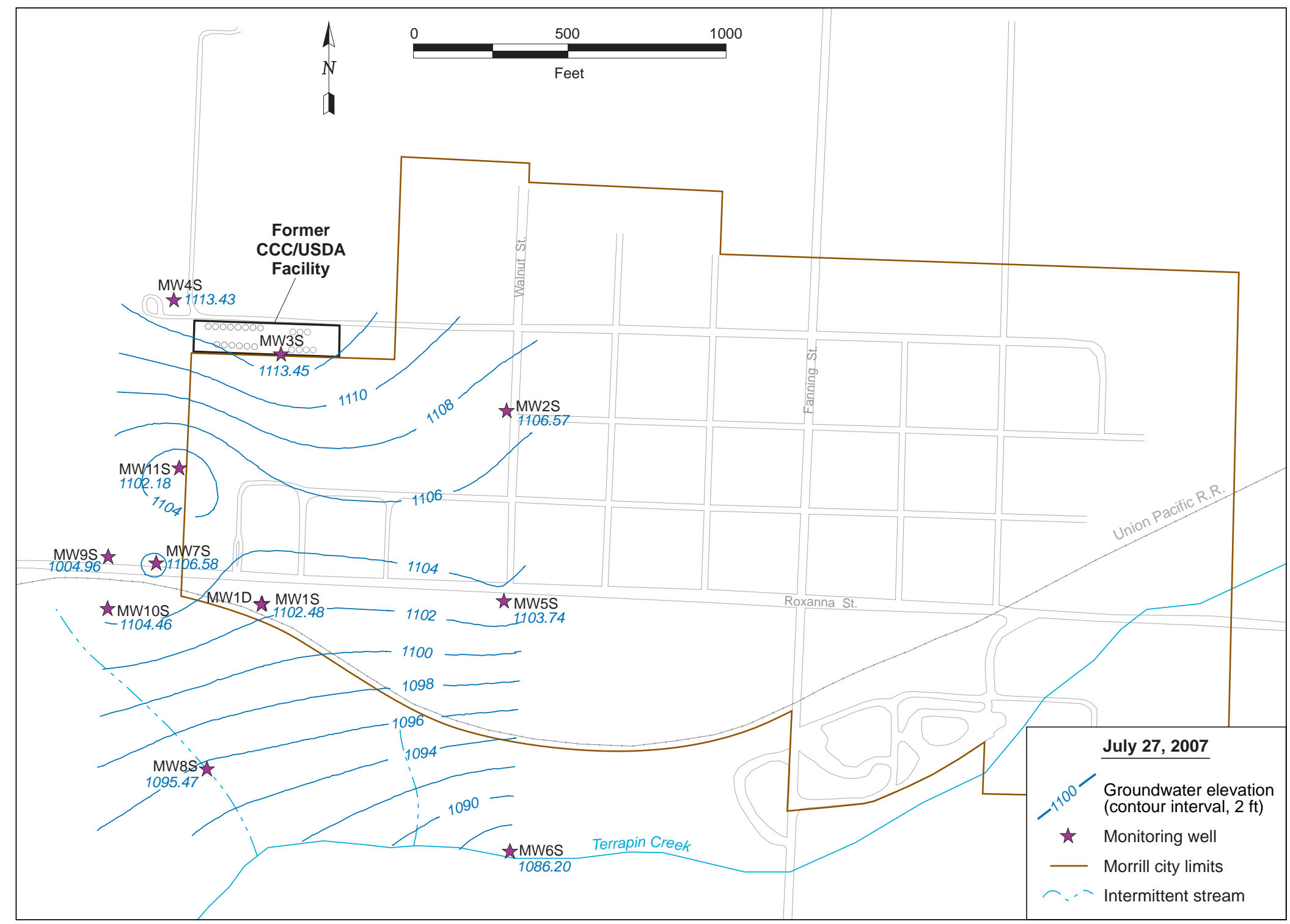

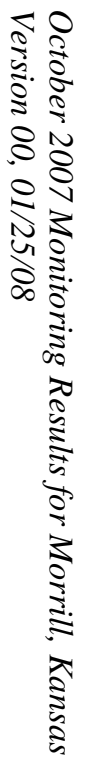

FIGURE 3.1b Potentiometric surface at Morrill, based on water levels measured manually on July 27, 2007. 


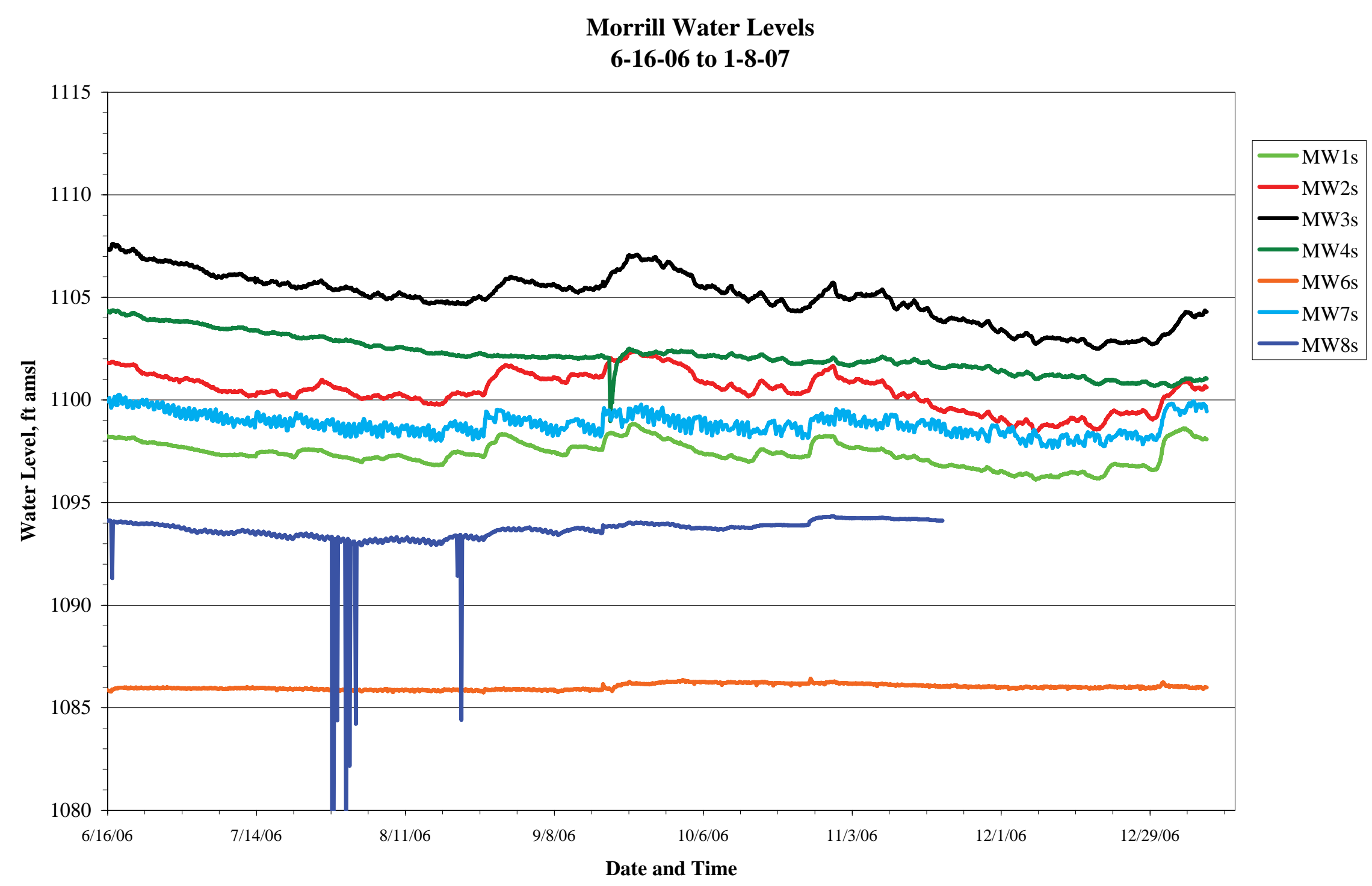

FIGURE 3.2a Hydrographs summarizing results of long-term water level monitoring in wells MW1S-MW4S and MW6S-MW8S at Morrill, from June 16, 2006 to January 8, 2007. 


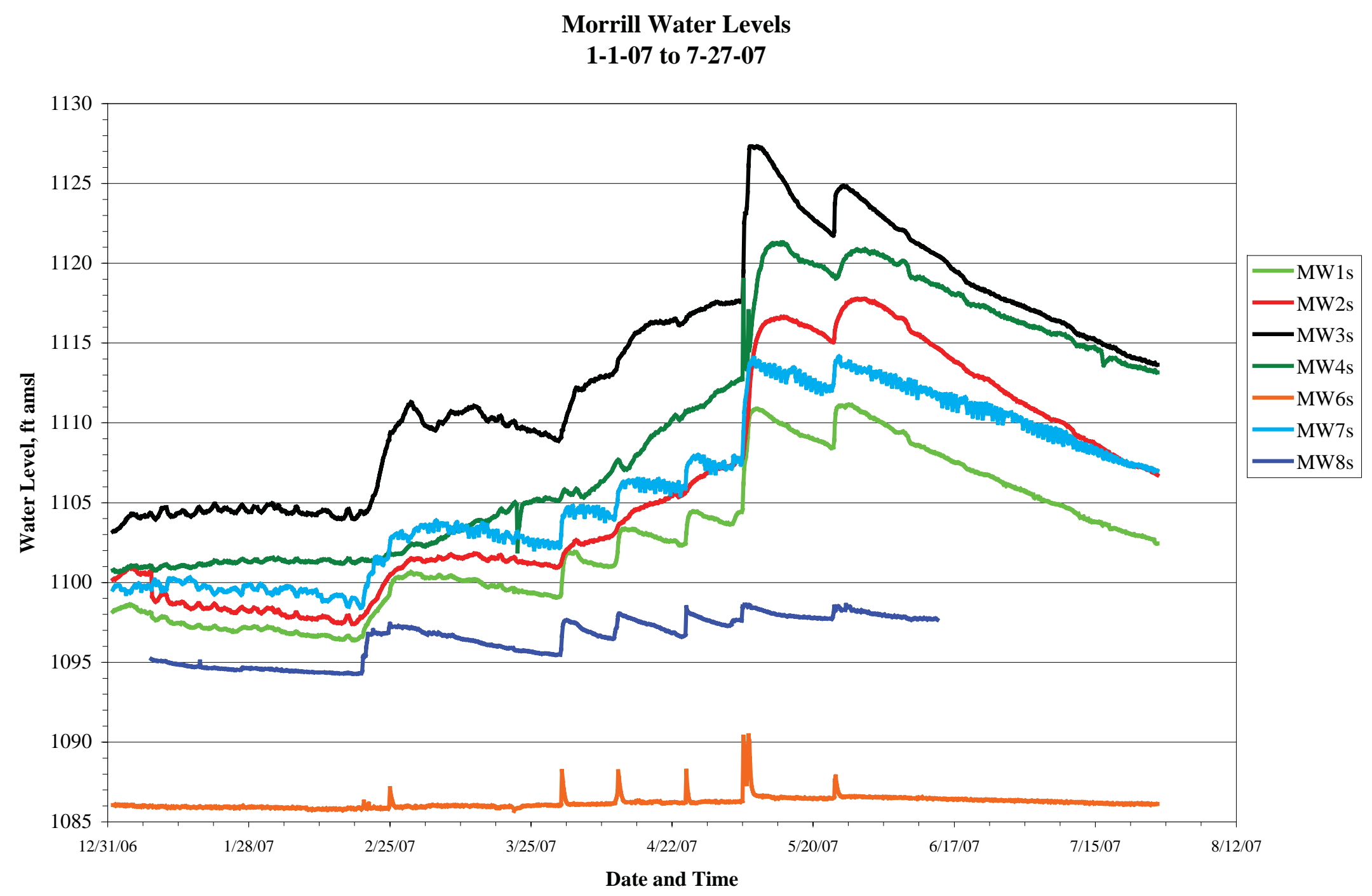

FIGURE 3.2b Hydrographs summarizing results of long-term water level monitoring in wells MW1S-MW4S and MW6S-MW8S at Morrill, from January 1, 2007, to July 27, 2007. 


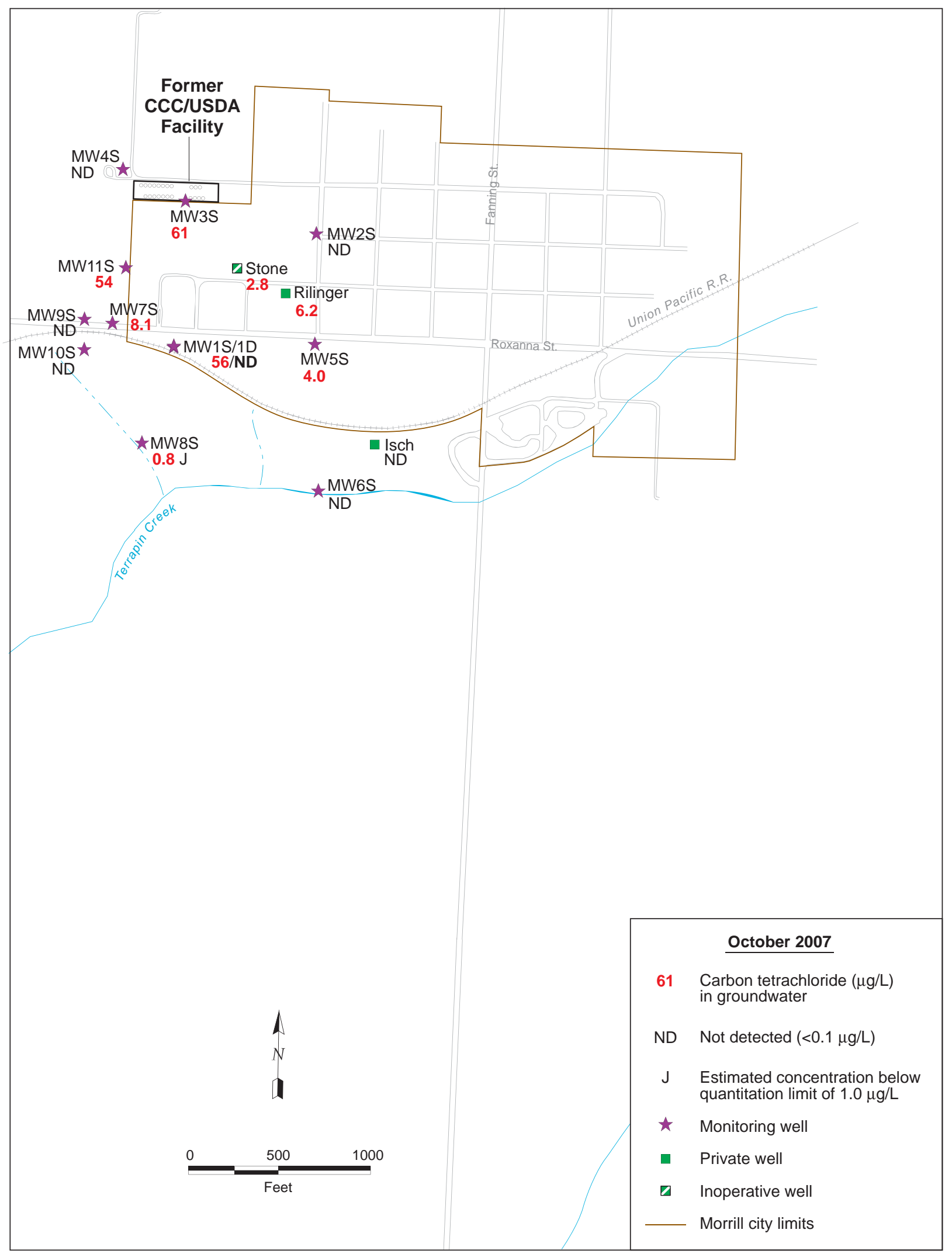

FIGURE 3.3 Carbon tetrachloride levels in groundwater at Morrill, October 2007. 


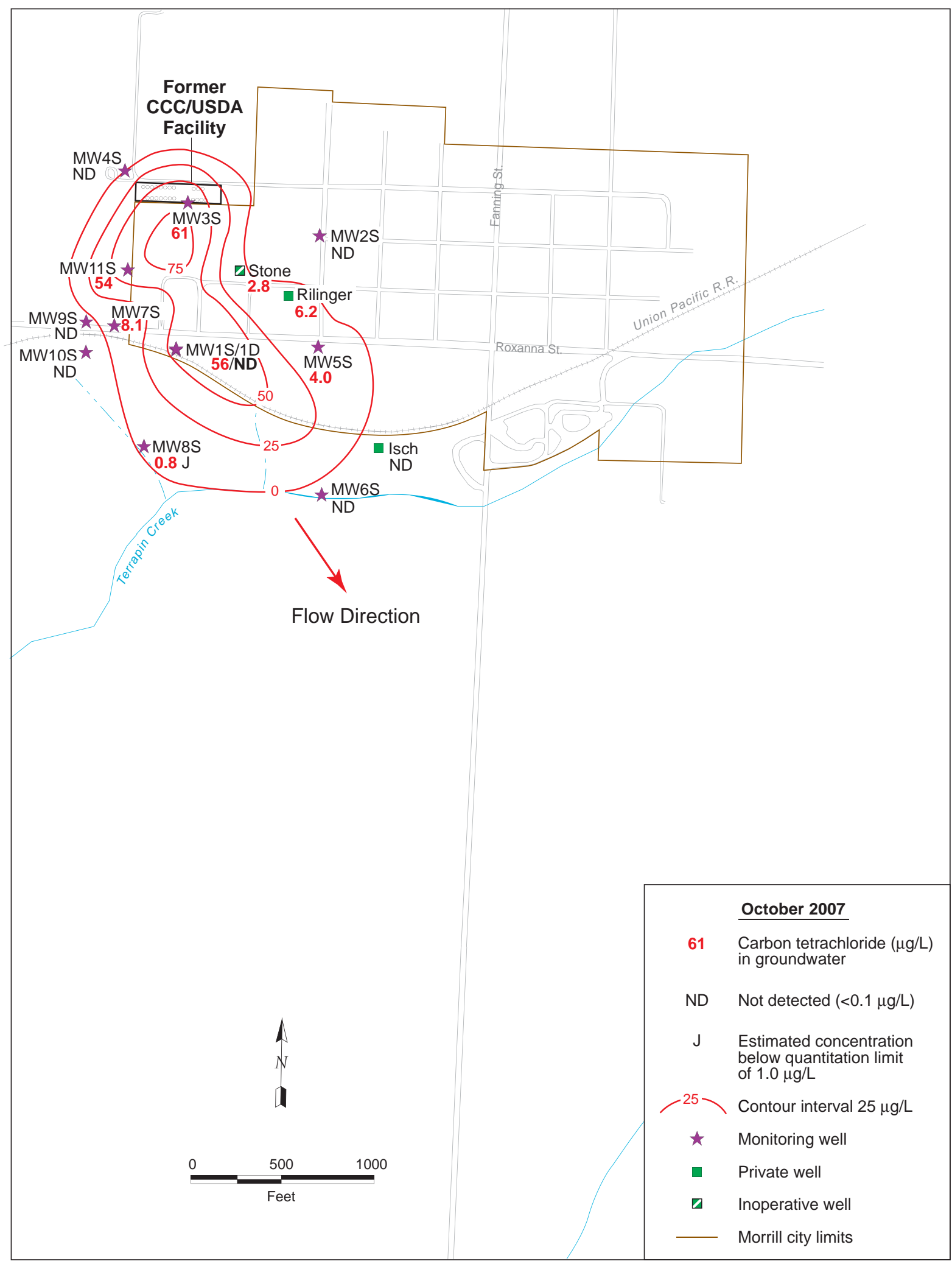

FIGURE 3.4 Lateral extent of the carbon tetrachloride contamination in groundwater at Morrill, as interpreted on the basis of sampling and analysis in October 2007 and the flow direction determined on January 8, 2007, and July 27, 2007. 


\section{Conclusions and Recommendations}

\subsection{Conclusions}

The findings of the October 2007 monitoring event at Morrill support the following conclusions:

- Measurements of groundwater levels made both manually and through the use of automatic recorders have consistently indicated an apparent direction of groundwater flow to the south-southeast from the former CCC/USDA facility. An apparent localized groundwater low near MW11S, southwest of the former facility, has persisted. Sporadic apparent drawdown events observed at MW8S in summer 2006 have not yet recurred.

- The October 2007 well sampling data are generally consistent with previous results. Increases in contaminant concentrations have occurred in monitoring wells MW1S and MW11S and in the Rilinger well since the March 2007 sampling event. Concentrations elsewhere have decreased or remained unchanged. The changes do not define a clear pattern. Since the CCC/USDA began its investigations at this site in 2003, little net change has occurred in the concentrations or distribution of carbon tetrachloride identified in the groundwater at Morrill. Overall, contaminant concentrations have declined considerably from the maximum of $390 \mu \mathrm{g} / \mathrm{L}$ at MW3S detected in 1995, when KDHE monitoring began (GeoCore 1996). Figure 4.1 compares the carbon tetrachloride concentrations in groundwater at Morrill in October 2003 and October 2007.

- Trace levels of carbon tetrachloride have consistently been detected in the shallow groundwater at MW8S, suggesting that the downgradient edge of the plume may lie near this location.

- Sampling to date has shown no substantial evidence for the presence of subsurface conditions at Morrill conducive to anaerobic degradation of the observed carbon tetrachloride contamination in groundwater. 
- No carbon tetrachloride contamination was detected in surface waters or shallow stream bed sediments sampled (at the request of the KDHE) at five locations along Terrapin Creek, downgradient of the former CCC/USDA facility.

- Trace concentrations of carbon tetrachloride were detected in samples of branch tissues collected from mature trees at 2 of 18 locations along Terrapin Creek and its tributaries, south and downgradient of the former CCC/USDA facility. The trace detections in vegetation occurred near MW8S, where the contaminant has occurred consistently in groundwater at trace to low levels.

\subsection{Recommendations}

The October 2007 sampling event is the final event approved by the KDHE under the existing monitoring plan (Argonne 2005b). The following recommendations are based on the results reported here and on previous work at Morrill:

- Semiannual sampling will continue, as requested by the KDHE (2007b).

- Sampling events should continue to include groundwater sampling from the existing network of 12 monitoring wells and 3 private wells, as well as sampling of surface waters and stream bed sediments at 5 established locations along Terrapin Creek.

- Sampling of tree branch tissues from existing mature trees at 18 locations along Terrapin Creek should continue. The distribution of sampling locations and the sampling frequency will be reevaluated as needed on the basis of future sampling results.

- As a consequence of the lack of strong analytical verification for anaerobic degradation (natural attenuation) of the observed carbon tetrachloride in groundwater, future sampling events should have a narrower focus. Analyses for iron(II), sulfate, sulfide, methane, total organic carbon, carbon dioxide, alkalinity, chloride, and dissolved hydrogen should be discontinued. Samples 
should be analyzed only for carbon tetrachloride, chloroform, methylene chloride, dissolved oxygen, nitrate, oxidation-reduction potential, $\mathrm{pH}$, and temperature. Wastewater samples will continue to be analyzed for VOCs, $\mathrm{EDB}$, and nitrate as nitrogen, as required by the KDHE for disposal of investigation-derived waste. 


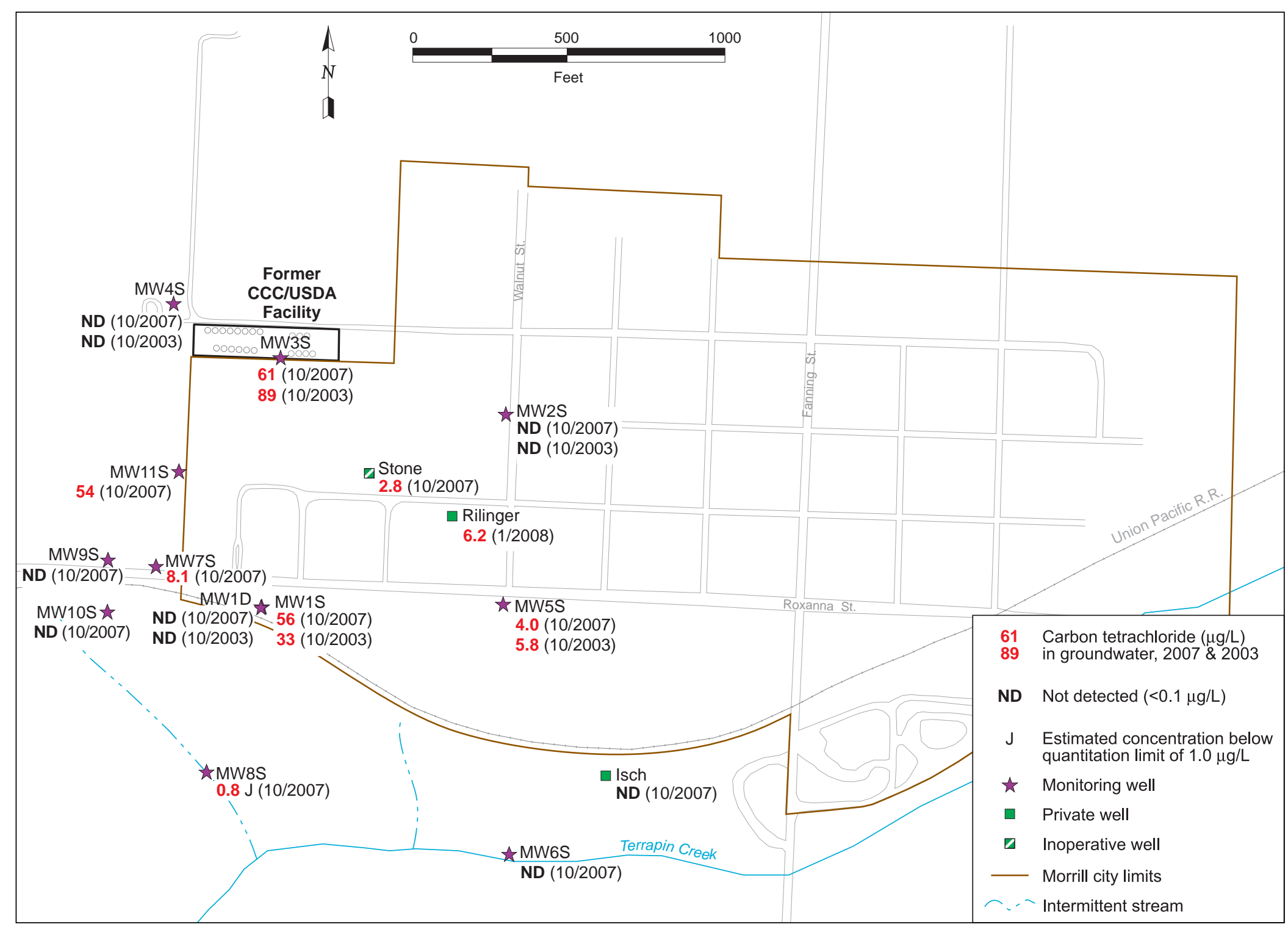

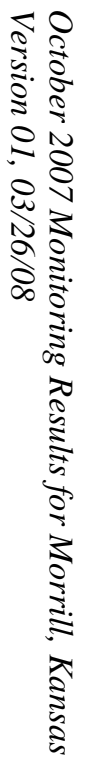

FIGURE 4.1 Carbon tetrachloride levels in groundwater at Morrill in October 2003 and October 2007. 


\section{References}

Argonne, 2002, Final Master Work Plan: Environmental Investigations at Former CCC/USDA Facilities in Kansas, 2002 Revision, ANL/ER/TR-02/2004, prepared for the Commodity Credit Corporation, U.S. Department of Agriculture, by Argonne National Laboratory, Argonne, Illinois, December.

Argonne, 2004, Final Phase I-Phase II Interim Report: Expedited Site Characterization, Morrill, Kansas, ANL/ER/TR-04/001, prepared for the Commodity Credit Corporation, U.S. Department of Agriculture, by Argonne National Laboratory, Argonne, Illinois, December.

Argonne, 2005a, Final Report, Monitoring Well Installation and Sampling, 2004, at Morrill, Kansas, ANL/ER/TR-04/010, prepared for the Commodity Credit Corporation, U.S. Department of Agriculture, by Argonne National Laboratory, Argonne, Illinois, November.

Argonne, 2005b, Final Work Plan: Groundwater Monitoring at Morrill, Kansas, ANL/ER/TR05/003, prepared for the Commodity Credit Corporation, U.S. Department of Agriculture, by Argonne National Laboratory, Argonne, Illinois, August.

Argonne, 2005c, Draft Engineering Evaluation/Cost Analysis for the Murdock Groundwater Contamination Site, Murdock, Nebraska, ANL/ER/TR-05/005, prepared for the Commodity Credit Corporation, U.S. Department of Agriculture, Washington, D.C., by Argonne National Laboratory, Argonne, Illinois, April.

Argonne, 2006a, September 2006 Monitoring Results for Morrill, Kansas, ANL/EVS/AGEM/CHRON-1016, prepared for the Commodity Credit Corporation, U.S. Department of Agriculture, Washington, D.C., by Argonne National Laboratory, Argonne, Illinois, December.

Argonne, 2006b, Final Monitoring Plan for Site Restoration at Murdock, Nebraska, ANL/EVS/AGEM/TR-05-04, prepared for the Commodity Credit Corporation, U.S. Department of Agriculture, Washington, D.C., by Argonne National Laboratory, Argonne, Illinois, February.

Argonne, 2007a, Proposed Addendum to the Groundwater Monitoring Plan for Morrill, Kansas, ANL/EVS/AGEM/CHRON-1042, prepared for the Commodity Credit Corporation, 
U.S. Department of Agriculture, Washington, D.C., by Argonne National Laboratory, Argonne, Illinois, May 3.

Argonne, 2007b, AGEM-15: Standard Operating Procedure for Sediment Sampling, ANL/EVS/AGEM/CHRON-1042, prepared for the Commodity Credit Corporation, U.S. Department of Agriculture, Washington, D.C., by Argonne National Laboratory, Argonne, Illinois, February 21.

Argonne, 2007c, March-April Monitoring Results for Morrill, Kansas, ANL/EVS/AGEM/TR07-06, prepared for the Commodity Credit Corporation, U.S. Department of Agriculture, Washington, D.C., by Argonne National Laboratory, Argonne, Illinois, May.

Argonne, 2007d, Summary of Operations and Performance of the Murdock Site Restoration Project in June 2005-December 2006, ANL/EVS/AGEM/TR-07-05, prepared for the Commodity Credit Corporation, U.S. Department of Agriculture, Washington, D.C., by Argonne National Laboratory, Argonne, Illinois, May.

Argonne, 2007e, Final Report: Groundwater Monitoring at Morrill, Kansas, in September 2005 and March 2006, with Expansion of the Monitoring Network in January 2006, ANL/EVS/AGEM/TR-06-09, prepared for the Commodity Credit Corporation, U.S. Department of Agriculture, Washington, D.C., by Argonne National Laboratory, Argonne, Illinois, June.

Argonne, 2007f, Summary of Operations and Performance of the Murdock Site Restoration Project in June 2005-December 2006, ANL/EVS/AGEM/TR-07-05, prepared for the Commodity Credit Corporation, U.S. Department of Agriculture, Washington, D.C., by Argonne National Laboratory, Argonne, Illinois, May.

GeoCore, 1996, Environmental Site Investigation Report: Phase II Drilling and Sampling: Morrill Public Water Supply Well \#5, Morrill, Kansas, prepared by GeoCore Services, Inc., Salina, Kansas, for the Bureau of Environmental Remediation, Kansas Department of Health and Environment, Topeka, Kansas (draft issued August 31, 1995; revised February 29, 1996). 
EPA, 1995, Method 524.2: Measurement of Purgeable Organic Compounds in Water by Capillary Column Gas Chromatography/Mass Spectrometry, Revision 4.1, edited by J.W. Munch, National Exposure Research Laboratory, Office of Research and Development, U.S. Environmental Protection Agency, Cincinnati, Ohio.

EPA, 1998a, Test Methods for Evaluating Solid Waste: Physical/Chemical Methods, EPA SW846, 3rd edition, Draft Update IVA, U.S. Environmental Protection Agency, January (available from National Technical Information Service, 5285 Port Royal Road, Springfield, VA 22161).

EPA, 1998b, Technical Protocol for Evaluating Natural Attenuation of Chlorinated Solvents in Ground Water, EPA/600/R-98/128, Office of Research and Development, U.S. Environmental Protection Agency, Washington, D.C., September (http://www.epa.gov/superfund/resources/ gwdocs/protocol.htm).

Kampbell, D.H., and S.A. Vandegrift, 1998, "Analysis of Dissolved Methane, Ethane, and Ethylene in Ground Water by a Standard Chromatographic Technique," Journal of Chromatographic Science 36:253-256.

KDHE, 2001, Monitored Natural Attenuation, Policy BER-RS-042, Bureau of Environmental Remediation, Kansas Department of Health and Environment, Topeka, Kansas, March 30 (revised December 18, 2005; http://www.kdheks.gov/ber/policies/BER_RS_042.pdf).

KDHE, 2007a, letter from C. Carey (Bureau of Environmental Remediation, Kansas Department of Health and Environment, Topeka, Kansas) to C. Roe (Commodity Credit Corporation, U.S. Department of Agriculture, Washington, D.C.) regarding Draft Report: Groundwater Monitoring at Morrill, Kansas, in September 2005 and March 2006, with Expansion of the Monitoring Network in January 2006, January 5.

KDHE, 2007b, letter from C. Carey (Bureau of Environmental Remediation, Kansas Department of Health and Environment, Topeka, Kansas) to C. Roe (Commodity Credit Corporation, U.S. Department of Agriculture, Washington, D.C.) regarding March-April 2007 Monitoring Results for Morrill, Kansas, August 1. 
LaFreniere, L.M., M.C. Negri, R.A. Sedivy, Y.E. Yan, D. Steck, S.M. Gilmore, P. Kulakow, S. Hutchinson, and L. Erickson, 2006, "Integrating Phytoremediation, Wetlands, Spray Irrigation, and Prairie Restoration to Treat Carbon Tetrachloride Contamination in a Rural Community," pp.367-388 in Proceedings of the 29th Arctic and Marine Oilspill Program (AMOP) Technical Seminar, June 6-8, 2006, Vancouver, British Columbia, Canada, Environment Canada.

NAIP, 2005, aerial photograph (titled mosaic) of Brown County, Kansas, naip_11_2n_s_ks013_2005_1, National Agriculture Imagery Program, U.S. Department of Agriculture, Salt Lake City, Utah (http://www.apfo.usda.gov/NAIP.html).

Sabetha, 2007, general receipt, Office of City Clerk, City of Sabetha, Kansas, acknowledging receipt of payment from Argonne National Laboratory for purged water, October 25. 


\section{Appendix A:}

Sequence of Sampling Activities at Morrill, Kansas, in October 2007 
TABLE A.1 Sequence of sampling activities at Morrill in October 2007.

\begin{tabular}{|c|c|c|c|c|c|c|c|c|}
\hline $\begin{array}{l}\text { Sample } \\
\text { Date }\end{array}$ & Time & Location & Sample & Medium & Type $^{a}$ & $\begin{array}{l}\text { Depth } \\
\text { (ft TOC) }\end{array}$ & $\begin{array}{l}\text { Chain of } \\
\text { Custody No. }\end{array}$ & Description \\
\hline $10 / 01 / 07$ & $10: 58$ & MW1S & MRMW1S-W-16595 & Water & MW & $11-51$ & 3987 & $\begin{array}{l}\text { Depth to water from top of casing }(\mathrm{TOC})=21.65 \mathrm{ft} \text {. Depth } \\
\text { of well }=53.95 \mathrm{ft} \text {. Sample collected from } 4 \text {-in. well at } \\
\text { low flow after purging of } 63 \text { gal with Redi-Flo pump. } \\
\text { Purged to container. }\end{array}$ \\
\hline $10 / 01 / 07$ & $10: 59$ & MW1S & MRMW1S-W-16595D & Water & MW & $11-51$ & 3987 & Replicate of sample MRMW1S-W-16595. \\
\hline $10 / 01 / 07$ & $11: 10$ & QC & MRQCRI-W-16597 & Water & $\mathrm{RI}$ & - & 3987 & $\begin{array}{l}\text { Rinsate of decontaminated pump and hose after collection } \\
\text { of sample MRMW1S-W- } 16595 \text {. }\end{array}$ \\
\hline $10 / 01 / 07$ & $11: 26$ & MW1D & MRMW1D-W-16596 & Water & MW & $63-88$ & 3987 & $\begin{array}{l}\text { Depth to water from TOC }=22.75 \mathrm{ft} \text {. Depth of well }= \\
89.38 \mathrm{ft} \text {. Sample collected from } 4 \text {-in. well at low flow } \\
\text { after purging of } 130 \text { gal with Redi-Flo pump. Purged to } \\
\text { container. }\end{array}$ \\
\hline $10 / 01 / 07$ & $13: 41$ & MW10S & MRMW10S-W-16593 & Water & MW & $30-45$ & 3987 & $\begin{array}{l}\text { Depth to water from TOC }=6.95 \mathrm{ft} \text {. Depth of well = } \\
49.65 \mathrm{ft} \text {. Sample collected from } 2 \text {-in. well at low flow } \\
\text { after purging of } 20 \mathrm{gal} \text { with Redi-Flo pump. Purged to } \\
\text { container. }\end{array}$ \\
\hline $10 / 01 / 07$ & 15:05 & MW11S & MRMW11S-W-16594 & Water & MW & $53-68$ & 3987 & $\begin{array}{l}\text { Depth to water from TOC }=31.55 \mathrm{ft} \text {. Depth of well }= \\
73.00 \mathrm{ft} \text {. Sample collected from } 2 \text {-in. well at low flow } \\
\text { after purging of } 20 \text { gal with Redi-Flo pump. Purged to } \\
\text { container. }\end{array}$ \\
\hline $10 / 01 / 07$ & 15:06 & MW11S & MRMW11S-W-16594D & Water & MW & $53-68$ & 3987 & Replicate of sample MRMW11S-W-16594. \\
\hline $10 / 01 / 07$ & $15: 54$ & MW7S & MRMW7S-W-16581 & Water & MW & $20-45$ & 3987 & $\begin{array}{l}\text { Depth to water from TOC }=12.40 \mathrm{ft} \text {. Depth of well = } \\
47.00 \mathrm{ft} \text {. Sample collected from } 4 \text {-in. well at low flow } \\
\text { after purging of } 70 \text { gal with Redi-Flo pump. Purged to } \\
\text { container. }\end{array}$ \\
\hline $10 / 01 / 07$ & $16: 30$ & MW9S & MRMW9S-W-16582 & Water & MW & $\begin{array}{l}38.83- \\
53.83\end{array}$ & 3987 & $\begin{array}{l}\text { Depth to water from TOC }=14.00 \mathrm{ft} \text {. Depth of well = } \\
58.60 \mathrm{ft} \text {. Sample collected from } 2 \text {-in. well at low flow } \\
\text { after purging of } 23 \text { gal with Redi-Flo pump. Purged to } \\
\text { container. }\end{array}$ \\
\hline $10 / 02 / 07$ & $7: 46$ & MW6S & MRMW6S-W-16583 & Water & MW & $10-25$ & 3986 & $\begin{array}{l}\text { Depth to water from TOC }=5.00 \mathrm{ft} \text {. Depth of well = } \\
26.90 \mathrm{ft} \text {. Sample collected from } 4 \text {-in. well at low flow } \\
\text { after purging of } 31 \text { gal with Redi-Flo pump. Purged to } \\
\text { container. Well going dry at end of purge. }\end{array}$ \\
\hline $10 / 02 / 07$ & $9: 10$ & MW8S & MRMW8S-W-16584 & Water & MW & $10-25$ & 3986 & $\begin{array}{l}\text { Depth to water from TOC }=2.20 \mathrm{ft} \text {. Depth of well }= \\
26.80 \mathrm{ft} \text {. Sample collected from } 4 \text {-in. well at low flow } \\
\text { after purging of } 48 \mathrm{gal} \text { with Redi-Flo pump. Purged to } \\
\text { container. }\end{array}$ \\
\hline
\end{tabular}


TABLE A.1 (Cont.)

\begin{tabular}{|c|c|c|c|c|c|c|c|c|}
\hline $\begin{array}{c}\text { Sample } \\
\text { Date }\end{array}$ & Time & Location & Sample & Medium & Type ${ }^{a}$ & $\begin{array}{l}\text { Depth } \\
\text { (ft TOC) }\end{array}$ & $\begin{array}{c}\text { Chain of } \\
\text { Custody No. }\end{array}$ & Description \\
\hline $10 / 02 / 07$ & $12: 12$ & QC & MRQCFB-W-16599 & Water & $\mathrm{FB}$ & - & 3986 & $\begin{array}{l}\text { Field blank of water used during October } 2007 \text { sampling } \\
\text { event. }\end{array}$ \\
\hline $10 / 02 / 07$ & $12: 22$ & QC & MRQCTB-W-16598 & Water & TB & - & 3986 & $\begin{array}{l}\text { Trip blank sent to the AGEM Laboratory for organic } \\
\text { analyses with water samples listed on chain-of-custody } \\
\text { forms (COCs) } 3987 \text { and } 3986 \text {. }\end{array}$ \\
\hline $10 / 02 / 07$ & $12: 23$ & QC & MRQCTB-W-16598A & Water & TB & - & 6276 & $\begin{array}{l}\text { Trip blank sent to Envirosystems, Inc., for verification } \\
\text { organic analysis with water samples listed on COC } \\
6276 .\end{array}$ \\
\hline $10 / 02 / 07$ & $12: 24$ & QC & MRQCTB-W-16599 & Water & TB & - & 3992 & $\begin{array}{l}\text { Trip blank sent to Severn-Trent Laboratories for methane } \\
\text { analysis with water samples listed on COCs } 3990 \text { and } \\
3992 \text {. }\end{array}$ \\
\hline $10 / 03 / 07$ & $8: 30$ & MW3S & MRMW3S-W-16585 & Water & MW & $18-48$ & 3984 & $\begin{array}{l}\text { Depth to water from TOC }=22.70 \mathrm{ft} \text {. Depth of well }= \\
47.90 \mathrm{ft} \text {. Sample collected from } 4 \text {-in. well at low flow } \\
\text { after purging of } 50 \text { gal with Redi-Flo pump. Purged to } \\
\text { container. }\end{array}$ \\
\hline $10 / 03 / 07$ & $8: 31$ & MW4S & MRMW4S-W-16586 & Water & MW & $17-47$ & 3984 & $\begin{array}{l}\text { Depth to water from TOC }=31.11 \mathrm{ft} \text {. Depth of well }= \\
47.73 \mathrm{ft} \text {. Sample collected from } 4 \text {-in. well at low flow } \\
\text { after purging of } 30 \text { gal with Redi-Flo pump. Purged to } \\
\text { container. }\end{array}$ \\
\hline $10 / 03 / 07$ & $11: 38$ & MW2S & MRMW2S-W-16587 & Water & MW & $13-53$ & 3984 & $\begin{array}{l}\text { Depth to water from TOC }=31.17 \mathrm{ft} \text {. Depth of well }= \\
53.40 \mathrm{ft} \text {. Sample collected from } 4 \text {-in. well at low flow } \\
\text { after purging of } 44 \mathrm{gal} \text { with Redi-Flo pump. Purged to } \\
\text { container. }\end{array}$ \\
\hline $10 / 03 / 07$ & $11: 47$ & MW5S & MRMW5S-W-16588 & Water & MW & $15-55$ & 3984 & $\begin{array}{l}\text { Depth to water from TOC }=19.55 \mathrm{ft} \text {. Depth of well }= \\
54.65 \mathrm{ft} \text {. Sample collected from } 4 \text {-in. well at low flow } \\
\text { after purging of } 68 \text { gal with Redi-Flo pump. Purged to } \\
\text { container. }\end{array}$ \\
\hline $10 / 03 / 07$ & $13: 04$ & Rilinger & MRRILINGER-W-16591 & Water & DW & - & 3984 & $\begin{array}{l}\text { Wiring to pump had been repaired. Allowed pump to run } \\
\text { for several minutes, and then collected samples. }\end{array}$ \\
\hline $10 / 03 / 07$ & $13: 22$ & Isch & MRISCH-W-16590 & Water & DW & - & 3984 & Water was running continuously; collected samples. \\
\hline $10 / 03 / 07$ & $13: 30$ & QC & MRQCTB-W-16604 & Water & TB & - & 6278 & $\begin{array}{l}\text { Trip blank sent to Severn-Trent Laboratories for methane } \\
\text { analysis with water samples listed on COC } 6278 \text {. }\end{array}$ \\
\hline $10 / 03 / 07$ & 14:09 & Stone & MRSTONE-W-16589 & Water & DW & - & 3984 & $\begin{array}{l}\text { Depth to water from TOC }=14.60 \mathrm{ft} \text {. Depth of well }= \\
38.60 \mathrm{ft} \text {. Sample collected from } 4 \text {-in. well at low flow } \\
\text { after purging of } 72 \mathrm{gal} \text { with Redi-Flo pump. Purged to } \\
\text { container. }\end{array}$ \\
\hline
\end{tabular}


TABLE A.1 (Cont.)

\begin{tabular}{|c|c|c|c|c|c|c|c|c|}
\hline $\begin{array}{l}\text { Sample } \\
\text { Date }\end{array}$ & Time & Location & Sample & Medium & Type $^{a}$ & $\begin{array}{c}\text { Depth } \\
\text { (ft TOC) }\end{array}$ & $\begin{array}{c}\text { Chain of } \\
\text { Custody No. }\end{array}$ & Description \\
\hline $10 / 03 / 07$ & $14: 15$ & QC & MRQCTB-W-16602 & Water & TB & - & 3984 & $\begin{array}{l}\text { Trip blank sent to the AGEM Laboratory for organic } \\
\text { analyses with water samples listed on COC } 3984 \text {. }\end{array}$ \\
\hline $10 / 08 / 07$ & $10: 13$ & SMB & MRSMB-W-16581 & Water & SW & - & 6279 & Water sample from Terrapin Creek. \\
\hline $10 / 08 / 07$ & $10: 14$ & SMB & MRSMB-S-16582 & Soil & Sed & - & 6280 & Sediment sample from Terrapin Creek. \\
\hline $10 / 08 / 07$ & $10: 29$ & SM1 & MRSM1-W-16583 & Water & SW & - & 6279 & Water sample from Terrapin Creek. \\
\hline $10 / 08 / 07$ & $10: 30$ & SM1 & MRSM1-S-16584 & Soil & Sed & - & 6280 & Sediment sample from Terrapin Creek. \\
\hline $10 / 08 / 07$ & $10: 44$ & SM2 & MRSM2-W-16585 & Water & SW & - & 6279 & Water sample from Terrapin Creek. \\
\hline $10 / 08 / 07$ & $10: 45$ & SM2 & MRSM2-S-16586 & Soil & Sed & - & 6280 & Sediment sample from Terrapin Creek. \\
\hline $10 / 08 / 07$ & $10: 55$ & SM3 & MRSM3-W-16587 & Water & SW & - & 6279 & Water sample from Terrapin Creek. \\
\hline $10 / 08 / 07$ & $10: 56$ & SM3 & MRSM3-S-16588 & Soil & Sed & - & 6280 & Sediment sample from Terrapin Creek. \\
\hline $10 / 08 / 07$ & $11: 05$ & SM4 & MRSM4-W-16589 & Water & SW & - & 6279 & Water sample from Terrapin Creek. \\
\hline $10 / 08 / 07$ & $11: 06$ & SM4 & MRSM4-S-16590 & Soil & Sed & - & 6280 & Sediment sample from Terrapin Creek. \\
\hline $10 / 08 / 07$ & $11: 10$ & QC & MRQCTB-W-16591 & Water & TB & - & 6279 & $\begin{array}{l}\text { Trip blank sent to the AGEM Laboratory for organic } \\
\text { analyses with water samples listed on COC } 6279 \text {. }\end{array}$ \\
\hline $10 / 08 / 07$ & $11: 11$ & QC & MRQCTB-S-16592 & Soil & TB & - & 6280 & $\begin{array}{l}\text { Trip blank sent to the AGEM Laboratory for organic } \\
\text { analyses with soil samples listed on COC } 6280 .\end{array}$ \\
\hline $10 / 08 / 07$ & $11: 25$ & Rilinger & MRRILINGER-W-16592 & Water & DW & - & 6279 & $\begin{array}{l}\text { Resampling of Rilinger well. Allowed pump run for } 5 \mathrm{~min} \text {, } \\
\text { and then sampled. }\end{array}$ \\
\hline $10 / 09 / 07$ & $7: 10$ & QC & MR-WW & Water & BT & - & 1132596 & $\begin{array}{l}\text { Containerized waste purge water generated during } \\
\text { October } 2007 \text { monitoring event. Analyzed by Pace } \\
\text { Analytical Services for VOCs, ethylene dibromide, and } \\
\text { nitrate. }\end{array}$ \\
\hline $1 / 11 / 08$ & $14: 11$ & MW4S & MOMW4S-W-011108 & Water & MW & $17-47$ & 6025 & $\begin{array}{l}\text { Resampling of upgradient monitoring well to confirm that } \\
\text { trace carbon tetrachloride detection of } 10 / 3 / 07 \text { was a } \\
\text { result of incomplete equipment decontamination. }\end{array}$ \\
\hline $1 / 11 / 08$ & $14: 50$ & Rilinger & MORIL-W-11108 & Water & DW & - & 6025 & $\begin{array}{l}\text { Resampling of Rilinger well. Sample collected after outside } \\
\text { tap had run for } 10 \text { min. }\end{array}$ \\
\hline $1 / 14 / 08$ & $17: 06$ & QC & UTQCTB-W-24382 & Water & TB & - & 6025 & $\begin{array}{l}\text { Trip blank sent to the AGEM Laboratory for organic } \\
\text { analyses with water samples listed on COC } 6025 \text {. }\end{array}$ \\
\hline
\end{tabular}

a Sample types: BT, wastewater composite; DW, domestic well; FB, field blank; MW, monitoring well; RI, rinsate; Sed, sediment; SW, surface water; TB, trip blank. 


\section{Appendix B:}

Data Summary for Verification VOCs Analyses by Envirosystems, Inc. 


\section{ENVIROSYSTEMS, INC.}

9200 Rumsey Road - Suite B102 - Columbia, Maryland 21045-1934

Phone (410) 964-0330 • Fax (410) 740-9306

Email: info@envsystems.com•Webpage: www.envsystems.com/envsys

October 10, 2007

Jorge S. Alvarado, PH. D

Argonne National Laboratory

Environmental Research Division

Applied Geosciences and Environmental

Management Section

9700 South Cass Avenue, ER-203

Argonne, Illinois 60439

RE: Report \#070288

Dear Jorge,

Enclosed is the Analytical Data Package for Organics Analysis for the samples received on October 03,2007 . These samples were analyzed by using method SW-846 8260B and USEPA CLP SOWOLM04.3 and the chain of custody instructions.

Please do not hesitate to call if you have any questions, comments, or require additional information.

Sincerely,

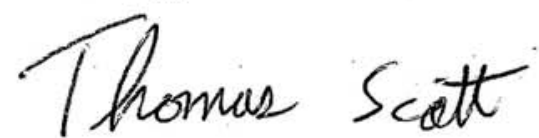

Mohan Khare Ph.D.

President/CEO

Enclosure (1)

$\mathrm{MK} / \mathrm{ncc}$ 


\section{SDG NARRATIVE \\ VOLATILE ORGANICS (VOC)}

Envirosystems, Inc.

Contract: N/A

Client: Argonne National Laboratory

Case: N/A

SDG: ARG71002

\section{SAMPLE RECIEPT}

Date received: $10-03-2007$

Cooler Temperature: 2

\section{Sample Summary}

\begin{tabular}{|c|c|c|c|}
\hline Client ID & Laboratory ID & Matrix & pH \\
\hline MR-MWIS-W-16595 & $0071002-01$ & WATER & 7 \\
\hline MR-MWIIS-W-16594 & $0071002-02$ & WATER & 7 \\
\hline MR-QCTB-W-16598 & $0071002-03$ & WATER & 7 \\
\hline
\end{tabular}

\section{HOLDING TIMES}
A. Sample Preparation: All holding times were met.

B. Sample Analysis: All holding times were met

\section{METHODS}

The samples were analyzed and reported by using method SW-846 $8260 \mathrm{~B}$ and USEPA CLP SOW OLM04.3 for target compound list.

\section{INSTRUMENT AND CHROMATOGRAPHIC CONDITIONS}

A Hewlett Packard 6890 gas chromatograph equipped with a Hewlett Packard 5975 MSD was used for sample analysis. The capillary column used was a Restek $20 \mathrm{~m}$ by $0.18 \mathrm{~mm}$ ID by $1.0 \mu \mathrm{m}$ film thickness (Restek Cat. \# RTX-624). The trap used with the sample concentrator is an OI Analytical Trap \#10, $30 \mathrm{~cm}$ packed with Tenax/silica gel/cms (PN\#228122).

\section{PREPARATION}

The submitted samples were analyzed as received.

6. ANALYSIS

A. Calibration:

I. Initial calibration

All acceptance criteria as stipulated by SW- 8468260 b were met for all SPCC's and CCC's. All target compounds met the required percent RSD. 


\section{SDG NARRATIVE VOLATILE ORGANICS (VOC)}

\section{Blanks:}

All acceptance criteria were met.

\section{Surrogates:}

All acceptance criteria were met.

B. Spikes:

\section{Laboratory Control Spikes (LCS)}

LCS and LCSD samples were not analyzed.

\section{Matrix Spike/Matrix Spike Duplicate (MS/MSD)}

The client did not request a MS/MSD.

\section{Internal Standards:}

All acceptance criteria were met.

\section{Samples}

Sample analysis proceeded normally.

I certify that this Sample Data Package is in compliance with the terms and conditions of the contract, both technically and for completeness, for other than the conditions detailed above. Release of the data contained in the hard copy Sample Data Package and in the Electronic Data Deliverables has been authorized by the laboratory manager or the manager's designee, as verified by the following signatures.

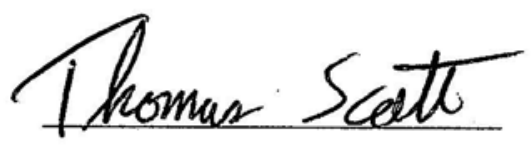

Laboratory Manager

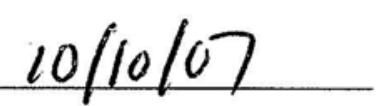

Date 


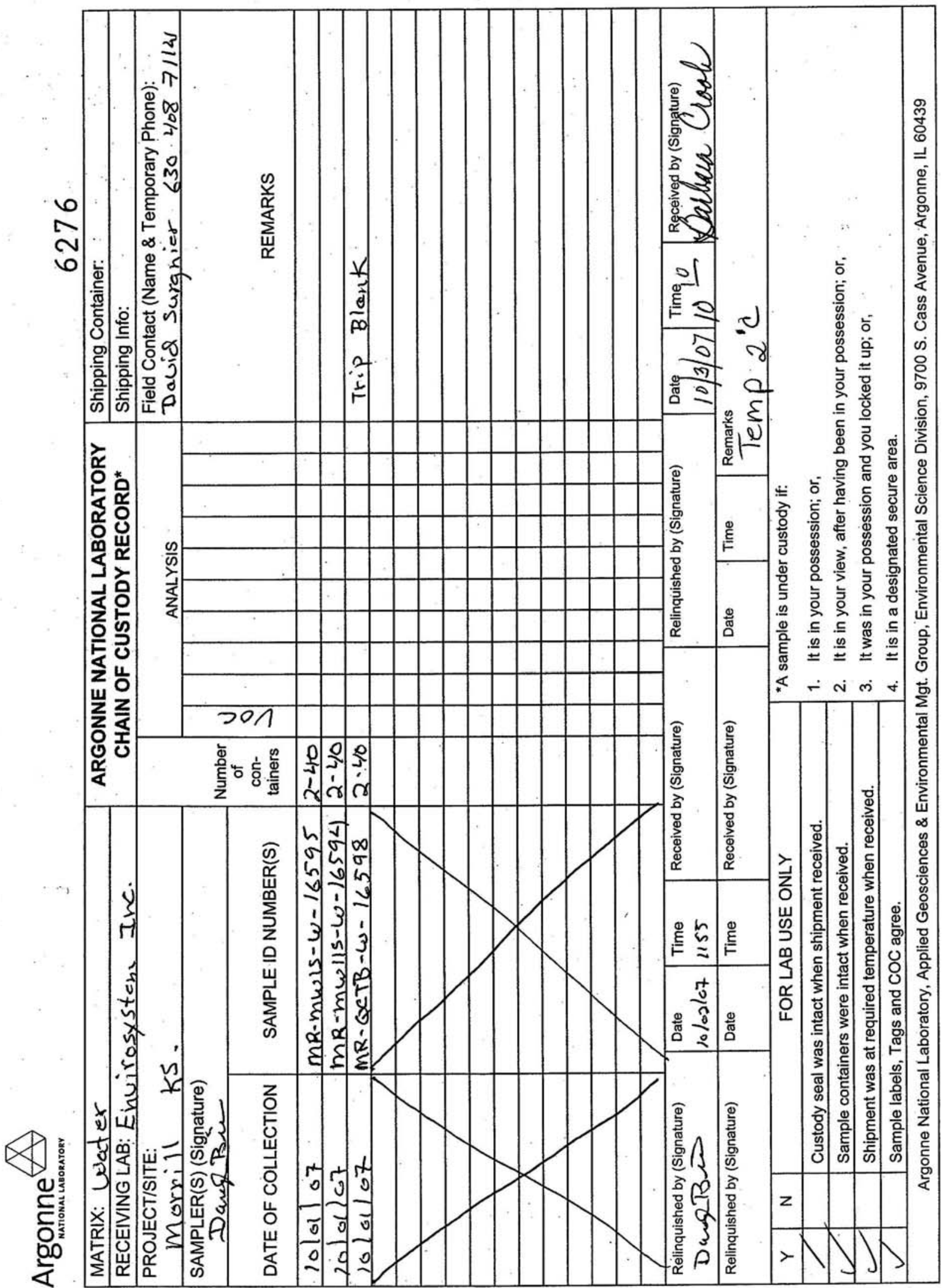


$1 A$ - FORM I VOA-1

VOLATILE ORGANICS ANALYSIS DATA SHEET
EPA SAMPLE NO.

MR-MWIS-W-16595

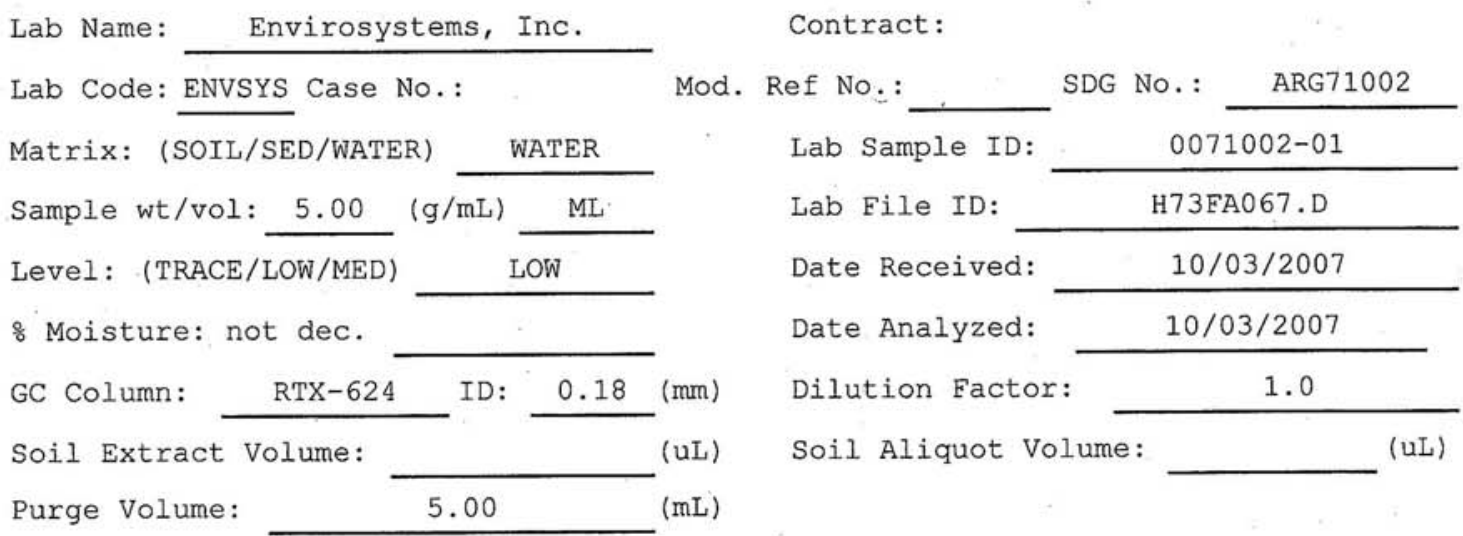

\begin{tabular}{|c|c|c|c|}
\hline CAS NO. & COMPOUND & $\begin{array}{l}\text { CONCENTRATION UNITS: } \\
(\mathrm{ug} / \mathrm{L} \text { or } \mathrm{ug} / \mathrm{kg}) \mathrm{UG} / \mathrm{L}\end{array}$ & Q \\
\hline $75-71-8$ & Dichlorodifluoromethane & 5.0 & $\mathrm{U}$ \\
\hline $74-87-3$ & Chloromethane & 5.0 & $\mathrm{U}$ \\
\hline $75-01-4$ & Vinyl chloride & 5.0 & $\mathrm{U}$ \\
\hline $74-83-9$ & Bromomethane & 5.0 & $\mathrm{U}$ \\
\hline $75-00-3$ & Chloroethane & 5.0 & $\mathrm{U}$ \\
\hline $75-69-4$ & Trichlorofluoromethane & 5.0 & $\mathrm{U}$ \\
\hline $75-35-4$ & 1,1-Dichloroethene & 5.0 & $\mathrm{U}$ \\
\hline $76-13-1$ & 1,1,2-Trichloro-1,2,2-trifluoroethane & 5.0 & $\mathrm{U}$ \\
\hline $67-64-1$ & Acetone & 5.0 & $\mathrm{U}$ \\
\hline $75-15-0$ & Carbon disulfide & 5.0 & 0 \\
\hline $79-20-9$ & Methyl acetate & 5.0 & $\mathrm{U}$ \\
\hline $75-09-2$ & Methylene chloride & 5.0 & $\cdot 0$ \\
\hline $156-60-5$ & trans-1,2-Dichloroethene & 5.0 & 0 \\
\hline $1634-04-4$ & Methyl tert-butyl ether & 5.0 & $\mathrm{U}$ \\
\hline $75-34-3$ & 1,1-Dichloroethane & 5.0 & $\mathrm{U}$ \\
\hline $156-59-2$ & cis-1,2-Dichloroethene & 5.0 & $\mathrm{U}$ \\
\hline $78-93-3$ & 2-Butanone & 5.0 & $\mathrm{U}$ \\
\hline $67-66-3$ & Chloroform & 2.5 & $\mathrm{~J}$ \\
\hline $71-55-6$ & 1,1,1-Trichloroethane & 5.0 & $\mathrm{U}$ \\
\hline $110-82-7$ & Cyclohexane & 5.0 & $\mathrm{U}$ \\
\hline $56-23-5$ & Carbon Tetrachloride & 32 & \\
\hline $71-43-2$ & Benzene & 5.0 & 0 \\
\hline $107-06-2$ & 1,2-Dichloroethane & 5.0 & $\mathrm{U}$ \\
\hline
\end{tabular}


$1 B$ - FORM I VOA-2

VOLATILE ORGANICS ANALYSIS DATA SHEET
EPA SAMPLE NO.

MR-MWIS-W-16595

\begin{tabular}{|c|c|c|c|c|c|c|c|}
\hline Lab Name: & Envirosystems, & & & Contract: & & & \\
\hline Lab Code: ENI & VSYS Case No.: & & Mod. & Ref No.: & SDG & G No.: & 1002 \\
\hline Matrix: (SOI & L/SED/WATER) & TER & & Lab Sampl & le ID: & $0071002-$ & \\
\hline Sample wt/vo & $1: 5.00 \quad(\mathrm{~g} / \mathrm{mL})$ & ML & & Lab File & ID: & H73FA067. & \\
\hline Level: (TRAC & E/LOW/MED) & & & Date Rece & eived: & $10 / 03 / 20$ & \\
\hline \& Moisture: & not dec. & & & Date Anal & 1yzed: & $10 / 03 / 20$ & \\
\hline GC Column: & RTX-624 ID: & 0.18 & $(\mathrm{~mm})$ & Dilution & Factor: & 1.0 & . \\
\hline Soil Extract & Volume: & & (UL) & Soil Aliq & quot Volum & me: & (uL) \\
\hline Purge Volume & 5.00 & & $(\mathrm{~mL})$ & & & & \\
\hline CAS NO. & COMPOUND & & & & $\begin{array}{l}\text { CONCENTRAT } \\
(\mathrm{ug} / \mathrm{L} \text { or } \mathrm{u}\end{array}$ & $\begin{array}{l}\text { TION UNITS: } \\
\mathrm{ug} / \mathrm{kg}) \mathrm{UG} / \mathrm{L}\end{array}$ & Q \\
\hline $79-01-6$ & Trichloroethene & & & & & 5.0 & $\mathrm{U}$ \\
\hline $108-87-2$ & Methylcyclohexar & & & & & 5.0 & $\mathrm{U}$ \\
\hline $78-87-5$ & 1,2-Dichloroprop & ane & & & & 5.0 & $\mathrm{U}$ \\
\hline $75-27-4$ & Bromodichloromet & rane & & & & 5.0 & $\mathrm{U}$ \\
\hline $10061-01-5$ & cis-1,3-Dichlorc & ropene & & & & 5.0 & $\mathrm{U}$ \\
\hline $108-10-1$ & 4-Methyl-2-penta & cone & & & & 5.0 & $\mathrm{U}$ \\
\hline $108-88-3$ & Toluene & & & & & 5.0 & $\mathrm{U}$ \\
\hline $10061-02-6$ & trans-1,3-Dichlo & coprope & & & & 5.0 & $\mathrm{U}$ \\
\hline $79-00-5$ & 1,1,2-Trichloroe & hane & & & & 5.0 & $\mathrm{U}$ \\
\hline $127-18-4$ & Tetrachloroether & & & & & 5.0 & $\mathrm{U}$ \\
\hline $591-78-6$ & 2-Hexanone & & & & & 5.0 & $\mathrm{U}$ \\
\hline $124-48-1$ & Dibromochloromet & hane & & & & 5.0 & $\mathrm{U}$ \\
\hline $106-93-4$ & 1,2-Dibromoethar & & & & & 5.0 & $\mathrm{U}$ \\
\hline $108-90-7$ & Chlorobenzene & & & & & 5.0 & $\mathrm{U}$ \\
\hline $100-41-4$ & Ethylbenzene & & & & & 5.0 & $\mathrm{U}$ \\
\hline $95-47-6$ & o-Xylene & & & & . & 5.0 & $\mathrm{U}$ \\
\hline $179601-23-1$ & $\mathrm{~m}, \mathrm{p}$-Xylene & & & & & 5.0 & $\mathrm{U}$ \\
\hline $100-42-5$ & Styrene & & & & & 5.0 & $\mathrm{U}$ \\
\hline $75-25-2$ & Bromoform & & & & & 5.0 & $\mathrm{U}$ \\
\hline $98-82-8$ & Isopropylbenzene & & & & & 5.0 & $\mathrm{U}$ \\
\hline $79-34-5$ & $1,1,2,2$-Tetrachl & oroethe & ine & & & 5.0 & $\mathrm{U}$ \\
\hline $541-73-1$ & 1,3-Dichlorobenz & ene & & & & 5.0 & $\mathrm{U}$ \\
\hline $106-46-7$ & 1,4-Dichlorobenz & ene & & & & 5.0 & $\mathrm{U}$ \\
\hline $95-50-1$ & 1,2-Dichlorobenz & ene & & & & 5.0 & $\mathrm{U}$ \\
\hline $96-12-8$ & 1,2-Dibromo-3-ch & loropr & pane & & & 5.0 & $\mathrm{U}$ \\
\hline $120-82-1$ & 1,2,4-Trichlorob & enzene & & & & 5.0 & $\mathrm{U}$ \\
\hline $91-20-3$ & Naphthalene & & & & & 5.0 & $\mathrm{U}$ \\
\hline
\end{tabular}


$1 A$ - FORM I VOA-1

VOLATILE ORGANICS ANALYSIS DATA SHEET
EPA SAMPLE NO.

MR-MWIIS-W-16594

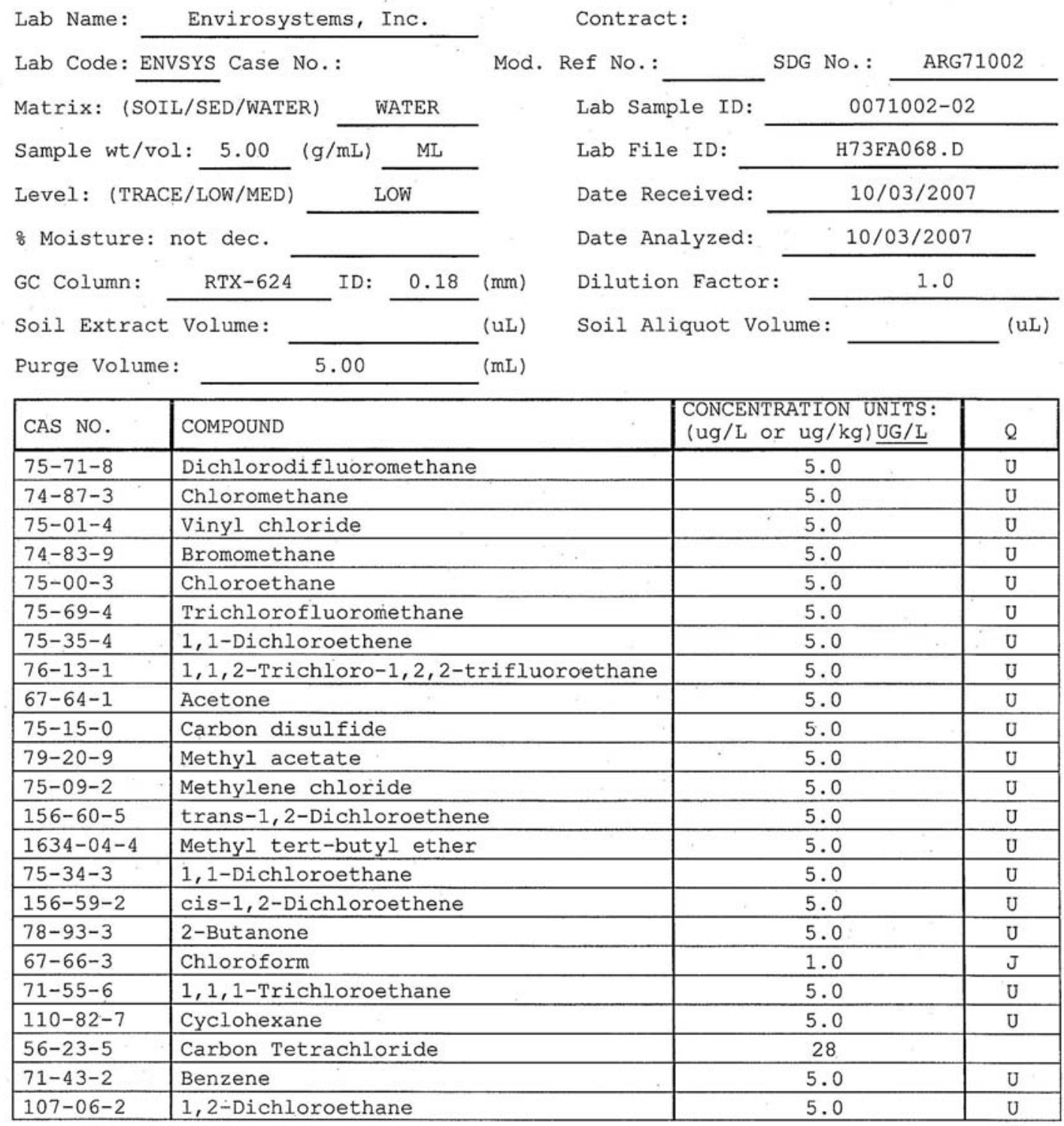


$1 B$ - FORM I VOA-2

VOLATILE ORGANICS ANALYSIS DATA SHEET
EPA SAMPLE NO.

MR-MWI IS-W-16594

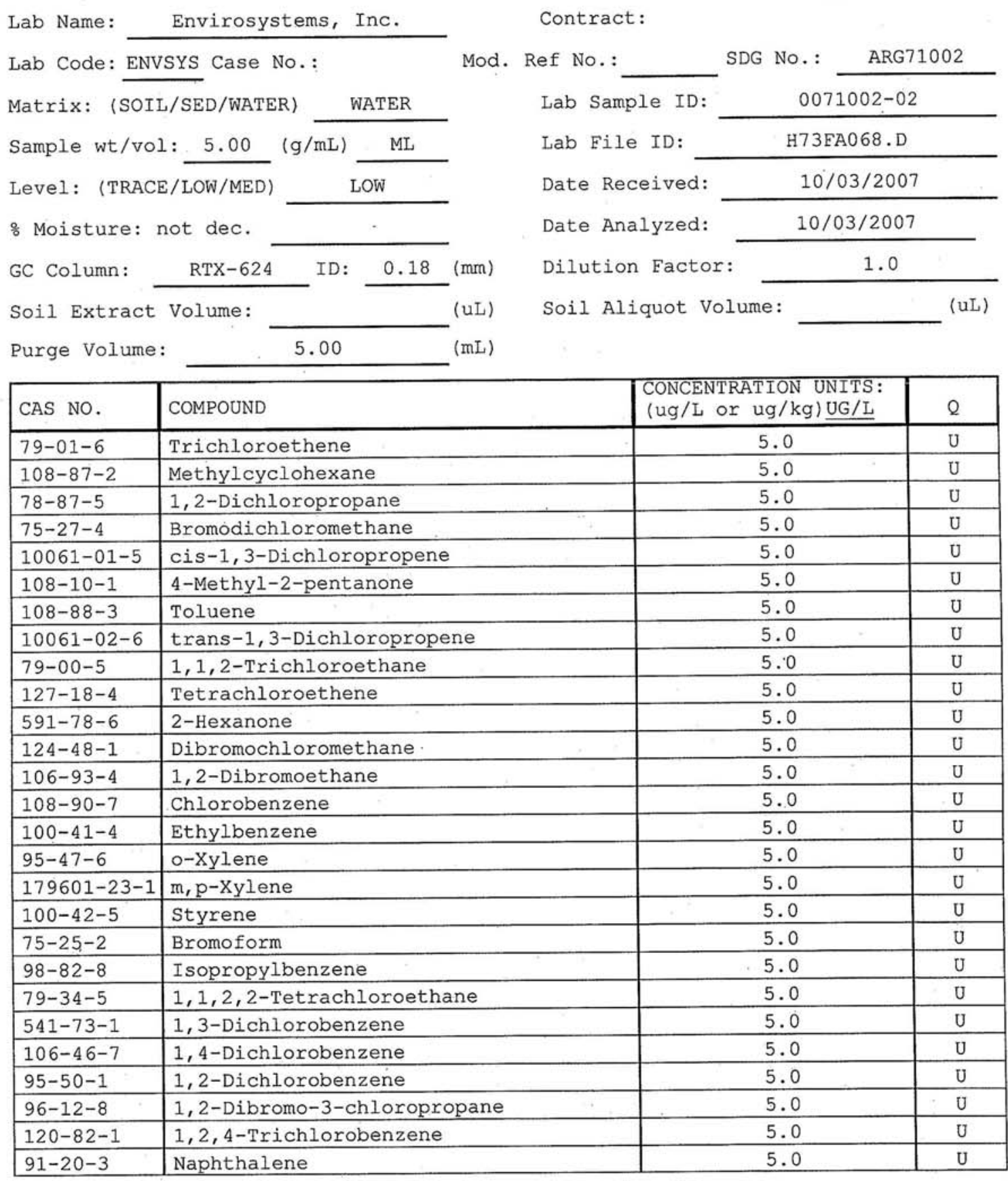


$1 A$ - FORM I VOA-1

VOLATILE ORGANICS ANALYSIS DATA SHEET
EPA SAMPLE NO.

MR-QCTB-W-16598

Lab Name: Envirosystems, Inc.

Contract:

Lab Code: ENVSYS Case No.: Mod. Ref No.: SDG No.: $\quad$ ARG71002

Matrix: (SOIL/SED/WATER) WATER

Sample wt/vol: $\frac{5.00}{\text { Level: (TRACE/LOW/MED) }} \frac{(\mathrm{g} / \mathrm{mL})}{\text { LOW }}$

\& Moisture: not dec.

GC Column: $\quad$ RTX-624 ID: 0.18 (mm)

Soil Extract Volume:

(uL)

Lab Sample ID:

0071002-03

Lab File ID:

H73FA069. D

Date Received:

$10 / 03 / 2007$

Date Analyzed:

$10 / 03 / 2007$

Dilution Factor:

1.0

Soil Aliquot Volume:

(uL)

Purge Volume:

5.00

(mL)

\begin{tabular}{|c|c|c|c|}
\hline CAS NO. & COMPOUND & $\begin{array}{l}\text { CONCENTRATION UNITS: } \\
(\mathrm{ug} / \mathrm{L} \text { or } \mathrm{ug} / \mathrm{kg}) \mathrm{UG} / \mathrm{L}\end{array}$ & Q \\
\hline $75-71-8$ & Dichlorodifluoromethane & 5.0 & $\mathrm{U}$ \\
\hline $74-87-3$ & Chloromethane & 5.0 & $\mathrm{U}$ \\
\hline $75-01-4$ & Vinyl chloride & 5.0 & $\mathrm{U}$ \\
\hline $74-83-9$ & Bromomethane & 5.0 & $\mathrm{U}$ \\
\hline $75-00-3$ & Chloroethane & 5.0 & $\mathrm{U}$ \\
\hline $75-69-4$ & Trichlorofluoromethane & 5.0 & $\mathrm{U}$ \\
\hline $75-35-4$ & 1,1-Dichloroethene & 5.0 & $\mathrm{U}$ \\
\hline $76-13-1$ & 1,1,2-Trichloro-1,2,2-trifluoroethane & 5.0 & $\mathrm{U}$ \\
\hline $67-64-1$ & Acetone & 5.0 & $\mathrm{U}$ \\
\hline $75-15-0$ & Carbon disulfide & 5.0 & $\mathrm{U}$ \\
\hline $79-20-9$ & Methyl acetate & 5.0 & $\mathrm{U}$ \\
\hline $75-09-2$ & Methylene chloride & 5.0 & $\mathrm{U}$ \\
\hline $156-60-5$ & trans-1,2-Dichloroethene & 5.0 & $\mathrm{U}$ \\
\hline $1634-04-4$ & Methyl tert-butyl ether & 5.0 & $\mathrm{U}$ \\
\hline $75-34-3$ & 1,1-Dichloroethane & 5.0 & $\mathrm{U}$ \\
\hline $156-59-2$ & cis-1,2-Dichloroethene & 5.0 & $\mathrm{U}$ \\
\hline $78-93-3$ & 2-Butanone & 5.0 & $\mathrm{U}$ \\
\hline $67-66-3$ & Chloroform & 5.0 & $\mathrm{U}$ \\
\hline $71-55-6$ & 1,1,1-Trichloroethane & 5.0 & $\mathrm{U}$ \\
\hline $110-82-7$ & Cyclohexane & 5.0 & $\mathrm{U}$ \\
\hline $56-23-5$ & Carbon Tetrachloride & 5.0 & $\mathrm{U}$ \\
\hline $71-43-2$ & Benzene & 5.0 & $\mathrm{U}$ \\
\hline $107-06-2$ & 1,2-Dichloroethane & 5.0 & $\mathrm{U}$ \\
\hline
\end{tabular}

SOM01.1 (5/2005) 
$1 B$ - FORM I VOA-2

VOLATILE ORGANICS ANALYSIS DATA SHEET
EPA SAMPLE NO.

MR-QCTB-W-16598

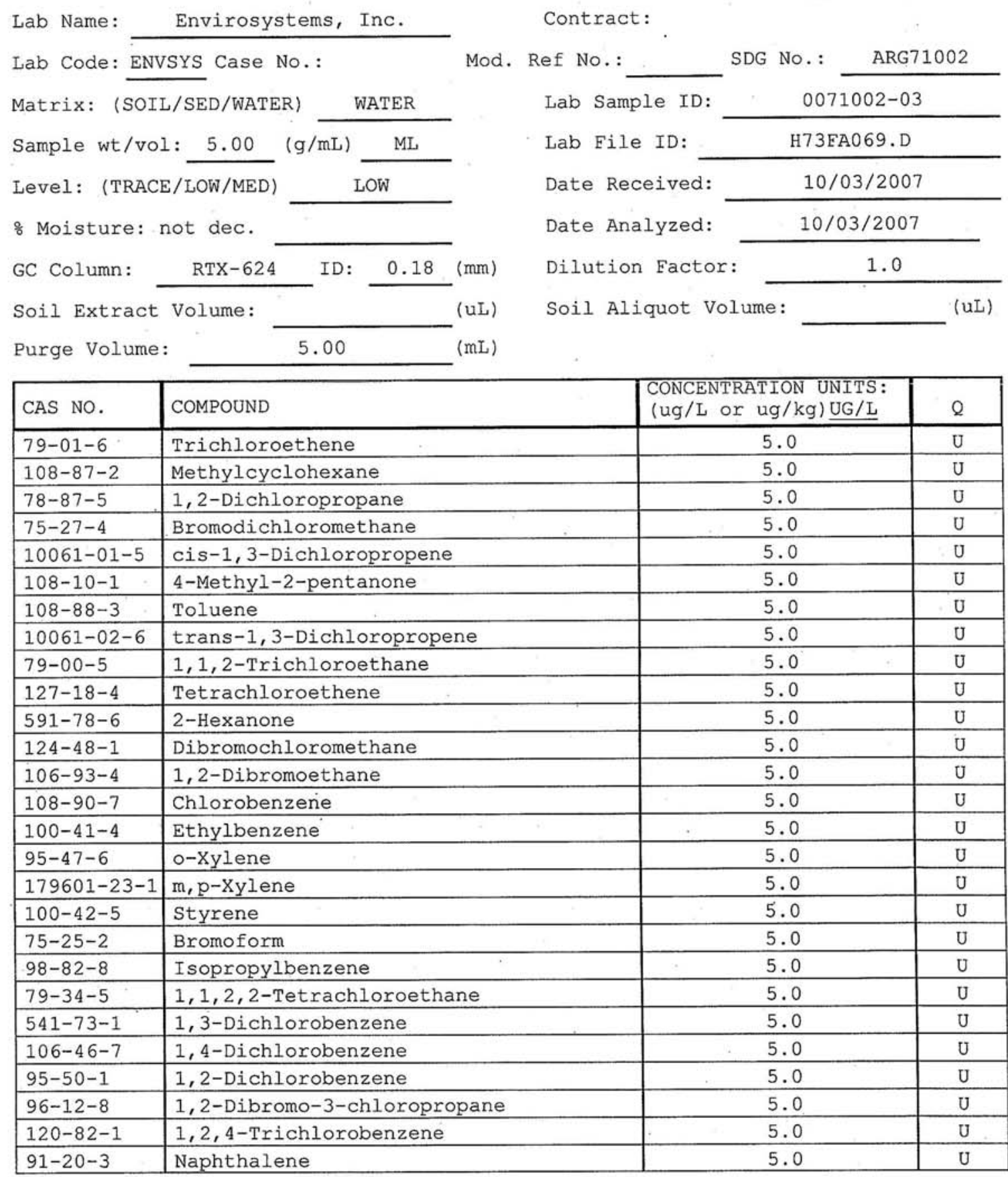


Argonne

Environmental Science Division

Argonne National Laboratory

9700 South Cass Avenue, Bldg. 203

Argonne, IL 60439-4843

www.anl.gov

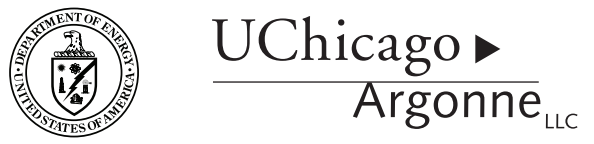

A U.S. Department of Energy laboratory

managed by UChicago Argonne, LLC 